\title{
Psychophysical Evidence for Separate Channels for the Perception of Form, Color, Movement, and Depth
}

\author{
Margaret S. Livingstone and David $\mathbf{H}$. Hubel \\ Department of Neurobiology, Harvard Medical School, Boston, Massachusetts 02115
}

\begin{abstract}
Physiological and anatomical findings in the primate visual system, as well as clinical evidence in humans, suggest that different components of visual information processing are segregated into largely independent parallel pathways. Such a segregation leads to certain predictions about human vision. In this paper we describe psychophysical experiments on the interactions of color, form, depth, and movement in human perception, and we attempt to correlate these aspects of visual perception with the different subdivisions of the visual system.
\end{abstract}

It is function that breathes life into anatomy and perception that vivifies sensory electro-physiology. We must know the logic structure of psycho-physical phenomena if meaning is to be read into electron records from the retina, living or dead. Perhaps we may more surely pick a path through the ever thickening forest of fact if we hold some chart of the pattern of our perceptions.

-Rushton, 1962

Introspection suggests that visual perception can be subdivided into several subprocesses. If asked to list these, most people would include form, color, movement, depth, and perhaps texture. The intuitive impression that vision is multipartite, that it comprises several systems, has been supported by centuries of human psychophysics and by some recent anatomical and physiological studies in primates. In this paper we will try to correlate a large number of observations on human visual perception with these anatomical and physiological findings. To some extent the correlations must be conjectural, not only because our information on the anatomy and physiology of monkeys is far from complete, but also because of the extrapolations

\footnotetext{
Received Aug. 13, 1986; revised Feb. 18, 1987; accepted Apr. 10, 1987.

All the psychophysical observations were made using ourselves as subjects and, for most of the other experiments, other members of our department self-selected by willingness and curiosity. In most tests they were ignorant as to the implications of the experiment. We are grateful to all of them for their patience and enthusiasm. The photography was done by Richard McDonald and Joseph Gagliardi. We thank A. Damasio and H. Damasio for arranging for us to test two of their patients This work was supported by Office of Naval Research Young Investigator Award N00014-86K; office of naval Research Grant N00014-85K; NIH Grants EY00605 and NS000972; a Presidential Young Investigator Award from the NSF, 8352150 and funds from The Monsanto Corporation and from E.I. DuPont de Nemours and $\mathrm{Co}$.

Correspondence should be addressed to Margaret S. Livingstone, Department of Neurobiology, Harvard Medical School, 25 Shattuck St., Boston, MA 02115. Copyright (C) 1987 Society for Neuroscience $0270-6474 / 87 / 113416-53 \$ 02.00 / 0$
}

necessary for the interpretation of human psychophysics in terms of the anatomy and physiology of other primates.

The idea that different aspects of information emanating from common sensory receptors can be processed in parallel along separate pathways in the brain is far from new. Evidence that the mammalian visual system does consist of at least two separate and parallel subpathways can be traced back to the observation that the retinas project both to the lateral geniculate bodies and from there to the cortex and the superior colliculi. The geniculocortical part of the visual pathway is itself subdivided into separate parallel pathways: Each eye projects to one magnocellular layer and 1 or 2 parvocellular layers; the 2 sets of geniculate layers project to separate subdivisions of layer $4 \mathrm{C}$ in the cortex; and the subsequent paths within area 17 are separate. Physiological studies in monkeys indicate that beyond areas 17 and 18 there exist multiple visual cortical areas, each with a complete topographic map of the visual field, and some evidence suggests that these prestriate areas subserve different aspects of vision: V4 has been reported to be particularly involved in color information (Zeki, 1980b) and MT with movement and stereopsis (Dubner and Zeki, 1971; Maunsell and Van Essen, 1983b). From their studies of temporal and parietal cortical lesions in monkeys, Pohl (1973) and Ungerleider et al. (1983) have proposed that higher cortical visual areas fall into 2 major subdivisions, one dealing with object recognition and the other with the location of objects in space ("where vs what"); as we will discuss later, their object recognition pathway probably represents the central continuation of the parvocellular pathway, and their localization pathway may correspond to the continuation of the magnocellular pathway. Clinical findings that very specific visual abilities can be selectively lost in half or even all of the visual field provide strong evidence that in humans, as well, different visual functions are carried out in separate brain regions. Localized cortical lesions can produce very selective types of blindness, such as the selective loss of the ability to recognize faces or the loss of color vision without loss of form vision. Lesions causing such specific visual deficits probably damage particular prestriate visual areas.

\section{Anatomical Pathways}

In the primate retinogeniculocortical pathway, a physical segregation of neurons differing in their physiological properties is first obvious in the latcral geniculate body, with its clear magnocellular and parvocellular subdivisions. These 2 sets of layers receive input from 2 major classes of retinal ganglion cells, A and B (Leventhal et al., 1981), which differ from each other anatomically and physiologically, even though they are not physically segregated (Boycott and Wässle, 1974). In the cortex 
the segregation begun in the geniculate is perpetuated, and the parvocellular system undergoes a further subdivision. Cells in the magnocellular layers project to layer $4 \mathrm{C} \alpha$ in area 17 (Hubel and Wiesel, 1972), which in turn projects to layer 4B (Lund, 1973; Lund and Boothe, 1975). Parvocellular cells project to $4 C \beta$ (Hubel and Wiesel, 1972), which in turn projects to the lower part of layer 3; from there the information is disseminated to the upper layers, 2 and 3 (Lund, 1973; Lund and Boothe, 1975).

Layers 2 and 3 are subdivided into a mosaic of blobs and interblobs, which are distinct from each other in their physiological properties, their local interconnections, and their projections to and from area 18 (Livingstone and Hubel, 1984a, b). Beyond layer $4 \mathrm{C}$, then, in the upper output layers of area 17, 3 anatomical/functional subdivisions can be distinguished: (1) layer 4B, (2) the interblobs of layers 2 and 3, and (3) the blobs of layers 2 and 3 .

Beyond area 17 these 3 pathways seem to remain segregated, and as far as we know do not become further subdivided. We will refer to the 3 pathways, for brevity (as opposed to elegance), as magno, parvo-interblob, and blob.

1. Magno. The stages in this pathway are the retinal $\mathrm{A}$ cells, magnocellular geniculate cells, and, in area 17, layers $4 \mathrm{C} \alpha$ and 4B; from 4B axons project to the midtemporal cortical area MT (Zeki, 1969; Lund et al., 1975; Spatz, 1975) and, as we have shown in the previous paper (Hubel and Livingstone, 1987), to the thick stripes of area 18. The thick stripes in area 18 also project to MT (DeYoe and Van Essen, 1985; Shipp and Zeki, 1985).

2. Parvo-interblob. This path begins with the $\mathrm{B}$ cells in the retina, proceeding to the geniculate parvocellular layers, and then to layer $4 \mathrm{C} \beta$ in area 17 . We think that $4 \mathrm{C} \beta$ provides the major input to the interblob regions, for somewhat indirect reasons: (1) Golgi studies (Lund, 1973; Lund and Boothe, 1975) indicate a strong and predominantly vertical projection from $4 \mathrm{C} \beta$ to deep 3; since interblob cortex occupies up to $90 \%$ of the surface area of 17 (Livingstone and Hubel, 1984a), it is hard to imagine that the projection from $4 \mathrm{C} \beta$ selectively avoids it; (2) the interblob cortex appears not to receive inputs either from the surrounding blobs or directly from the lateral geniculate body (Livingstone and Hubel, 1982, 1984b). Since, as described below, the physiological properties of interblob cells are in many respects different from the properties of cells in $4 \mathrm{C} \beta$ (orientation selectivity, high sensitivity to movement; relative rarity of overt color opponency), the conclusion that the major input to the interblob regions comes from $4 \mathrm{C} \beta$ must be based largely on anatomical evidence. From the interblob regions, one major forward projection is to the pale stripes of area 18 (Livingstone and Hubel, 1984a).

3. Blob. The cytochrome blob system, according to our present knowledge, can first be distinguished as a separate system only in the upper layers ( 2 and 3 ) of area 17 . The inputs to the blobs are still not known. They receive from the lateral geniculate body a weak projection (Livingstone and Hubel, 1982), which the results of Fitzpatrick et al. (1983) suggest originate in the interlaminar zones. The blobs may also receive input from layer $4 C \beta$ : there is no evidence that the projection from $4 C \beta$ into layers 2 and 3 selectively avoids the blobs, and, as discussed in the preceding paper (Hubel and Livingstone, 1987), the color properties of blob cells are not inconsistent with such a projection. As described below, the physiological properties of some blob cells suggest a contribution from magnocelluar type 4 cells
(Livingstone and Hubel, 1984a), a possibility that is supported by deoxyglucose studies using low luminance contrast (Tootell et al., 1985), and that is consistent with the sparing of the blobs after the apparently selective damage to the parvocellular system by acrylamide (Eskin and Merigan, 1986). The blobs send a strong forward projection to the thin stripes of area 18 (Livingstone and Hubel, 1984a).

Beyond area 18 , the projections of these 3 subdivisions are not fully worked out. According to Shipp and Zeki (1985), the thin stripes project to visual area 4 (V4), but DeYoe and Van Essen (1985) report that the input that V4 receives from 18 comes not only from the thin stripes but also from parts of the pale stripes. DeYoe and Van Essen (1985) and Shipp and Zeki (1985), in a rare instance of unanimity, find that MT receives projections from the thick stripes. The specificity of other prestriate projections, for example to $\mathrm{V} 3$, is not yet known.

Though the geniculocortical connections, the local inter- and intralaminar connections within areas 17 and 18 , and the corticocortical connections all suggest a remarkable segregation of these 3 systems, the separation may nevertheless not be absolute. The dendrites of cells in layer $4 \mathrm{~B}$ reach up into layers 2 and 3 (Lund and Boothe, 1975); some axons from $4 \mathrm{C} \alpha$ project to layer 2 (Fitzpatrick et al., 1985); the pulvinar, which receives inputs from 17 and 18 , projects strongly to upper-layer 17 and to the thick and thin stripes of 18 (see Curcio and Harting, 1978; Livingstone and Hubel, 1982; and Ungerleider et al., 1983, for review). Malpeli et al. (1981) recorded from cortical cells during reversible inactivation of different layers of the lateral geniculate and concluded that both simple and complex cells in area 17 can receive both magno and parvo input. Despite these and other possible exceptions, the degree of separation of the $3 \mathrm{di}-$ visions still seems to us quite impressive.

\section{Physiological Properties}

\section{Lateral geniculate body}

Differences in the physiological properties of cells in these 3 anatomical pathways indicate that they handle different kinds of visual information. Some of the most important differences probably originate in the retina, but they have been studied most extensively in the lateral geniculate body:

Color properties. Over $80 \%$ of parvocellular neurons show color-opponency, in effect subtracting the input of one cone type from that of another (or from the other two); they receive input from a single cone type over a relatively small region of the visual field (their receptive-field center) and antagonistic input from another cone type (or a sum of the other 2 cone types) from a larger area (the surround). Field centers of magnocellular neurons receive summating input from the red and the green cones, and probably also from the blue cones (see below), and are consequently termed "broadband"; they are therefore not usually considered to be color-coded. Many magnocellular neurons do show some color selectivity in their surrounds, in that they are tonically suppressed by diffuse red light but not by diffuse white light (De Valois et al., 1966, 1977; Wiesel and Hubel, 1966; Krüger, 1977; Schiller and Malpeli, 1978; Derrington et al., 1984).

Contrast sensitivity. Magnocellular neurons are more sensitive than parvocellular neurons to luminance contrast. As shown in Figure 1, they respond well to stimuli below $10 \%$ luminance contrast, whereas parvo neurons do not; the contrast gain (slope of the linear part of the curve of response vs contrast) is about 10 times higher; their responses saturate at lower contrasts 


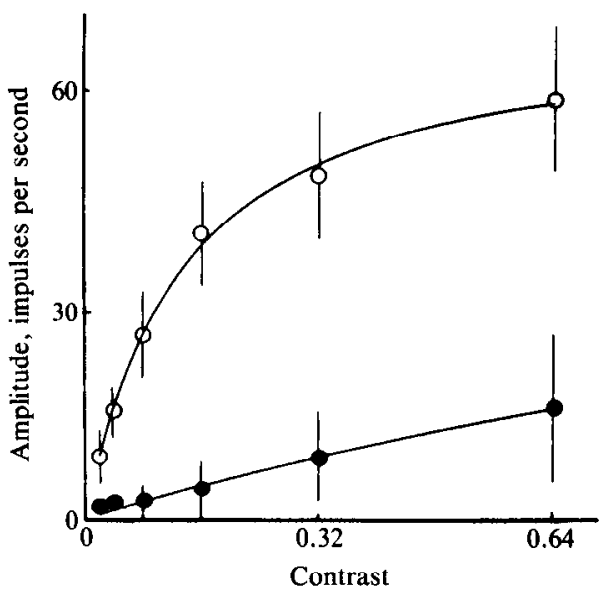

Figure 1. Average response versus contrast functions for 36 cells recorded from the lateral geniculate body of one rhesus monkey: 28 from the parvocellular layers (filled circles) and 8 from the magnocellular layers (open circles). The stimulus was a sinusoidal grating of optimal spatial frequency for each cell, drifting at $4 \mathrm{~Hz}$. Contrast is the difference divided by the average of the 2 components of the grating. From Kaplan and Shapley (1986).

(Shapley et al., 1981; Kaplan and Shapley, 1982, 1986; Derrington and Lennie, 1984).

Spatial resolution. Magnocellular cells have receptive-field centers 2-3 times larger, at any given eccentricity, than field centers of parvocellular cells (Derrington and Lennie, 1984).

Temporal properties. Magnocellular cells have a shorter latency than do parvocellular cells, and their responses decay more rapidly; consequently, they can follow higher frequencies of stimulation (Wiesel and Hubel, 1966; Dreher et al., 1976; Schiller and Malpeli, 1978; Hicks et al., 1983; Derrington and Lennie, 1984).

Tablc 1 summarizes the differences between the magno and parvo geniculate divisions. At least some of the physiological differences between the magno and parvo systems-differences in contrast sensitivity, spectral sensitivity, response time course, axon conduction velocity-are already present in the A and B retinal ganglion cells that project to these layers (Gouras, 1968, 1969; De Monasterio and Gouras, 1975; Schiller and Malpeli, 1978; Gouras and Zrenner, 1979; Leventhal et al., 1981; Kaplan and Shapley, 1986).

Merigan and Eskin (1986) have studied the temporal and spatial resolution of monkeys treated with a neurotoxin, acrylamide, that appears to damage selectively parvocellular geniculate neurons while sparing the magnocellular layers. Consistent with the physiological data summarized in Table 1, these mon- keys show a reduction in contrast sensitivity for high spatialand low temporal-frequency stimuli. Further studies on color vision and more complicated pattern-discrimination tests with these monkeys should be very interesting.

$X$ and $Y$ versus magno and parvo. Though the concept of separate and parallel paths for vision is an old one, the catchword "parallel processing" is comparatively recent, and has tended to be identified with the subdivision of cat retinal ganglion cells into 2 groups, $X$ and $Y$ (Enroth-Cugell and Robson, 1966). This identification is perhaps ironic, since in primates " $\mathrm{X}$ " and " $\mathrm{Y}$ " are probably not synonymous with "magno" and "parvo," for reasons that may be worth spelling out. The original distinction between $X$ and $Y$ cells was based on linearity of spatial summation within the receptive-field center. $X$ and $Y$ cells, when distinguished by that criterion, usually differ in additional ways: $X$ cells, compared to $Y$ cells, tend to have smaller cell bodies and axons; their field centers tend to be smaller; their responses to light tend to be more sustained. In the monkey, the magno- and parvocellular subdivisions of the geniculate share some of these subsidiary distinctions: cells in the magno layers, compared to parvocellular cells, have bigger cell bodies and axons, their field center sizes are larger, and they give briefer responses when their field centers are illuminated. As reviewed by Shapley and Perry (1986), the 2 ways of subdividing cells, $\mathrm{X}$ or $\mathrm{Y}$ versus magno or parvo, nevertheless differ in at least 3 important ways: (1) Far from being segregated in the lateral geniculate body, $X$ and $Y$ cells are intermixed, both in the $A$ and $\mathrm{A} 1$ layers in the cat, and in the magno layers in the monkey (Blakemore and Vital-Durand, 1981; Shapley et al., 1981). The cortical projections of $\mathrm{X}$ and $\mathrm{Y}$ cells in the cat are to some extent separated, but are not known to be in the monkey. (2) In the monkey the most striking difference between magno and parvo cells is in color coding, but there is no clear distinction between the color properties of $\mathrm{X}$ and $\mathrm{Y}$ cells in cats or in monkeys. (3) In the monkey, magno and parvo cells show marked differences in contrast sensitivity, but $\mathrm{X}$ and $\mathrm{Y}$ cells do not either in monkeys or cats (Kaplan and Shapley, 1982).

Magno cells in the monkey would seem to be homologous to the cells in the dorsal (A/A1) pair of layers in the cat, in that both show high contrast sensitivity, contain $X$ and $Y$ cells, and develop early (for references, see Kaplan and Shapley, 1982). The occurrence of occasional color-opponent cells in the C complex in the cat is the only hint that the $C$ layers could be homologous to the parvocellular layers in the monkey (Daw and Pearlman, 1970).

In summary, while we cannot rule out the possibility that in primates $\mathrm{X}$ and $\mathrm{Y}$ cells represent separate pathways beyond the lateral geniculate, we know of no strong physiological or anatomical evidence to support it, whereas there is conclusive evi-

Table 1. Physiological properties of the magno and parvo geniculate divisions

\begin{tabular}{lll} 
& Geniculate subdivision & \\
\cline { 2 - 3 } Property & Parvocellular & Magnocellular \\
\hline Color & Yes (color-opponent) & No (broadband) \\
Contrast sensitivity & Low (threshold $>10 \%)$ & High (threshold $<2 \%)$ \\
Spatial resolution & High & $\begin{array}{c}\text { Lower (by 2-3-fold at a given } \\
\text { eccentricity) }\end{array}$ \\
Temporal resolution & Slow (sustained responses, low & Fast (transient responses, high \\
& conduction velocity) & conduction velocity) \\
\hline
\end{tabular}


dence to support a segregation of magno and parvo pathways. It seems unlikely that $X$ and $Y$ channels - if they are channelsare the same as magno and parvo channels, and we feel that to term the magno system " $Y$ " and the parvo " $X$ " goes beyond what is known, and is probably wrong.

\section{Cortex}

The properties of cortical cells in each of the pathways must of course derive from the properties of their geniculate ancestors, but the relationship is not always clear, and the appropriate questions have not all been asked. For each stage in each of the 3 pathways we will attempt to summarize what is known about their physiological properties, in particular how these properties might be related to the 4 criteria, listed in Table 1 , that differentiate between magno and parvo geniculate cells.

The magno system (magno $\rightarrow 4 C \alpha \rightarrow 4 B \rightarrow$ thick stripe $\rightarrow$ MT)

This system displays orientation selectivity at its earliest cortical stage, in layer $4 \mathrm{C} \alpha$ in 17 , as well as at subsequent stages: in layer 4B of 17, the thick stripes of 18 , and MT (Dubner and Zeki, 1971; Dow, 1974; Poggio and Fischer, 1977; Zeki, 1980a, 1983; Maunsell and Van Essen, 1983a; Blasdel and Fitzpatrick, 1984; Hawken and Parker, 1984; Livingstone and Hubel, 1984a; Allman et al., 1985a; Poggio et al., 1985). In layer 4B many cells also show direction selectivity (Dow, 1974; Livingstone and Hubel, 1984a); many of them are binocular (Hawken and Parker, 1984), and many are selective for stereoscopic depth (G. F. Poggio, personal communication). Cells in the thick stripes of 18 seem particularly involved in stereoscopic depth: about two-thirds are very precisely tuned for binocular disparity, and cells with like disparities are clustered together (previous paper, Hubel and Livingstone, 1987). Most cells in thick stripes respond only to moving stimuli, but show no marked tendency to be direction-selective. Area MT is often spoken of as a "movement area," becausc of a high prevalencc of dircction sclcctivity, with cells of similar directionality clustered together; but other observations suggest that MT has a broader function than just movement detection: it is concerned with textures and figureground relationships (Allman et al., 1985b), and many of its cells are highly selective for binocular disparity (Zeki, 1973, 1980a; Maunsell and Van Essen, 1983b).

The parvo-interblob system (parvo $\rightarrow 4 C \alpha \rightarrow$ interblobs $\rightarrow$ pale stripes)

Cells in $4 \mathrm{C} \beta$ are similar to parvocellular geniculate cells in having very small, circularly symmetrical receptive fields with center-surround antagonism, but one difference seems to be that color-opponency may be less prominent or explicit (Bullier and Henry, 1980; Blasdel and Fitzpatrick, 1984). At the next stage of processing, in the interblob regions of layers 2 and 3 of area 17 , the cells are quite different from cells at previous stages, in being precisely tuned for stimulus orientation, mostly complex, and mostly unselective for stimulus color. They strongly prefer moving stimuli and do not respond well to flashed stationary stimuli. In the pale stripes of 18 , cells resemble interblob cells, except that a high proportion are, in addition, end-stopped (they respond to short but not long bars).

The blob system $($ blob $\rightarrow$ thin stripe $\rightarrow$ V4)

Our knowledge of the physiology of the blob system begins with the blob cells themselves, since we are uncertain of their input.
The evidence mentioned above suggests that they receive both magno- and parvocellular input. Unlike other upper-layer cells, blob cells are not selective for stimulus orientation, and most have center-surround receptive fields (a few are center-only without an antagonistic surround). In the macaque, roughly half of the blob cells are color-coded, with color-opponent centers and antagonistic color-opponent surrounds. The remainder are broadband center-surround. We think that the blob system analyzes color, with the broadband cells conveying information on brightness (Livingstone and Hubel, 1984a). In area 18, the cells in the thin dark stripes are similar to those in the blobs in lacking orientation selectivity and showing a high incidence of coloropponency, but many are complex in the sense that they respond to a stimulus over a larger region of the visual field without an increase in optimum stimulus size (Hubel and Livingstone, 1985).

We now ask in more detail how the physiological differences between the magno and parvo geniculate systems, discussed above, relate to the properties of cells at more central stages in each of these systems.

Color properties. Cells in layers $4 \mathrm{C} \alpha$ and $4 \mathrm{~B}$, like their predecessors in the magnocellular geniculate layers, have broadband receptive fields (Blasdel and Fitzpatrick, 1984; Livingstone and Hubel, 1984a).

In the cortical continuation of the parvocellular system, it is not clear what happens to the preponderance of color coding seen in the geniculate. In the interblobs, overt color-opponency is surprisingly rare, occurring in only about $10 \%$ of the orientation-specific cells (Hubel and Wiesel, 1968). If interblob cells receive their input mainly from layer $4 \mathrm{C} \beta$, and hence from the parvocellular geniculate layers, they must have lost the explicit (wavelength- and sign-specific) color coding present in those layers. Many interblob cells do, nevertheless, seem to carry some kind of color information. Gouras and Krüger (1979) found that of the orientation-selective cells in layers 2 and 3, most (78\%) were contrast-sign invariant, in that a black-white line or edge that evoked a response continucd to cvoke a response when the contrast was reversed. About half of such cells, even though not overtly color-coded (no demonstrable opponency; good responses to white light), responded to a color-contrast border at all relative settings of intensities of the 2 colors. Gouras and Krüger suggest that color-coded inputs are somehow pooled in such a way that information about the sign of the color-opponency and also the sign of black-white contrast is lost, but responsiveness to color contrast and to luminance contrast is retained.

For the blobs, a comparison of geniculate and cortical properties is more speculative, since we are uncertain of their inputs. Color-opponent blob cells must derive their color properties from color-selective geniculate inputs, which could be the parvocellular neurons, since, as discussed in the accompanying physiology paper (Hubel and Livingstone, 1987), a double-opponent organization is not incompatible with a parvocellular type 1 input, given the larger size of double-opponent field centers. We have recorded from a few blob cells that have properties very similar to those of magnocellular type 4 cells (Wiesel and Hubel, 1966; Livingstone and Hubel, 1984a). Thus the color properties of blob cells would be compatible with input from both magno and parvo systems.

Contrast sensitivity. The differences in contrast sensitivity of the 2 geniculate divisions seem to be perpetuated straightforwardly in the cortex. Cells in $4 \mathrm{C} \alpha$ have higher contrast sensitivity than do cells in 4 C $\beta$ (Blasdel and Fitzpatrick, 1984; Haw- 


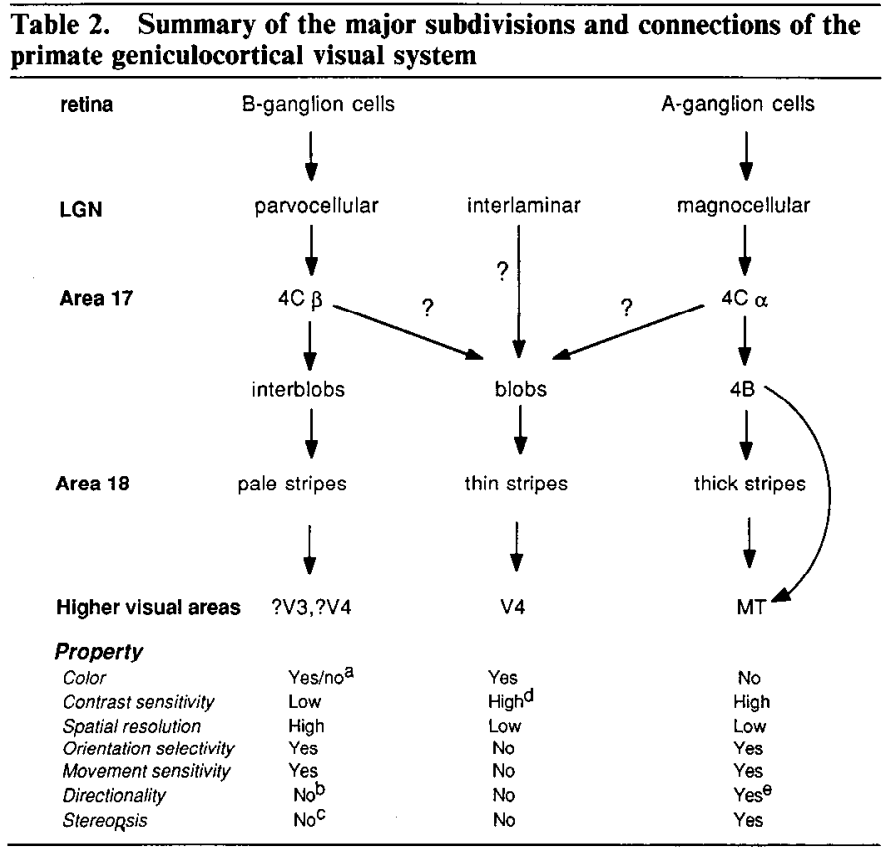

a Cells beyond $4 \mathrm{C} \beta$ do respond to color-contrast borders but are not overtly colorcoded.

${ }^{b}$ At least it is not prominent.

"In anesthetized animals, we have seen only a few stereotuned cells in upper-layer area 17 (Livingstone and Hubel, 1984b; see also Hubel and Wiesel, 1970). In attentive animals, cells coded for stereoscopic depth have been reported both above and below layer $4 \mathrm{C}$ of area 17 , but are especially concentrated in layer $4 \mathrm{~B}$ (Poggio and Fischer, 1977; Poggio et al., 1985; G. F. Poggio, personal communication). We do not understand these differences in results, but one possibility is that the stereo mechanisms are built up in 18 , and the stereotuning in 17 is the result of a back projection that is suppressed by anesthesia.

${ }^{\top}$ By deoxyglucose (Tootell et al., 1985)

- Rare in thick stripes in area 18 but very common in layer $4 \mathrm{~B}$ of area 17 and in MT.

ken and Parker, 1984). Studies using deoxyglucose (Tootell et al., 1985) indicate that cells of the magno path retain their high contrast sensitivity at more central levels: Stimulation with stripes of $8 \%$ contrast did not produce stimulus-dependent uptake in the parvocellular geniculate layers, or in layer $4 \mathrm{C} \beta$ or the interblobs in 17 , but did result in stimulus-dependent labeling of the magnocellular layers of the geniculate, layers $4 \mathrm{C} \alpha$ and $4 \mathrm{~B}$ in area 17, and the higher visual area MT; the blobs in area 17 were also labeled, again suggesting that they too may receive some magnocellular input. Alternate dark stripes in area 18 were labeled, but in the macaque it was not possible to distinguish whether they were thick or thin.

Spatial resolution. The differences in spatial resolution of the 2 geniculate subdivisions are also apparent at the next stage, layer 4 of area 17. Receptive-field centers of cells in layers $4 \mathrm{C} \alpha$ and $4 \mathrm{~B}$ have diameters 2 or more times as large as field centers in $4 \mathrm{~A}$ or $4 \mathrm{C} \beta$ (Blasdel and Fitzpatrick, 1984). Receptive fields of interblob cells seem to be consistent with a fine-grained parvo input, since the optimum stimulus size is also tiny. The resolution of blob cells is coarser, with receptive-field center diameters at least twice as large as the optimum linewidths for interblob cells at the same eccentricity (Livingstone and Hubel, 1984a).

Temporal properties. As with color, differences in temporal properties of the 2 geniculate divisions do not seem to be perpetuated in any simple way in the cortex. The faster, transient responses of the magno system would make it well suited for movement perception, and cells in magno-recipient layer $4 \mathrm{~B}$ indeed prefer moving stimuli, and many are direction-selective. But cells in the parvo-recipient interblobs also prefer moving stimuli over stationary ones, though they are not as often direction-selective. The end-stopped cells of the pale stripes in 18 are similarly very sensitive to movement. Blob cells, on the other hand, respond well to stationary stimuli, often giving sustained responses to appropriately colored spots, and show no direction selectivity; they seem not to be concerned with movement.

The major anatomical and physiological distinctions of thesc pathways are summarized in Table 2 . Taken together, the physiology and anatomy suggest that we can assign the following properties and functions to the 3 anatomical subdivisions:

1. The magnocellular $\rightarrow 4 B \rightarrow$ thick $\rightarrow$ stripe $\rightarrow$ MT pathway exhibits systematic selectivity for movement and binocular disparity, suggesting that this system is especially concerned with depth and motion perception. At the cortical stages, most, if not all, the cells are orientation-selective, suggesting that this system could also carry information about edge orientation, but the physiology does not indicate whether this information is used for the analysis of shape or for resolving ambiguities in stereo and motion matching, or both. This system has high temporal resolution, high contrast sensitivity, relatively low spatial resolution, and is probably color-blind.

2. The parvocellular $\rightarrow$ interblob $\rightarrow$ pale $\rightarrow$ stripe system is characterized by high spatial resolution, orientation selectivity, and end-stopping. Since we do not (yet) know of any more specific or illuminating physiological properties of this system, we can only guess that it is involved in high-resolution form perception. Though interblob cells may respond to color-contrast borders, they are probably not involved in the perception of color as such, since most of them are not wavelength- or contrast-sign-selective.

3. The blob $\rightarrow$ thin stripe $\rightarrow V 4$ system is concerned with color, but not with movement or stereopsis. Its resolution is low, and its lack of orientation tuning as far centrally as area 18 suggests that it is not important in form perception.

The physiological properties of these functional divisions should have some obvious psychophysical consequences, and the goal of the present study was to test such predictions by reviewing the psychophysical literature, repeating some experiments, and doing some of our own. We asked if the psychophysics of color and form vision, as compared with that of depth and movement, is consistent with the physiological differences between the parvocellular and blob systems, on the one hand, and the magnocellular system on the other. For example, are movement and depth perception, as compared to high-resolution form perception, less sensitive to color, more sensitive to luminance contrast, and do they have lower spatial and higher temporal resolution?

\section{Materials and Methods}

For most of the psychophysical experiments to be described, we used a color graphics system consisting of a color monitor and graphics equipment (Raster Technologies $1 / 25 \mathrm{M}$ ) driven by a Hewlett-Packard 9000 computer. Some of the black-and-white displays were generated with a Hewlett-Packard 9826 desktop computer.

The color monitor phosphors (P22) had the characteristics shown in Table 3. Compared to monochromatic red at $630 \mathrm{~nm}$, the red phosphor by itself appeared somewhat orange, and looked more like monochromatic $610 \mathrm{~nm}$, because of several shorter-wavelength components peaking at 590 and $540 \mathrm{~nm}$. We did not use an achromatizing lens for our 


\begin{tabular}{|c|c|c|c|}
\hline & Red & Green & Blue \\
\hline \multicolumn{4}{|c|}{ Wavelength at peak energy (nm) } \\
\hline & 630 & 525 & 445 \\
\hline \multicolumn{4}{|c|}{ CIE chromaticity coordinates } \\
\hline$x$ & 0.61 & 0.31 & 0.15 \\
\hline$y$ & 0.35 & 0.59 & 0.07 \\
\hline
\end{tabular}

experiments because we found that many phenomena either were greatly diminished or disappeared entircly at some ratio of red to green. Wc assume this means that, at least for those visual tasks, any borders caused by chromatic aberration were insignificant.

Light intensities were determined with a Pritchard spot photometer (Spectra, Model 1980A CD). The light energy from the monitor screen was higher in the center of the screen than at the edges by about $20 \%$, and these variations were parallel for the 3 different phosphors. To simplify measurements of acuity and equiluminance determinations as a function of eccentricity, we therefore kept test spots at one screen position and varied the gaze-fixation point. When intensities were critical for different positions on the screen, as in studies of apparent movement at equiluminance, separate adjustments and measurements were made for each of the spots. We have expressed the luminance contrast between a stimulus and the background as a percentage, equivalent to the Wcber fraction; that is, $\left(L_{\text {test }} L_{\text {bkg }}\right): L_{\text {bkg }} \times 100$. For gratings we expressed contrast as the difference in luminances divided by the average: $\left(L_{\max }-L_{\min }\right) \div\left(L_{\max }+L_{\min }\right) / 2$. The refresh rate for the monitor was $16 \mathrm{msec}$, so the maximum alternation rate was $30 \mathrm{~Hz}$ and, unless otherwise noted, interstimulus intervals were $16 \mathrm{msec}$.

We made most observations in a room illuminated by dim overhead tungsten lamps, just bright enough so that colors of objects could easily be seen. A white paper placed at the level of the color monitor had a luminance of $0.03 \mathrm{~cd} / \mathrm{m}^{2}$. For some observations, in order to rule out any significant rod contribution, we used the overhead incandescent lights, which brought the luminance of the paper to $2.5 \mathrm{~cd} / \mathrm{m}^{2}$.

Most of the observations were made on ourselves, but students and visitors were often subjects. Graeme Mitchison acted as our arch-skeptic in cases where we had any doubt as to the results; he could usually be relied on to see stereoscopic depth when everyone else failed. We have shown many of these experiments in public demonstrations, with the results confirmed by hundreds of observers.

\section{Results (New and Old)}

Since physiological studics had suggested that the magnocellular pathway is color-blind, we wanted to test psychophysically as many visual functions as possible under conditions in which there were color but no luminance differences across borders or between figures and background. It should be emphasized that we use the term "equiluminance" to refer to that balance of 2 wavelengths, always red and green in this paper, at which some psychophysical phenomenon reaches a maximum or minimum. We will see that the red-to-green ratio at which this happens is highly variable from person to person, from phenomenon to phenomenon, and even from one retinal eccentricity to another.

One of the most striking differences between magno and parvo geniculate neurons is in their responses to color. As a way of distinguishing between visual abilities attributable to one or the other of these 2 systems, we have therefore used the technique of making visual stimuli equiluminant. An ability that breaks down at equiluminance probably depends on a broadband system for the following reasons: As a green/red edge is moved across the receptive-field center of an on-center broadband cellone that receives excitatory input from both red and green conesgreen-cone excitation will decrease but red-cone excitation will increase, and the resultant firing rate will depend on the relative

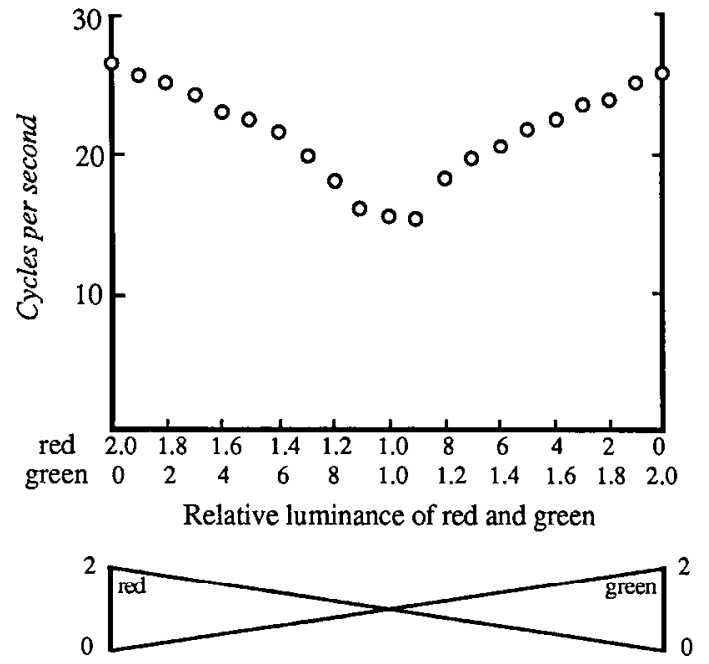

Critical speeds for disappearance of flicker for various relative amounts of alternated red and green light. From Ives, 1923.

Figure 2. Maximum rates for seeing flicker with alternating red and green spots, as a function of relative brightness of the red and green.

stimulation of the red and green cones by the 2 colors. At some setting of relative brightness of red and green, the cell should show no change in firing rate when the border crosses its receptive field in either direction of movement. For that cell we can say the red and green are then equiluminant. On the other hand, a color-opponent cell, say red-on/green-off, should respond even better to a green-red border than to a black-red border, summing excitation from red-cone input and the removal of inhibition from green. Balancing the brightness of 2 colors should not result in a response minimum in color-opponent cells.

By this reasoning, magnocellular cells should show a response minimum to colored borders at some relative brightness, whereas color-opponent parvo cells should not. Two physiological studies seem to bear this out. Krüger (1979) found that $75 \%$ of the magno geniculate units he studied were unresponsive to moving color-contrast stimuli at some setting of relative brightness, and this critical setting was about the same for all the cells he studied. Hicks et al. (1983) found that magno cells responded "poorly or not at all" to chromatic gratings, whereas coloropponent parvo cells responded better to color-contrast gratings than to luminance-contrast gratings (see also De Valois et al., 1977). The opposite conclusion was reached in a third study, by Schiller and Colby (1983), in which substitution of a stationary spot of one color by a spot of another color spot produced responses at all relative intensities. The different results may be attributable to the use of stationary color substitution rather than moving color borders (see Krüger, 1979).

We have made much use of one way of measuring equiluminance, flicker photometry, because of its consistency in any one subject at a given eccentricity, and because it is easy to measure.

\section{Color flicker photometry}

When a test stimulus alternates between 2 values that differ in brightness or in color, the alternation can only be perceived below a critical frequency. For brightness differences, this rate can be as high as 70 cycles/sec. For color alternations without 


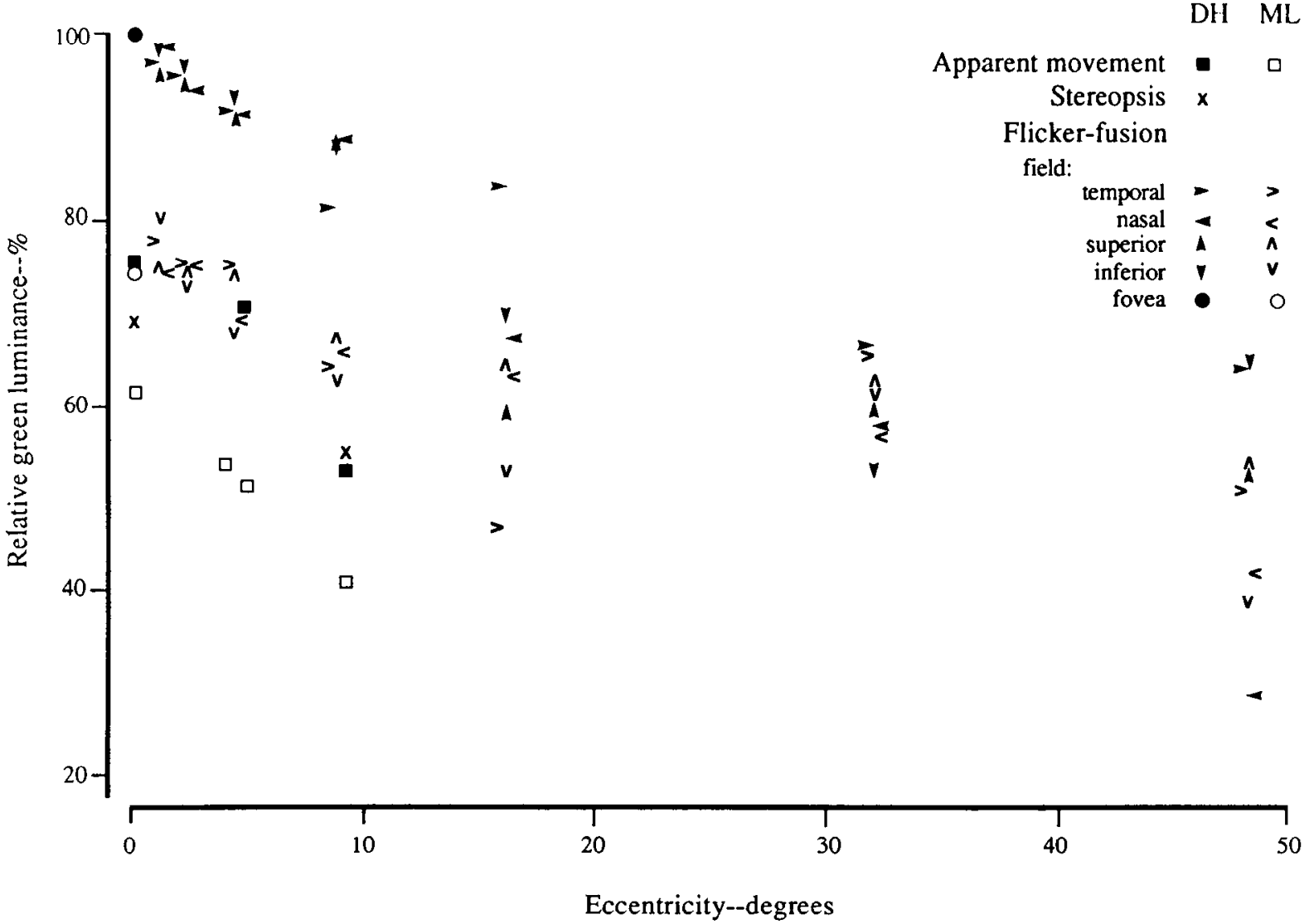

Figure 3. Relative amounts of green required for a fixed intensity of red to achieve loss of flicker for an alternation of red and green at $15 \mathrm{~Hz}$ (arrowheads), loss of apparent movement (squares), and loss of stereopsis $(\times s)$ for 2 different observers (D.H. and M.L.), plotted against eccentricity (distance from center of gaze, in degrees). For stereopsis and apparent movement, the points plotted all were in the inferior visual field along the vertical meridian; for flicker fusion we made measurements out from the center of gaze in 4 directions (filled and open arrowheads). The foveal value for the amount of green for observer D. H. for flicker fusion was taken as 100 , and other values expressed relative to this as percentages. Since the brightness of our color monitor was not constant across the screen, we varied the fixation point and kept the stimulus position constant.

any brightness differences, the highest frequency at which alternations can be detected (the color-contrast flicker-fusion frequency) is about $10 / \mathrm{sec}$ (Ives, 1923). For example, if a test stimulus alternates between red and green at frequencies between 10 and $30 \mathrm{~Hz}$, the color alternation cannot be seen, so the colors fuse, producing yellow or brown; but if the red and green are not equally bright, a sensation of flicker persists (the luminance flicker), and one perceives an alternation between dark and light yellow. If one now varies the relative brightnesses of the red and green, the luminance flicker disappears over a narrow red-to-green range. For a pair of colors, the ratio of intensities at which flicker disappears provides one definition of equiluminance for that pair of colors (Fig. 2).

The fact that color-contrast flicker fusion occurs at much lower frequencies than luminance-contrast flicker fusion suggests that the color system is slower than the luminance system, and could reflect the higher temporal resolution of the magnocellular system, compared with the parvocellular or blob system.

To measure flicker-photometry equiluminance, we generated on the color monitor screen a rectangle that alternated color from red to green at a frequency of 15 cycles/sec. The size of the rectangle was $1 / 2^{\circ}$ in subtense for foveal measurements, and was made larger for peripheral measurements. We set the output of the less bright phosphor to an intermediate value and changed the intensity of the other phosphor continuously until no visible flicker could be scen. For most observers, at small eccentricities the setting was very critical, but between observers it varied over a wide range. For one of us the loss of flicker required appreciably more green, which we attribute to the increasing yellowness of the lens known to attend advancing years.

We noticed that if we fixed our gaze on a small $\left(1 / 2^{\circ}\right)$ alternating spot and adjusted the red-green ratio for zero or minimal flicker, and then looked away from the spot, the flicker reappeared. At first we dismissed this by assuming that our peripheral vision was more flicker-sensitive, but to our surprise we found that with our gaze deviated by some fixed amount, we could readjust the setting so that we saw no flicker, and when we then looked back directly at the spot, the flicker reappeared. We therefore decided to measure our equiluminance ratios as a function of eccentricity. Results for the two of us are given in Figure 3. At any given eccentricity, D.H. required more green than M.L. to balance a given red setting, but for both of us the amount of green required to balance a given red decreased with eccentricity. The major variation occurred in the first few degrees. Earlier studies have reported similar variations in color sensitivity with eccentricity for both flicker photometry and color matching, and have attributed these differences to absorption by the macular 
pigment (Hering, 1893; Naylor and Stanworth, 1954; Stabell and Stabell, 1980; Viénot, 1983), but it is possible that some of the differences may reflect changes with eccentricity in the relative numbers of the different cone types (Marc and Sperling, 1977). In either case, the results suggested that experiments testing the effect of equiluminance on particular visual tasks should take into account variations in equiluminance with eccentricity and from subject to subject. In addition, as we show below, equiluminance settings for minimal flicker fusion did not generally agree with those for minimizing other phenomena. The fact that many visual abilities are diminished, or even fail entirely, at some equiluminance point could be taken to imply that most or all of the cells responsible for those abilities are minimally responsive at the same luminance ratio. Perhaps subjects who see the least effects of equiluminance have the most variation in individual cells' equiluminance points within their population of broadband cells.

\section{Movement \\ Contribution of color-contrast and luminance-contrast information to movement}

We have listed reasons for suspecting that the magno system is highly sensitive to movement. If this system were solely responsible for our perception of movement, we would predict, from physiological studies in monkeys, that humans should be able to detect movement of very low luminance-contrast stimuli but not of equiluminant stimuli. We will begin by describing several experiments that suggest that color information does indeed contribute little, if at all, to some aspects of movement perception.

Cavanagh et al. (1984) compared equiluminant moving colorcontrast sine-wave gratings to moving luminance-contrast gratings for their ability to produce a subjective experience of movement. They found that "the perceived velocity of equiluminous gratings is substantially slowed at low spatial frequencies. The gratings often appear to stop even though their bars are clearly resolved. In these instances, the motion is appreciated only because it is occasionally noticed that the bars are at some new position." We repeated their experiment by generating on an oscilloscope screen horizontal red/green sine-wave gratings of $0.5-3$ cycles/deg, with the red and green components $180^{\circ}$ out of phase, moving downwards at a constant rate of 1 cycle/sec. When we varied the relative brightness of the red and grecn components, we also found a broad range over which the perceived movement slowed markedly, or even appeared to stop altogether. This range included isoluminance, as defined by flicker photometry, and extended about $5 \%$ above and below it. If we placed a piece of red cellophane over one side of the screen, producing a red/black luminance-contrast grating on that side, the black and red stripes appeared to move much faster than equiluminant green and red stripes. Similarly, moving patterns of green dots on a red background or red and green square waves also appeared to move much more slowly at equiluminance.

Even if the movement system depended solely on luminance contrast, one might not predict that the result of minimizing luminance contrast by using equiluminant colors would be a slowing of the perceived movement (as opposed, say, to making the movement less vivid). Of course, in some sense amount of movement is speed. Indeed, Campbell and Maffei (1981) and Thompson (1982) found that the perceived rate of movement of gratings depends on the luminance contrast: At slow rates of movement, decreasing the luminance contrast slows the appar- ent rate of movement; at high rates of movement, decreasing the contrast increases the perceived velocity. This correlates with the behavior of most movement-sensitive cells, whose responses decline for movements faster or slower than their optimum rate, but also decline at low contrasts.

We were shown a similar demonstration by E. Land (Fig. 4), consisting of 2 disks side by side, geared to rotate in opposite directions at the same speed, one with alternating vanes of 2 shades of gray that differed in brightness by only $10 \%$, and the other with vanes of $100 \%$ contrast. The disk with the lower luminance contrast appeared to be rotating 2-4 times slower than its companion, and actually appeared to be stationary when we looked a few degrees away. We could nevertheless easily deduce that the 2 disks were rotating at exactly the same speed by watching the 2 sets of vanes go by one another and seeing that they were always in phase.

Next we asked whether apparent, or phi, movement is similarly dependent on luminance contrast, and not seen with pure color contrast stimuli. The phenomenon of apparent movement was described by Wertheimer (1912), who found that flashing 2 spatially separate stationary stimuli sequentially can result in the perception of motion in the absence of any real motion. This effect is now commonly used in neon signs, airline landing strips, and cinematography. There is some question whether perceiving apparent movement and perceiving real movement depend on the same neural mechanisms (for discussion of longvs short-rangc movement, see Braddick, 1974; Anstis, 1980), but a study by Gregory and Harris (1984) showing that real and apparent movements of opposite direction can cancel suggests that they have some elements in common. In studies of apparent motion of equiluminous kinematograms and equiluminous bar stimuli, Ramachandran and Gregory (1978) reported that apparent motion disappeared at equiluminance for random-dot kinematograms but not for simple line stimuli, whereas Cavanagh et al. (1985) reported that apparent movement did disappear for simple line stimuli, but only for short interstimulus intervals.

As far as we know, such experiments have not been done under conditions in which possible variations in equiluminance with eccentricity have been taken into account. We therefore set up an apparent-movement display in which we could vary independently the brightness of 2 spatially separate, alternately flashed green spots on a red background. We asked the subject to direct his gaze at a fixation point and to adjust first one and then the other spot until the sensation of movement was eliminated or minimized. Spots of $12^{\circ}$ alternated position between $0^{\circ}$ and $3^{\circ}$ eccentricity or between $1^{\circ}$ and $4^{\circ}$ eccentricity, and $1^{\circ}$ spots alternated between $4^{\circ}$ and $8^{\circ}$ eccentricity. We tested alternation rates of 1,2 , and $3 / \mathrm{sec}$, with interstimulus intervals of $16 \mathrm{msec}$. For each spot position, we measured directly the luminance for red and for green at which apparent movement was lost, and compared that to the red/green ratio for flicker fusion, as described above. To make the conditions as similar as possible, flicker fusion was tested on the same red background. We found that apparent movement did indeed disappear, but only when both spots were individually set to critical $\mathrm{red} / \mathrm{green}$ ratios. All traces of movement then disappeared and the spots seemed to dance alternately on and off. When one spot was foveal, it required a higher green/red value, consistent with the variation of equiluminance with eccentricity described above. The results are shown by the squares in Figure 3. For each of us, and for each eccentricity, equiluminance defined in this way 

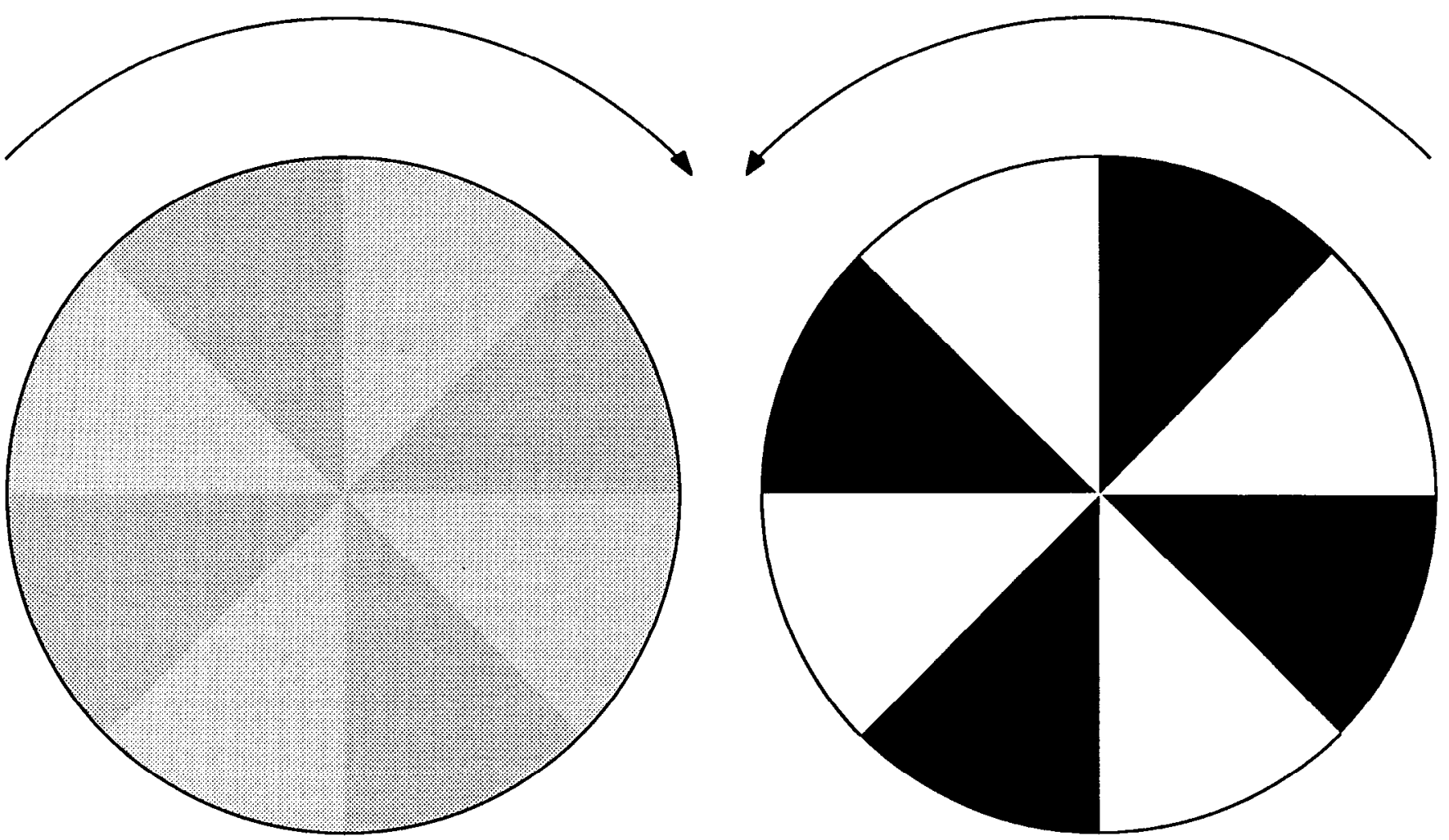

Figure 4. Stimulus used to demonstrate deterioration of movement perception at low luminance contrasts. Two disks, one with vanes differing in brightness by $100 \%$, the other with vanes differing by $10 \%$, counterrotated slowly $(5 \mathrm{rpm})$ at equal rates, but the left-hand disk appeared to rotate much more slowly. Naive observers reported that the higher-contrast disk was rotating 2-4 times faster.

was obtained at settings for green about $20 \%$ lower than those obtained with flicker photometry. The dancing quality of the spots at equiluminance may be related to a phenomenon we have noticed in many of these experiments: when a green spot on a red background, or the reverse, is turned off, it is followed by a bright complementary afterimage, which is most striking when the colors are equiluminant.

These results agree with previous work (Campbell and Maffei, 1981; Thompson, 1982; Anstis and Cavanagh, 1983) in showing that for movement perception luminosity differences are much more important than color differences. Indeed, for apparent movement, our results give no indication of any contribution from color-contrast information, though they do not rule it out. But they do suggest that to be sure of the reverse conclusionthat color does contribute to movement-one must take into account variations in equiluminance with eccentricity.

We tested the contribution of color to movement processing in another way, by asking whether color coding could resolve ambiguities in apparent movement. Kolers and Pomerantz (1971) found that the probability (as a function of interstimulus interval) of seeing apparent motion between 2 alternately presented stimuli is not strongly influenced by either the form or the color of the stimuli. Gengerelli (1948) developed an apparent-motion display in which pairs of spots on diagonally opposite corners of a square are alternately turned on and off (Fig. 5). In such a display, the direction of motion is ambiguous: one can see either vertical or horizontal apparent motion, and the perception is metastable, like a Necker cube (Necker, 1832). There is hysteresis in the perception; one tends to see one axis of motion for a few seconds; then the percept suddenly switches. We used such a square to ask whether the color or form of the spots has any influence on the direction of the apparent motion. A square arrangement of dots produces apparent motion with some slight preference for vertical over horizontal movement, whereas with a $45^{\circ}$ diamond arrangement neither axis is preferred (Gengerelli, 1948; Ramachandran and Anstis, 1983). With either arrangement, making 2 of the dots red and 2 green had little or no influence on the direction of the perceived motion (Fig. 5, second row); with 2 upper dots red and 2 lower ones green, vertical movement was still preferred, and with the diamond arrangement the perception of a red dot moving and simultaneously turning into a green dot and back to red was experienced as often as the perception of a red dot and a green dot traveling back and forth independently (Gengerelli, 1948). This was so regardless of the relative intensities of the red and green spots, which we could vary continuously through equiluminance, as estimated by flicker photometry. (When the spots are equiluminous with the background the situation is quite different, sincc then, as already discussed, apparent movement is not seen at all.) We could have quantified this apparent lack of influence of color on apparent movement, as, for example, Ramachandran et al. (1986) and Chaudhuri and Glaser (1986) have done in examining the influence of interspot distance on axis preference, but we resisted the temptation because the problem is complicated by hysteresis and by variables such as distance, brightness, and interstimulus interval, which went beyond our immediate interests. Our conclusion is that the influence of color is weak enough that careful quantification would probably be necessary to reveal it, and supports the earlier conclusions of Gengerelli (1948) and Kolers and Pomerantz (1971).

We further asked whether, in a nonchromatic display, the amount or sign of the luminance contrast would influence the 

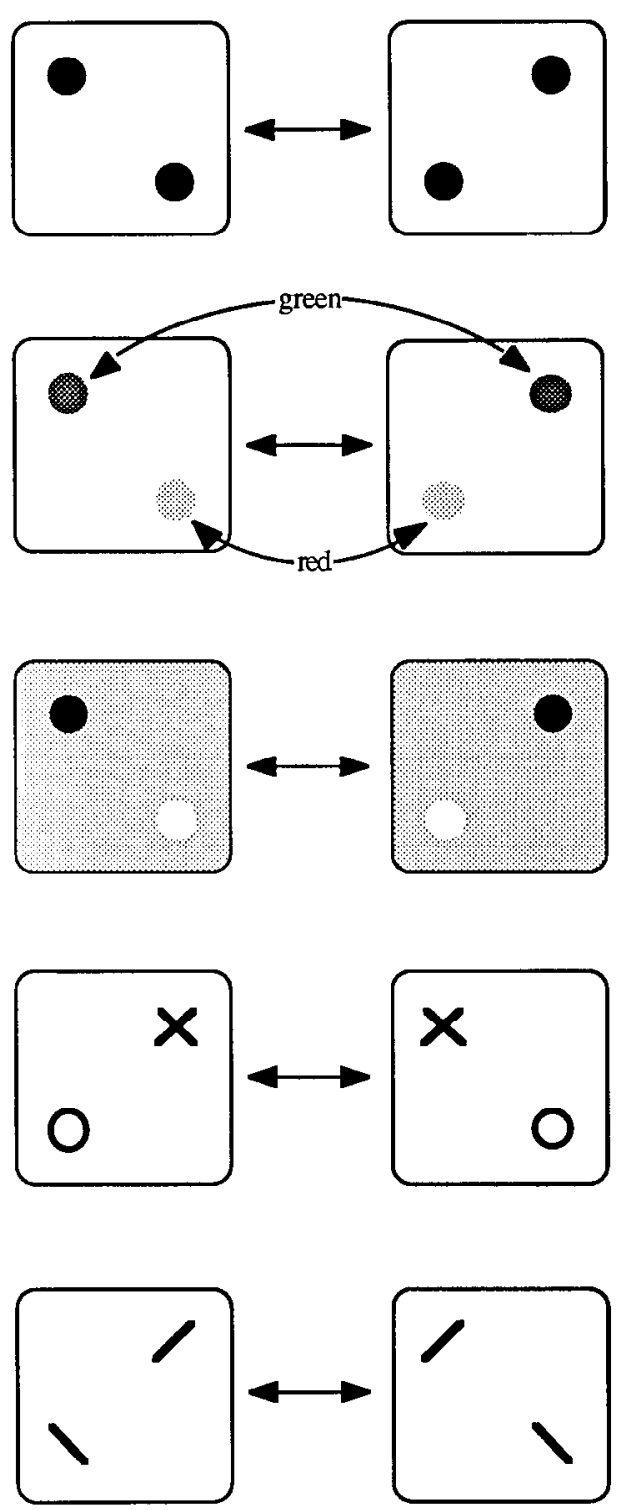
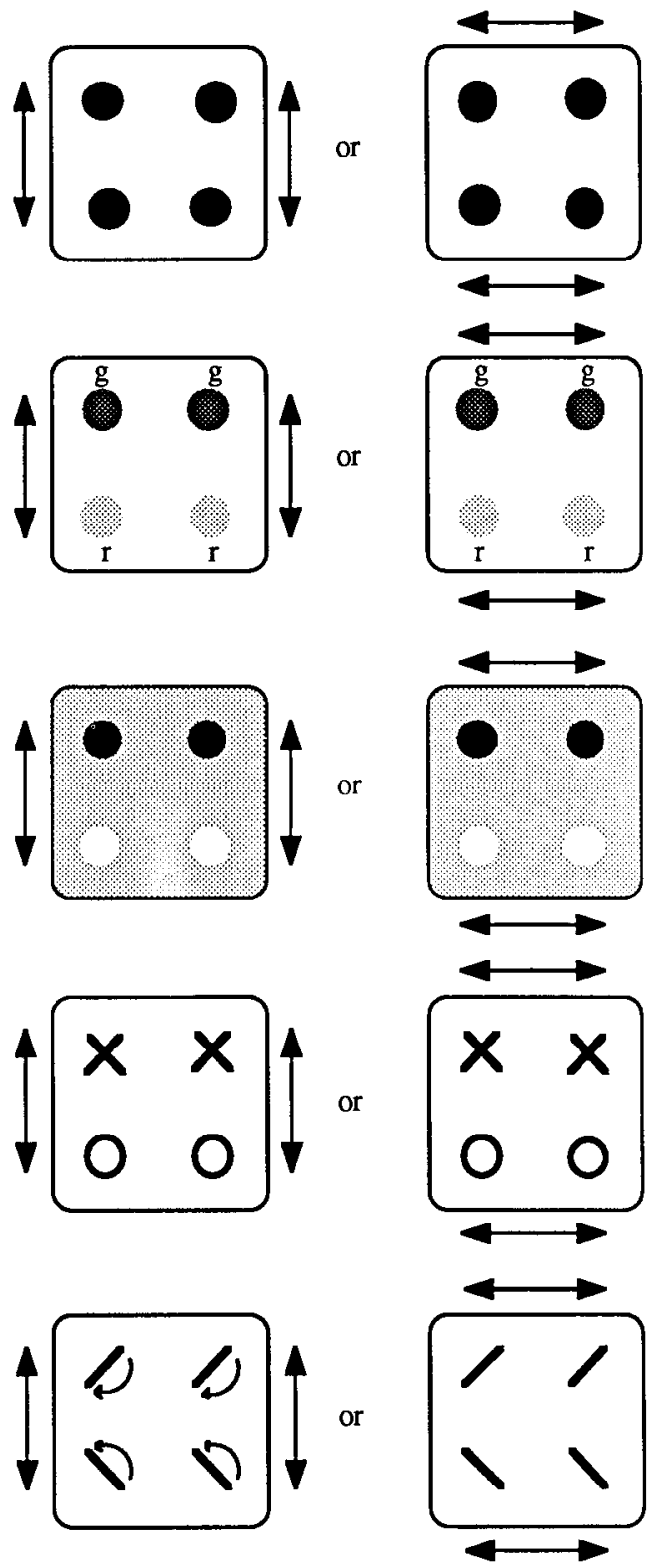

Figure 5. Variations on an ambiguous bistable apparent-motion display in which 2 stimuli alternate between diagonally opposite corners of a square. Movement is seen either vertically (which is slightly preferred) or horizontally, and one's perception tends to flip from one direction to the other spontaneously, or, for low alternation rates, at will (Gengerelli, 1948; Ramachandran and Anstis, 1985). The figure illustrates what was perceived when, instead of the identical spots diagrammed in the top row, we substituted 2 red and 2 green spots (second row), 2 black and 2 white spots, $2 \times s$ and 2 Os, and 2 pairs of lines of differing orientations. None of these substitutions had any obvious biasing effect on the tendency to perceive vertical versus horizontal movement - not enough to overcome the small bias in favor of vertical movement.

perceived motion. When 2 of the dots were white and 2 were black, just as with the red and green dots, we saw no preference for white $\leftrightarrow$ white and black $\leftrightarrow$ black apparent motion over white $\leftrightarrow$ black motion (Fig. 5, third row). Anstis and Mather (1985) have obtained similar results. With white and black dots on a gray background, even though the contrasts of the white and the black with the background are opposite in sign, the contrast of both with the background is nevertheless high. Only when one pair of dots had high luminance contrast $(>20 \%)$ with the background and the other pair less than $5 \%$ was movement between like dots favored. In this situation, the apparent movement of the low-contrast dots seemed slower than that of the high-contrast dots, suggesting a breaking down of the apparent movement itself, rather than a preference for movement between similar or same-sign contrasts.

To examine our ability to detect apparent movement at low contrast, we used the display shown in Figure $6 a$, in which a square was displaced randomly vertically or horizontally, and the subject had to say which. As shown in Figure $6 b$, the smaller the movement to be detected, the higher the contrast threshold. 


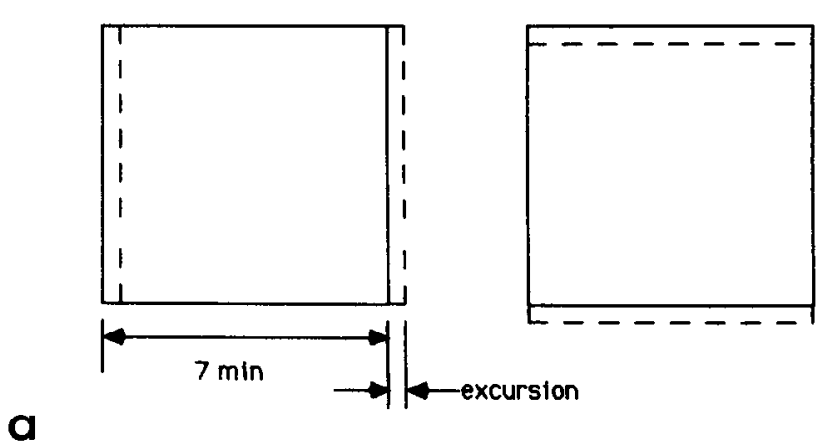

\section{MOVEMENT}

Figure 6. Ability to detect movement as a function of luminance contrast. $a$, The subject was instructed to look at the square, which was shifted in position $1 \mathrm{sec}$ after a warning auditory signal. The shift was horizontal or vertical, at random, and the subject had to say which. $b$, Percentage correct is plotted against luminance contrast for 4 sizes of excursions. A $7 \mathrm{sec}$ excursion could not be detected, and, for larger excursions, thresholds were below $10 \%$ contrast, with little or no improvement above $20 \%$ contrast.

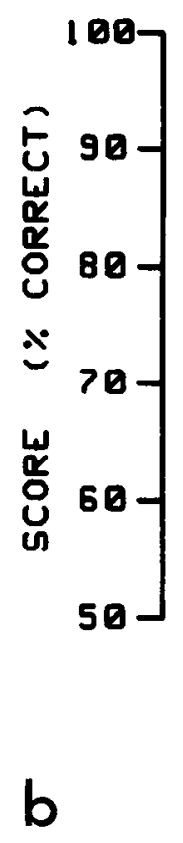

At 10\% luminance contrast, we could detect movements of 15 arc min reliably, and movements of 1.5 arc min at better than chance. Movements of 14 arc sec, in the hyperacuity rangc, required slightly higher contrasts, but beyond $20 \%$ contrast the improvement saturated. These results are similar to the contrast-sensitivity ranges found by Campbell and Maffei (1981) and Thompson (1982) for real movement.

As we will describe, this result is similar to our results for stereopsis, but quite different from the results for form discrimination, in which performance continued to improve above $50 \%$ contrast. The high sensitivity of motion detection to luminance contrast and its insensitivity to color contrast are consistent with its being detected by the magno system alone.

We conclude that some luminance contrast is important, and in certain situations essential, for movement perception. Furthermore, though at low contrasts the amount of luminance contrast influences the perception of apparent movement, the sign of the contrast does not.

We also tested the relative importance of color and luminance contrast on the cooperative linking of movement in different parts of the visual field. Ramachandran and Anstis (1983, 1985; see also Chang and Julesz, 1984), using a display consisting of an array of many bistable apparent-motion squares, found that the perceived directions of apparent movement in different parts of the visual field are strongly correlated - that is, the dots in all the squares move vertically or horizontally, and whenever one square switches perceived direction they all switch simultaneously, like Rockettes (Fig. 7). The squares still appear synchronized, even when some are white and some black on a gray background. But using green dots on a red background, we could find a range of red-to-green brightness over which the synchronization completely disappeared, and each square appeared to move independently (Fig. 7, third row). At times one could even see a spot jumping from one square over to a neighboring square. The abrupt transition from 9 perfectly synchronized little squares to random movement all over the screen, like flying insects, was very striking. The red-to-green ratio for this breakdown was, like the situation with simple apparent movement, consistently about $20 \%$ lower than that for minimizing flicker. The range of $\mathrm{red} / \mathrm{green}$ over which the linking was lost was a bit broader than that for the loss of apparent movement, and even when the synchronization was lost, the apparent movement of the dots did not disappear throughout the screen.

We also asked whether, if the spots in only one square were made equiluminant with the background and the rest of the dots nonequiluminant, the linking between that square and the rest would break down. It did.

With gray dots on a gray background, the movement between 
Display

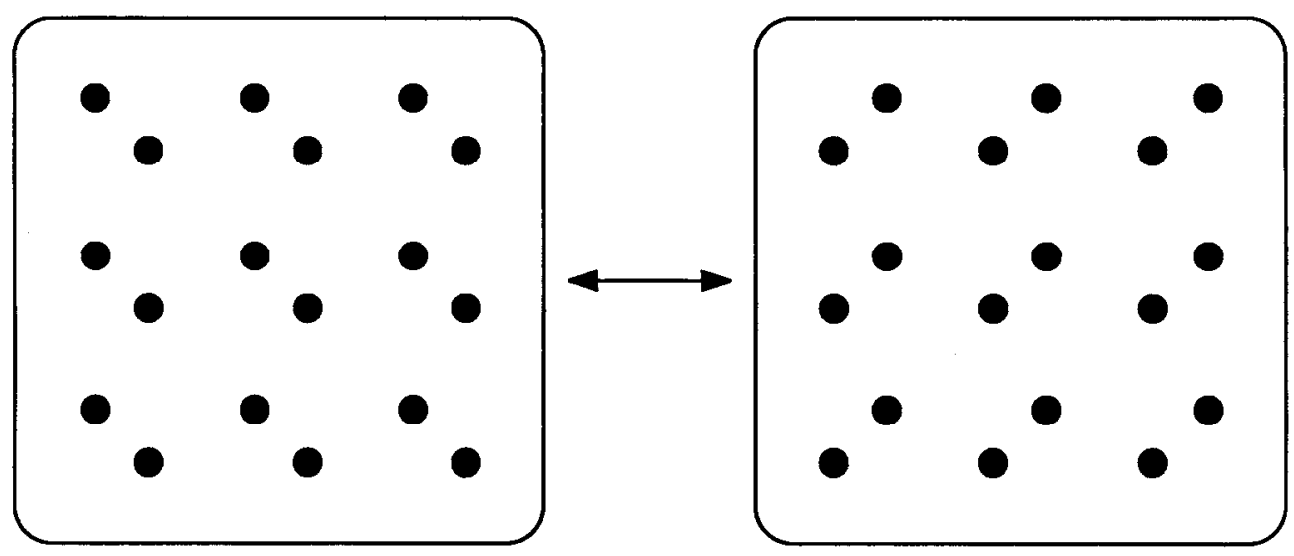

Percept at non-isoluminance
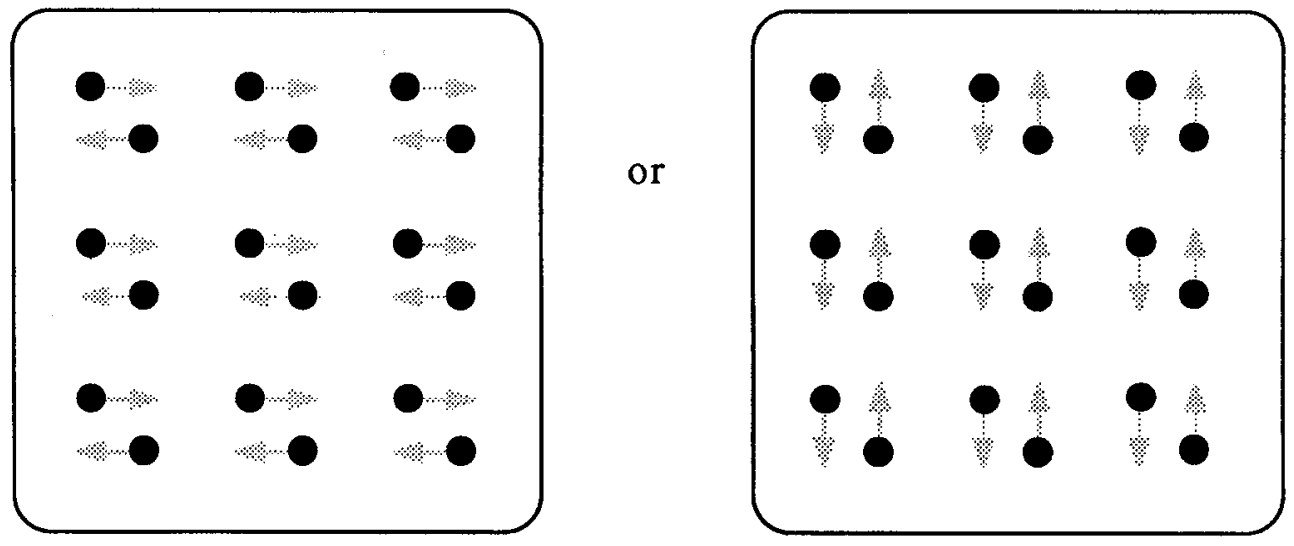

Percept at isoluminance

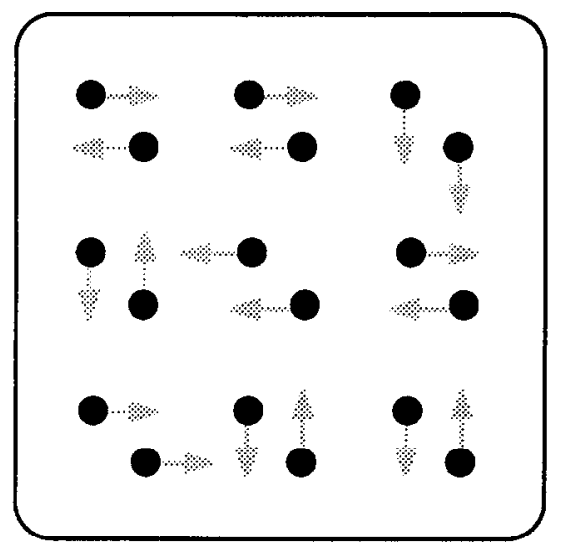

Figure 7. Multiple bistable apparentmotion squares in black and white are perceived as moving synchronously throughout the visual field, as illustrated in upper 2 rows (Ramachandran and Anstis, 1983, 1985). With red spots on a green background, or the reverse, at equiluminance the synchronization broke down, and the apparent movement in each square flipped between vertical and horizontal autonomously, independent of the other squares; occasionally a spot seemed to jump to another square.

squares was synchronized at contrasts of $10 \%$. Below $10 \%$ we had the same sensation as with equiluminant color contrast: everything was moving every which way. This similarity reinforced our impression that the effect of using equiluminant color contrast was equivalent to reducing luminance contrast; that is, the only useful information from a color-contrast border in this situation is the luminance contrast between the colors and not their hue difference. When we slowed down the rate of alter- nation, we found synchronization down to $5 \%$ contrast; below this contrast level, we did not see any apparent movement, just single isolated dots turning on and off, as with colored dots on an equiluminant background.

Another motion phenomenon that disappears at equiluminance is the MacKay illusion (MacKay, 1957). If you look at a high luminance-contrast grating for a few seconds to a minute, letting your eyes move over the grating, and then look at a 
uniform field, you will have the brief impression of a fine pattern of streaming movement in a direction orthogonal to the original grating: Vertical gratings induce horizontal motion; radial spoke patterns induce movement around concentric circles; bull's-eye patterns induce radial motion. We found that when the inducing grating was red and green, it induced a powerful motion aftereffect, except when the red and the green were equiluminant, and then it produced none. As with the synchronization of the ambiguous apparent-motion squares, to produce the aftereffect required only a small amount of luminance contrast. A weak effect could be evoked with gray gratings of only $5 \%$ contrast. (The appearance of the MacKay aftereffects from a nonequiluminant red-green grating was no different from that produced by a black-white one.)

Furthermore, if we looked at a red/green grating and continuously varied the red/green ratio back and forth through equiluminance, we saw a vivid streaming effect each time the colors passed through equiluminance, as if the stripes were invisible to the movement system at equiluminance. (With black/white gratings, the streaming is seen when the stripes are replaced by a uniform field.)

The existence of movement-dependent color aftereffects (Hepler, 1968; Stromeyer and Mansfield, 1970) and color-dependent movement aftereffects (Favreau et al., 1972; Mayhew and Anstis, 1972; Cavanagh and Favreau, 1985) has been interpreted as showing that pathways for color and movement analysis must convergc. But the time required to produce the effects, often many seconds or minutes, and the duration of many of the effects, up to several days, imply that the convergence of the 2 systems may occur at some advanced stage in visual processing.

\section{Contribution of form information to movement perception}

Here we ask a set of questions about the relationship between form perception and movement perception similar to those just asked for color and movement. From the physiology and anatomy we have hints that there are 2 separate systems, both carrying information about edge orientation, and both very sensitive to stimulus motion. To recapitulate the neurobiological evidence: On one side, favoring the idea of different functions, we have the relative independence of the parvo-interblob-palestripe path, and the magno-4B-thick-stripe-MT path, and the suggestions from the physiology that the parvo system is involved somehow with form discrimination, and the magno with movement, stereopsis, and some form information. On the other side, any idea of a strict segregation of form and movement perception must be qualified, since the physiological properties of cells in both pathways suggest a less clean separation than is found for color versus movement. At the cortical stages of the magno system, cells are selective for movement or stereopsis, but they also are selective for stimulus orientation. Furthermore, the orientation information in the 2 pathways does not appear to be completely independent, since not only are the retinotopic maps in layers $4 \mathrm{C} \alpha$ and $4 C \beta$ in precise register, but the orientation preference columns in the 2 systems are also probably in register (Hubel and Wiesel, 1968, 1974; Tootell et al., 1982). Movement seems to be important in the parvo pathway, as well as in the magno pathway: cells in the interblobs of 17 and the pale stripes of 18 almost always respond better to moving stimuli than to stationary ones. Nevertheless, form can be seen in a tachistoscopic flash, and therefore does not require movement; and it could be argued that movement sensitivity of cells in a form system might be important for overcoming adaptation and might not be involved in movement perception at all. Indeed, it is known that when an image is stabilized on the retina, its borders disappear (Troxler, 1804; Adrian, 1928; Ditchburn and Ginsbørg, 1952; Riggs et al., 1953). While a similar argument could be made against the magno system's being involved in movement, the case there seems stronger because of the special qualities of the cells - the prevalence in MT and layer $4 \mathrm{~B}$ of area 17 of directional selectivity, which would hardly be necessary for overcoming adaptation, and the sensitivity of cells in MT to differential movement of stimulus and background (Dubner and Zeki, 1971; Dow, 1974; Maunsell and Van Essen, 1983a; Allman et al., 1985a, b).

What can psychophysics tell us about functional differences between the 2 systems? One way of discriminating psychophysically the type of form and movement information carried by the parvo system from that carried by the magno system is to take advantage of the fact that the magno system has a 2-4fold lower spatial resolution, at a given eccentricity, than the parvocellular sytem. This is so in the retina, the geniculate, and in layer 4C of area 17 (Blasdel and Fitzpatrick, 1984; Derrington and Lennie, 1984; Perry et al., 1984). To examine psychophysically the influence of fineness of pattern on movement, Campbell and Maffei (1981) put gratings of different spatial frequencies on slowly rotating $(1 \mathrm{rpm})$ disks and found that high spatial-frequency gratings ( 16 and 32 cycles $/ \mathrm{deg}$ ), or high spatialfrequency dot patterns, though clearly resolvable as gratings or dots, were perceived as rotating much more slowly than lower spatial-frequency patterns, and often seemed to be entirely stationary. This was so despite the observer's ability to deduce the movement, in the case of gratings, from the change in the grating's angle. Campbell and Maffei (1981) called this phenomenon "stopped motion." We reproduced their demonstration for ourselves and found the effect dramatic. The illusory stoppage of motion was similar to that seen with equiluminant colored stripes. We will describe later the similar loss of stereopsis with high spatial-frequency patterns.

Further evidence that movement perception and some aspects of form perception are independent comes from threshold and cross-adaptation studies. For a moving or flickering sine-wave grating, the contrast threshold for detecting the flicker or movement and the threshold for resolving the grating are different, and these 2 thresholds vary independently with spatial frequency (Van Nes et al., 1967; Keesey, 1972; Kulikowski and Tolhurst, 1973). At low spatial frequencies, movement or flicker can be detected at lower contrasts than those needed to resolve the grating; with high spatial frequencies, the form can be resolved at lower contrasts. With low and middle spatial-frequency gratings, flicker in which alternate frames differ in phase by $180^{\circ}$ produces apparent movement, but with a high spatialfrequency grating the form can be resolved at lower contrasts than the flicker, and the grating then looks stationary (Kulikowski, 1971); this may be similar to the "stopped motion" of Campbell and Maffei (1981). Tolhurst (1973) found that preadaptation with a moving grating reduced the sensitivity for detecting either a moving grating or a stationary grating, but preadaptation with a stationary grating affected the sensitivity for a stationary grating but not a moving one. All of this suggests that there is a form channel that is sensitive to motion, and, in addition, a motion channel that is independent of the form channel.

Another test for the independence of form and movement is 
to examine the influence of form on apparent movement, as was done in the previous section for color versus movement. Kolers and Pomerantz (1971) tested the effects of shape on the threshold for experiencing apparent movement, and, as in the case of color, they found that the form or shape of the alternating stimuli had little effect. We used the bistable apparent-motion square described above as a potentially more sensitive test for any influence of form on apparent movement. Making the stimuli different in shape, say two $\times s$ and two Os, had no obvious influence on direction preference (Fig. 5, fourth row). We also tested the effect of using lines of different orientation (Fig. 5, fifth row) and found that line orientation had no effect on preferred direction of movement. The observer was just as likely to see movement between orthogonal lines as between parallel ones, and when the movement was seen between lines of unlike orientation, it was accompanied by an apparent rotation. Our findings confirm earlier studies (Kolers and Pomerantz, 1971; Navon, 1976) that form, at least the fine detail of shape, has little influence on the early stages of the movement-detection system. Green (1986), using a different type of stimulus, has, however, found a weak influence of orientation on the perception of apparent movement.

All of these results suggest that some components of form and movement are analyzed independently by the visual system.

\section{Stereopsis}

In a 3-dimensional scene, the patterns of light projected on the 2 retinas are different, and the differences are interpreted as information about the relative distances of objects from the observer (Wheatstone, 1838). To interpret the differences correctly, the brain must determine which points in the 2 retinas correspond to the same point in the scene. Correct point-topoint matching based solely on luminosity at each point would require a formidable calculation in a complex scene, since ambiguities would arise as a result of many points' having similar or identical luminances. The problem would be greatly simplified if the system could use additional information at every point, such as edge orientation, movement, or color.

A simple example of the problem of stereomatching is illustrated in Figure 8 . When the frames in the 2 diagrams shown in the top row are fused as indicated, the images of the dot fall on noncorresponding points in the 2 retinas, and the observer sees the dot at a depth different from that of the frame. In this example, the dot appears to lie in front of the frame. When the 2 images of the second row are fused, a single dot is seen at a depth behind the frame. If the images in the 2 rows are now combined, as in the third row, the resulting percept is not the combination of the first 2: Two dots are seen side by side in the plane of the frame; almost never is one dot seen in front of the frame and one behind it, even though that would seem logically possible. Similarly, for the pair of images shown in the fourth example (Marr and Poggio, 1976), the observer sees only 4 dots lying in the plane of the frame, rather than any of the 12 other possibilities arranged like bowling pins in front of or behind the frame. In these examples, color and orientation obviously do not help in the matching, since the color and shape of the objects are identical. But the fact that our brains come up with a single preferred solution despite the possibility of other equally correct solutions does not necessarily mean that other cues, if available, such as color, movement, or form would not be used to confirm or override the decision. From what we know about the physiology of stereotuned cells, we would guess that edge orientation and movement are used, but not color. Here we ask what evidence psychophysics can bring to bear on the question.

Contribution of color-contrast and luminance-contrast information to stereopsis

Anaglyphs. Several lines of psychophysical evidence support the conclusion that color is not used in solving the problem of stereomatching.

A convenient type of stereogram is called the anaglyph (Rollmann, 1853; Helmholtz, 1910; Julesz, 1971): one pattern, made of red and white dots, is viewed by one eye through a green filter (giving a black-and-green pattern), and the other pattern, of green-and-white dots, is viewed with the other eye through a red filter (giving a black-and-red pattern). The 2 patterns can be superimposed, avoiding the need to cross or diverge one's eyes or use a stereoscope or polarizers. Since one can see depth in such stereograms, it has been argued (Ramachandran and Sriram, 1972) that color must not be very important in stereopsis: if it were, using different colors in the 2 eyes should lead to rivalry and so interfere with the process. By itself, this argument might seem weakened by the fact that when an entire visual field is viewed through a colored filter, the color is seen as very desaturated (Land, 1959; Lettvin, 1967; Julesz, 1971), so that each eye sees essentially a luminance-contrast scene, not a colored scene. We therefore asked whether one can still experience stereopsis when strong rivalry due to color-mismatching actually exists. We viewed a red/green random-dot anaglyph (Julesz, 1971) through pieces of red and green Wratten filters, each just large enough to cover the anaglyph for one eye when held at arm's length (Fig. 9), with the rest of the visual field occupied by the laboratory in its usual colorful mess, well illuminated by ordinary white tungsten light. The small region of the visual field covered by each filter was, as expected, vividly colored, red for one eye and green for the other; within this region of overlap, retinal rivalry was marked, the apparent color shifting from red to green with a lustrous quality characteristic of retinal rivalry (Helmholtz, 1910). None of this interfered in the least with a vivid sensation of depth, supporting the idea that color does not play a strong role in stereopsis. (If the 2 image pairs of the anaglyph were replaced with 2 identical printed black-and-white texts, the readability was similarly not at all impaired despite the strong rivalry of the background colors.)

\section{Color as a cue to solving the stereomatching problem}

As another test for the involvement of color in stereopsis, we made a stereopair similar to Figure $8 d$, except that the positionally corresponding dots were of different colors. To do this, we projected through orthogonal polarizers 2 slides of 5 colored dots lying in a row centered in a frame, and viewed the screen through 2 crossed polarizers, so that the right eye saw one image and the left eye the other. In the left-eye image, the sequence of dot colors was yellow, blue, green, red, orange; in the righteye image, the sequence was shifted by one dot to blue, green, red, orange, yellow (or to orange, yellow, blue, green, red). When the 2 images were viewed with the frames aligned, the dots, for most observers, combined so that the 2 farthest to the left fused with each other (a blue dot fused with a yellow), the second dots fused (green with blue), and so on. Each dot appeared to have a shimmering, lustrous surface, with an alternation from onc color to the other. Occasionally the fusion would shift so that like colors fused with like, but this happened less frequently than fusion by position. Thus even though 4 dots could have 
DISPLAY
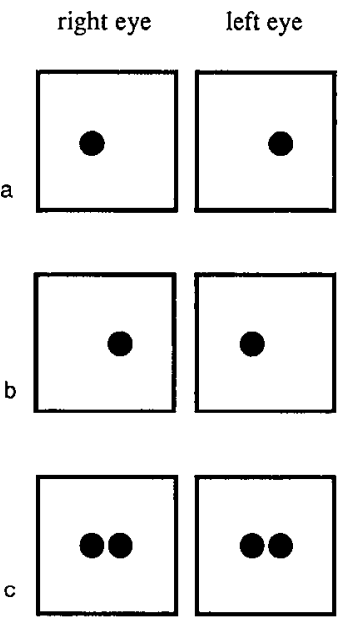

PERCEPT

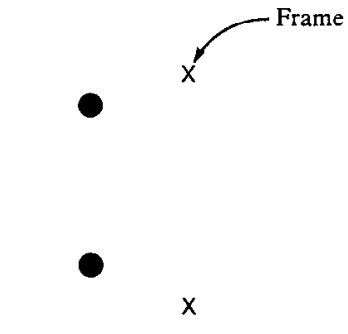

possible percept observed percept

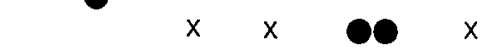

Figure 8. Illustration of the problem the nervous system must solve in stereomatching. DISPLAY shows the image viewed by each eye, and PERCEPT what is perceived. $\times s$ Represent the borders of the frames, and the depths of the spots relative to the frame are indicated as if viewed from above. The spots in $a$ appear in front of the frame, and the spots in $b$ behind it; the same spots together, in $c$, are perceived as lying side by side in the plane of the frame, and never in the equally logically plausible conformation, with one in front and the other behind. In $d$ (from Marr and Poggio, 1976), 4 identical squares lying in one plane are seen only as 4 squares at the same depth, never as any of the other 12 possible percepts. fused, like color to like color, had the fusion shifted by one dot, the preferred fusion was simply by position. This result is in sharp distinction to the results for orientation and movement, described below, in which even one misaligned orientation- or movement-specific cue in the presence of 4 identical dots tended to drive the fusion plane of the dots, and 2 or more such cues unambiguously determined the match.

\section{Random-dot stereograms}

A quite different, and more direct, suggestion that color information is not used at all in stereopsis comes from the demonstration by Lu and Fender (1972) that depth perception is difficult or absent in random-dot stereograms composed of 2 equiluminant colors instead of black and white, even though the patterns in the 2 eyes fuse and their details can be clearly discriminated. Since Lu and Fender's (1972) original observa- tion, the contribution of color information to stereopsis has been a subject of considerable dispute. Comerford (1974) failed to find loss of stereoscopic depth at equiluminance for simple figures. Lu and Fender (1972), Gregory (1977), and de Weert (1979) found loss of depth for random-dot stereograms but not for simple figures. de Weert and Sadza (1983) failed to find loss of depth for either figural stimuli or for random-dot stereograms. We naturally wondered why the results should differ for different tasks and from one laboratory to the next. The possibilities that occurred to us included failure to achieve equiluminance because of the use of excessively coarse steps in varying the relative luminance of the 2 colors $(0.04 \log$ unit or $10 \%$ steps, for example, in Comerford's study), variation in equiluminance because of chromatic adaptation from using prolonged stimuli, variation in equiluminance with eccentricity and differences in the degree of this variation from subject to subject, and, finally, 


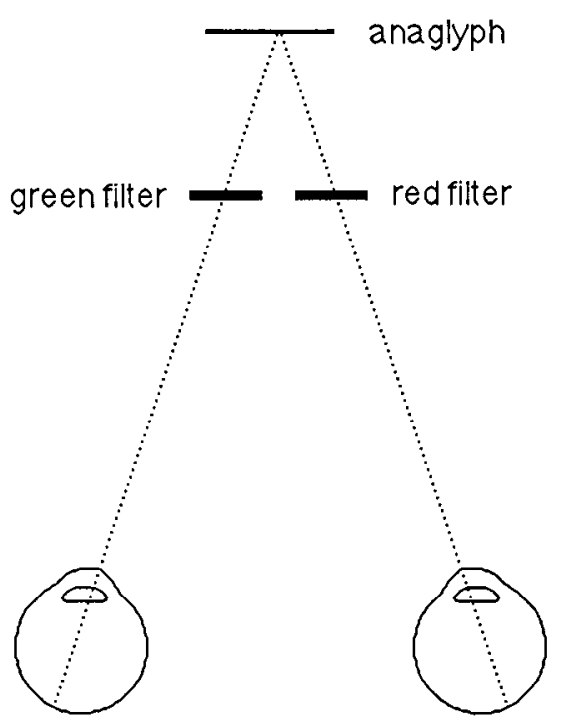

viewer right eye

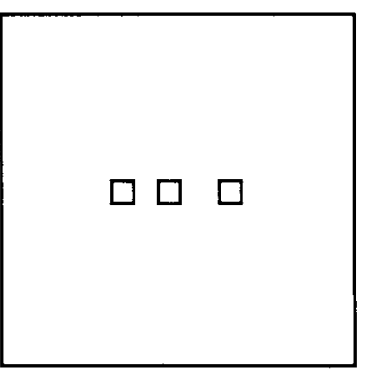

a.

right eye

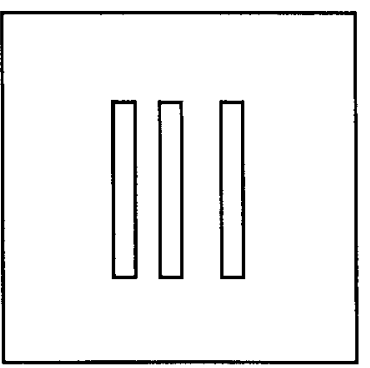

b.

Figure 10. Two stereopair displays used for testing stereopsis at equiluminance. $a$, The display consists of $30.25^{\circ}$ squares, red on a green background or green on a red background, with a 2.5 arc min inward or outward displacement of the center squares relative to the flanking reference squares. With unlimited viewing time, we could find no relative intensities of red and green at which stereopsis failed. But if the squares were presented for only $50 \mathrm{msec}$ (before the presentation the ohserver fixated on a pair of fixation points in the centers of the frames), stereopsis fell to chance levels over a narrow range of relative green/ red intensities (Fig. 11). $b$, Bars were $2^{\circ}$ long and $0.25^{\circ}$ wide and presented for $50 \mathrm{msec}$. As the red/green ratio was varied, some part of the bar always seemed to lie out of the plane of the frames, either obliquely in depth or curving in and out. We attribute this to the variation in equiluminance with eccentricity (see Fig. 3).

for us to imagine why stereopsis of random-dot patterns and simple figures should involve different mechanisms. We first tried looking at a simple figural stereogram, such as the one shown in Figure 10a. The display, which we free-fused at 1.5 $m$ distance, consisted of a green background with a black fixation point, two $0.5^{\circ}$ red reference squares, and a central $0.5^{\circ}$ red square at a different disparity. We also used green squares on a red background, with identical results. The relative luminance ratio of squares to background could be varied continuously. The center square was displaced horizontally by $2.5 \mathrm{~min}$ of arc in each eye, so that it appeared either in front of or behind the other two. At the luminance ratio of red to green at which depth disappeared for random-dot stereograms, we found that we could still see some depth in this simple figure. We wondered if this might be due to some difficulty in achieving equiluminance, and were particularly concerned about eye movements for 2 reasons.

First, with a simple stimulus, either adaptation or the formation
Though stereoscopic depth in figural stimuli has been reported by several groups not to disappear at equiluminance, it was hard 


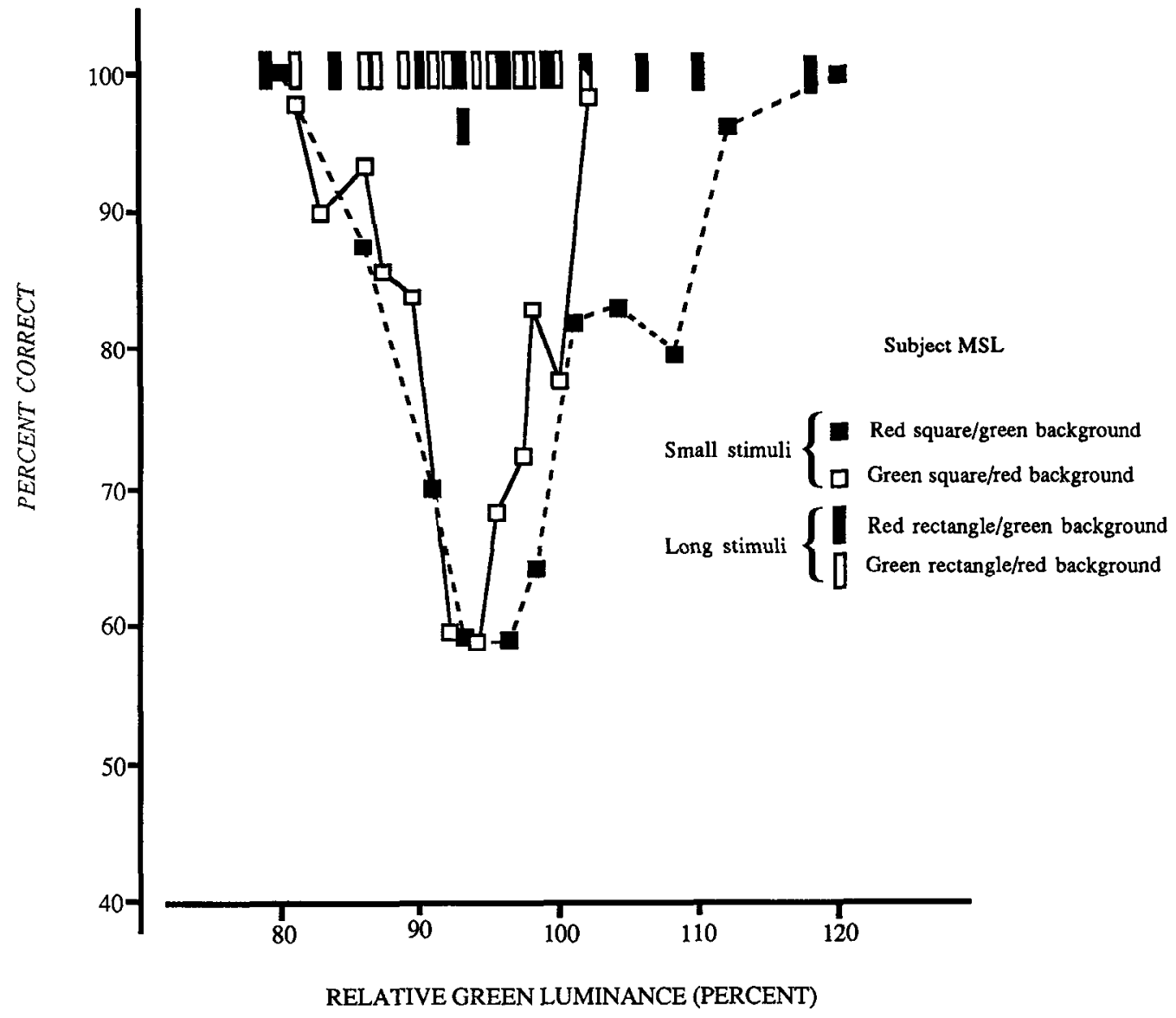

a

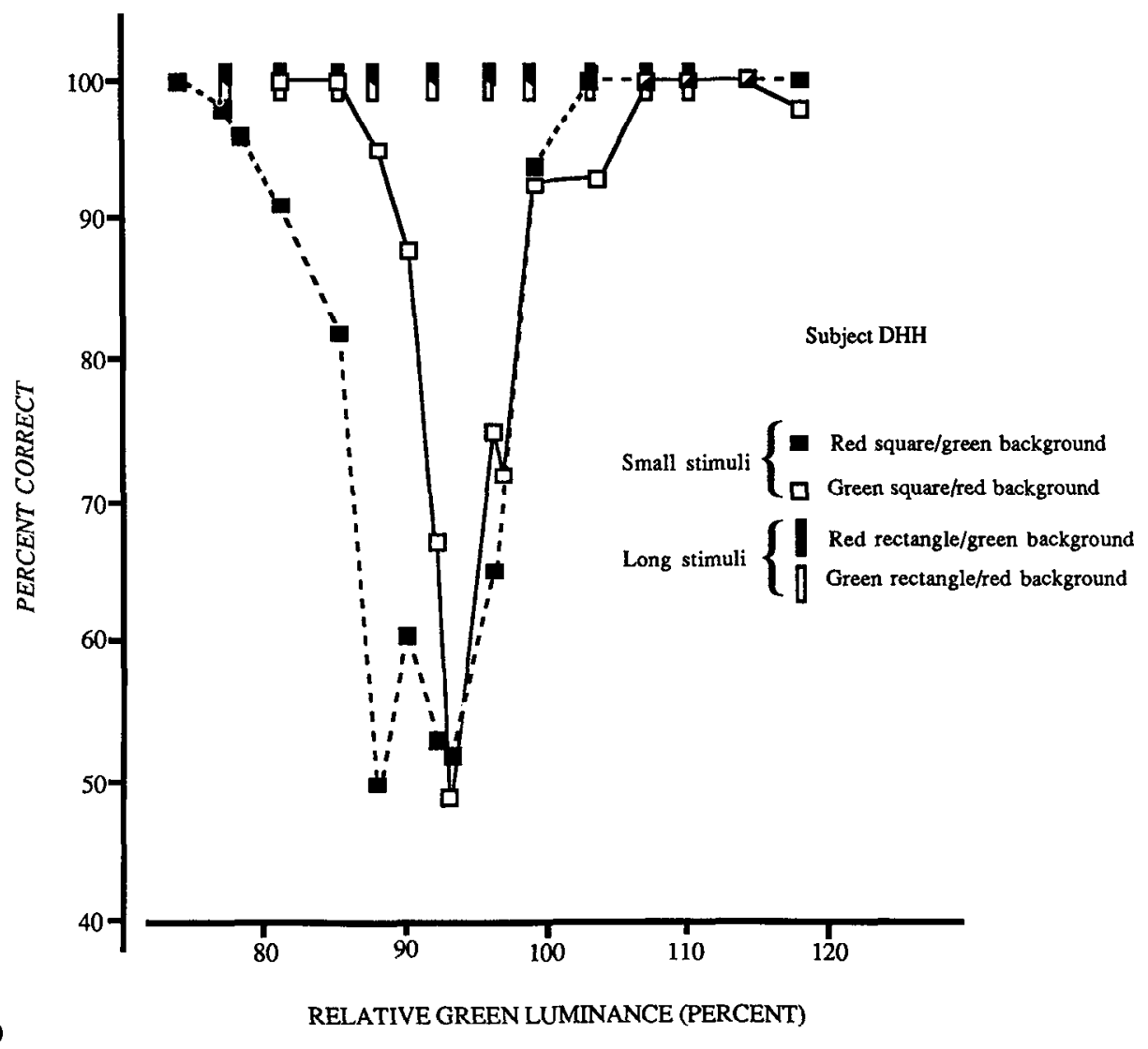




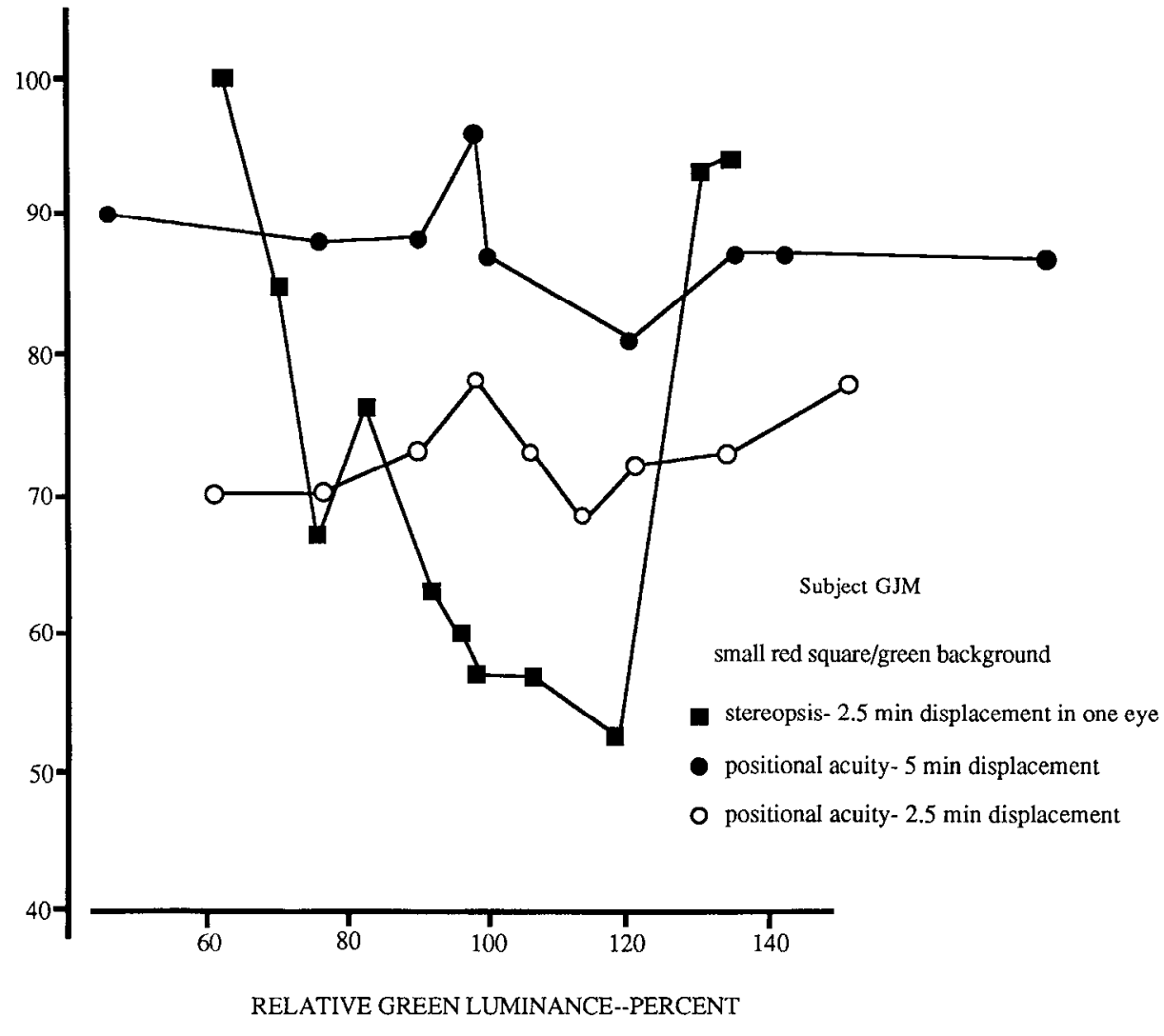

Figure 11. $a-c$, Results of forcedchoice testing in the experiments described for Figure 10 for 3 subjects. The rectangles show the results of testing with $0.25^{\circ} \times 2^{\circ}$ rectangles and 2.5 arc min displaccment in each eye. Stereopsis was possible at all red/green settings. For squares of $0.25^{\circ}$, and the same disparity, stereopsis fell almost to chance levels over a narrow red/green ratio. For subject G.J.M. (c), we reduced the size of the stimulus to $0.125^{\circ}$ and the disparity to 2.5 arc min in one eye only. His performance then fell to chance over a broader range of red to green. We compared this to his ability to discriminate differences in position of 5 and 2.5 arc min of the center square relative to the reference squares viewed binocularly. of afterimages might change the equiluminance point at some retinal positions, and we suspect that this might be more serious with simple stimuli. Second, if the stimuli are large or if the eyes can rove about the image, the variation in equiluminance with retinal eccentricity could become important. We therefore made the squares smaller $\left(0.25^{\circ}\right)$ and shortened the time in which the stimulus was presented to $50 \mathrm{msec}$, with several seconds between presentations.

With this stimulus we found a narrow range of red/green ratios over which neither of us could detect depth at better than chance (Fig. 11, a, b).

In order to learn whether variations in equiluminance with retinal eccentricity were responsible for our initial failure to lose stereopsis at equiluminance, we tested ourselves with longer bars, still presented briefly. With $2^{\circ} \times 0.25^{\circ}$ rectangles, using the same protocol as with the small squares, we could find no setting at which our performance in judging the test bar to be in front or behind was even slightly impaired (Fig. 11, $a, b$ ). With these long bars we had the distinct impression that at equiluminance only part of the central bar was in a different plane of depth from the other 2 bars: it either appeared to lie obliquely in depth or to be curved in depth. Slight changes in the ratio of red to green made different parts of the bar bulge out or in. This result suggests that equiluminance variation across the visual field is an important problem in assessing the contribution of color to stereopsis in a large figure or with the eyes free to move.

Figure $11 c$ shows the performance of a third subject (G.J.M.), which did not decline to chance levels until we reduced the width of the stimulus to $0.125^{\circ}$, reduced the displacement to
2.5 arc min in only one eye relative to the other, and randomized which eye's image was displaced. Subjects can detect stereoscopic depth with retinal disparities much smaller than the minimum displacement detectable nonstereoscopically (Westheimer and McKee, 1979), so we compared his positional acuity by using a single image viewed binocularly and displacing the central square relative to the reference squares. As shown, unlike stereopsis, his positional acuity did not show any minimum at equiluminance, even when the displacement was only $2.5 \mathrm{~min}$.

From these results we conclude that the capacity for seeing stereoscopic depth does deteriorate at some ratio of green/red brightness, and this ratio varies markedly from subject to subject over at least a 2 -fold range.

Table 4. Ability to see stereoscopic depth in equiluminant randomdot stereograms

\begin{tabular}{lll} 
Subject & $\begin{array}{l}\text { Lreen/red } \\
\text { (\% correct) }\end{array}$ & $\begin{array}{l}\text { Setting } \\
\text { (normalized } \\
\text { to subject } \\
\text { D.H.H.) }\end{array}$ \\
\hline M.L. & 52 & 0.7 \\
D.H. & 50 & 1 \\
D.C. & 60 & 1.0 \\
J.R. & 60 & 1.2 \\
O.B. & 58 & 1.2 \\
P.G. & 55 & 0.9 \\
\hline
\end{tabular}



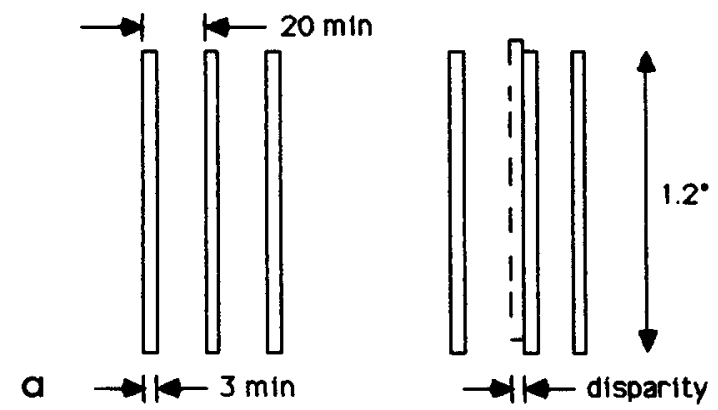

Figure 12. a, Display for testing stereopsis at low luminance contrasts. In each member of the stereopair, the 2 outer reference bars were high luminance contrast, solid white on a darker background, and were present continuously. A center gray bar, of variable contrast with the background, appeared for $0.5 \mathrm{sec}$. In one eye it was centered between the 2 reference bars; in the other, randomly the left or the right eye, it was displaced from the center by a variable distance to the left or right, at random. For each test the subject had to say whether the center bar was behind or in front. $b$, Results of forced-choice testing. Performance is plotted against percentage contrast for 4 different displacements. As in the case of movement, for the 3 larger disparities performance was better than chance at less than $10 \%$ contrast and improved as contrast increased up to about $20 \%$, and then leveled off. For the smallest disparity tested, stereopsis failed at all contrasts.

\section{STEREOSCOPY}

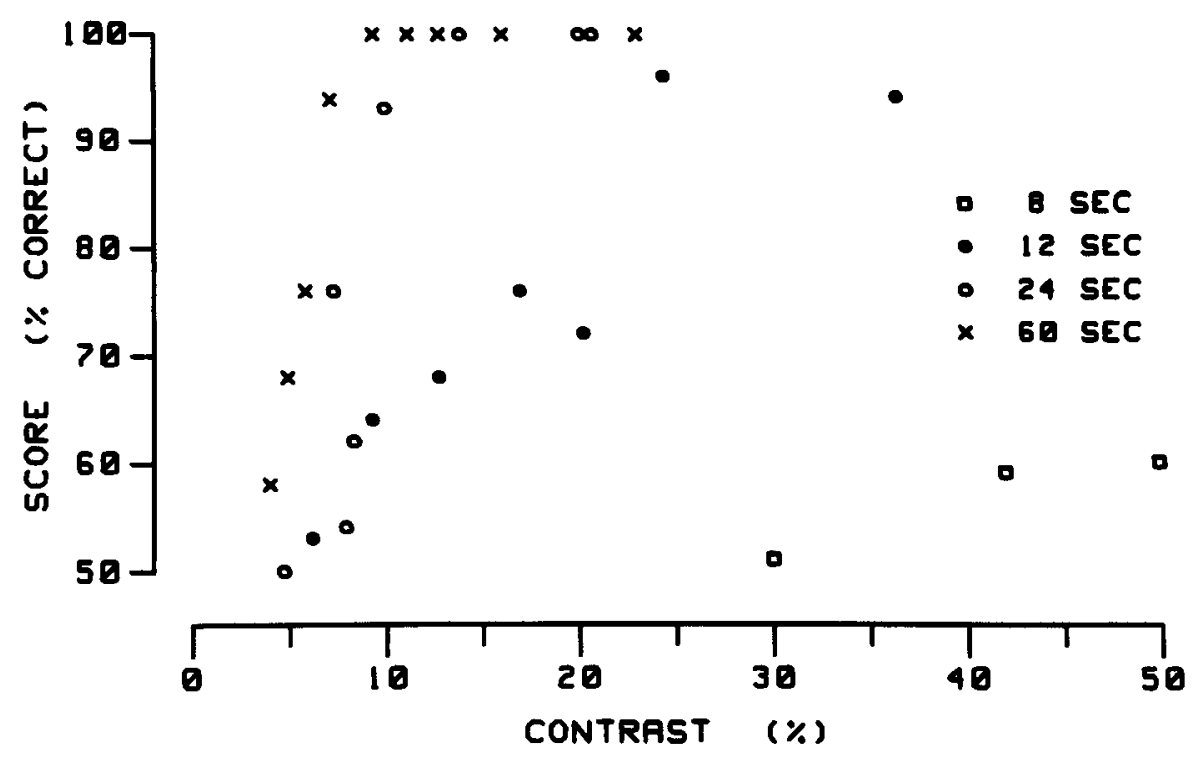

\section{Stereopsis at low luminance contrast}

We tested the contrast sensitivity of the stereopsis system by generating random-dot stereograms in which light and dark dots differed in brightness by very small amounts. The lowest luminance contrast at which we could clearly detect depth was $5 \%$. At this contrast we had a clear sensation of depth, though when we looked with both eyes at either member of the stereopair, we could not discern the individual dots. The fact that at $5 \%$ luminance contrast we could see depth but had lost high resolution form perception is consistent with the possibility that the stereo system gets information primarily from the magnocellular pathway.

We tested stereo acuity at various levels of luminance contrast, using a forced-choice protocol in which the subject reported whether a central bar was in front of or behind 2 flanking bars (Fig. 12a). As shown in Figure 12b, some stereopsis was present at very low levels of luminance contrast, and performance improved with higher contrasts, but only up to about $20 \%$. At $10 \%$ contrast, the stereopsis was better than chance for disparities of 12 arc sec, a performance that approached hyperacuity.

\section{The Pulfrich phenomenon}

Pulfrich (1922) found that if a pendulum bob is swung across the visual field and viewed with a neutral-density filter over one eye, the path of the bob appears to be elliptical in depth, coming toward the observer and then moving away as it swings back and forth. The illusory depth occurs because decreasing the amount of light to one eye delays the processing of input from that eye (Julesz and White, 1969), and a delay in one eye produces disparity, since the bob is moving. We generated a video display (Fig. 13) consisting of 2 stationary red reference squares placed above and below a central red square that moved back and forth horizontally, against a green background. When the red and green were not equiluminant, putting a $1 \mathrm{log}$ unit neutral-density filter over one eye caused the central square to appear alternately behind and in front of the screen by about 1 $\mathrm{cm}$ as it moved back and forth. When we adjusted the relative brightnesses of the red and green, near equiluminance the apparent depth became less and less, and over a narrow range all sensation of movement in depth was lost. This result is consistent with the loss of stationary stereopsis at equiluminance and further supports the notion that the stereopsis system ignores color contrast, measuring the retinal disparity of only luminance-contrast borders.

\section{Form information in stereopsis}

In asking about the relationship between form perception and stereopsis, we encounter problems similar to those in discussing form and movement. The anatomy and physiology suggest that there should be a separation between stereopsis, which seems to be carried predominantly by the magno system, and those aspects of form perception carried by the parvo system. The 


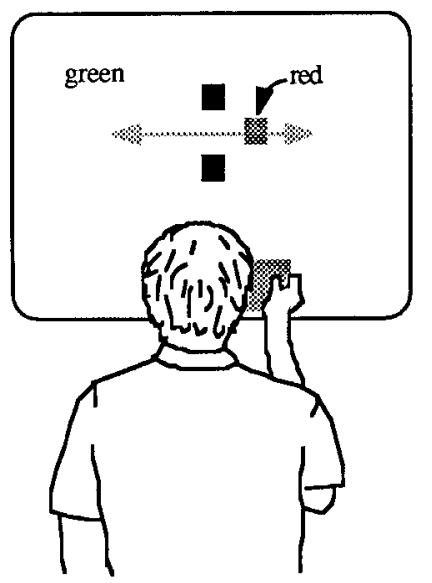

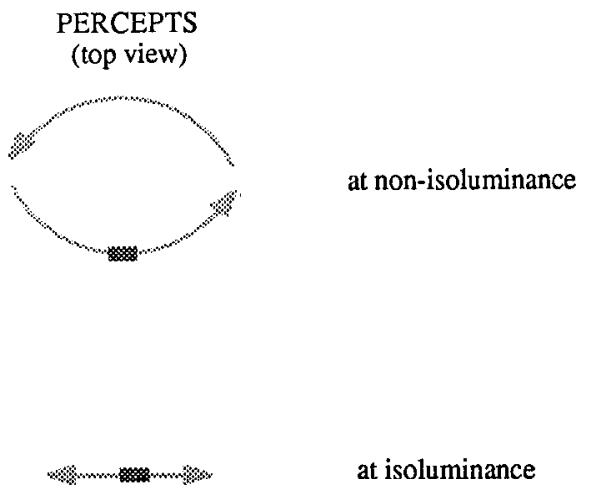

at isoluminance
Figure 13. Procedure for testing the Pulfrich phenomenon at equiluminance. A red square moved back and forth horizontally between 2 stationary red squares on a variable-luminance green background. (The squares were $1 / 2^{\circ} \times 1 / 2^{\circ}$, and the total excursion was $4^{\circ}$. Rate of movement was $1 \%$ sec.) The display was viewed with both eyes, one of which was covered by a $1 \log$ unit neutral-density filter. Away from equiluminance, the middle square appeared to move elliptically in front of, then behind the plane of the screen, but over a narrow range of red/green intensities, all sensation of depth disappeared. orientation selectivity of stereotuned cells could be interpreted as playing some role in form perception, but it could also be used to help resolve ambiguities in stereomatching. We looked for evidence to justify the idea that in stereopsis some information about form or edges is used in solving the problem of matching or correlating images, but that this form information is carried by a different system, segregated from the system whose major function is the analysis of form.

Though physiological studies in animals make it clear that cells selective for retinal disparity are also orientation-selective (Hubel and Wiesel, 1962, 1965, 1970; Barlow et al., 1967; Pettigrew et al., 1968; Bishop et al., 1971; Clarke et al., 1976; Poggio and Fischer, 1977), discussions of possible models for stereopsis mechanisms have often seemed to favor, or have at least concentrated on, the possibility of a point-to-point matching without the use of edge-orientation information. The justification for this approach seems to be an implied assumption that random-dot patterns lack orientation-specific cues, and that the perception of depth in random-dot stereograms must therefore rely solely on point-to-point matching (Julesz, 1971). But a pattern of random dots contains a rich assortment of dot clusters whose borders furnish distinct and recognizable contours. To be sure, random-dot patterns with widely spaced dots, and conscquently with little obvious clumping, can also generate a strong sensation of depth (Julesz, 1971), but orientation-specific cells would, in that case, presumably respond to the contours of the individual dots or to doublets produced by nearest-neighbor dots.

Some theoretical formulations of stereopsis, notably those of Marr and Poggio (1976, 1979) and Poggio and Poggio (1984), have emphasized the importance of borders in stereomatching, generally modeled in terms of "zero crossings," using cells with circularly symmetric receptive fields, such as are found in retinal ganglion cells, geniculate cells, and cells of layer 4 of area 17, all of which are strictly monocular. While we agree that borders are what are likely to be matched in stereopsis, we prefer to discuss the theory in terms of the known physiological properties, including orientation tuning, of cells that do receive input from the 2 eyes, preferably of cells with disparity tuning. The physiological observation that stereotuned cells are usually also orientation-tuned does not, of course, prove that orientation information is used in stereomatching, but the presence of orientation selectivity at the first stage at which information from the 2 eyes converges does seem to support the idea. The psychophysical evidence (Julesz, 1960, 1971; Mayhew and Frisby,
1978) seems not, to us, to weigh heavily against the use of orientation information in the stereomatching mechanism, but we could not find much direct support for the idea either. We therefore tried to examine the extent to which the stereomatching process is influenced by the presence of orientation information in the stimuli.

To test the influence of orientation information on stereomatching, we used the patterns shown in Figure 14. When we substituted an oriented line for one of the dots in the left-eye pattern and for a different, adjacent dot in the right-eye pattern (Fig. 14, second row), the 2 lines showed a marked tendency to fuse with each other rather than with the 2 positionally corresponding dots. Occasionally, but much less often, position would prevail and the observer would see each line fused with a dot. When the lines fused with each other, they appeared to lie in front of the plane of the frame. Usually the 2 dots on either side of the line also appeared in front of the frame, although occasionally they appeared to lie in the plane of the frame. Thus a single oriented line can determine the fusion plane not only for itself, but also for nearby dots. The unmatched dots at the ends appeared metastable or blurred, as is typical in situations of retinal rivalry. Two or more oriented lines in each image formed an even stronger stimulus for driving fusion out of the plane of the screen, since, for all our observers, the lines always fused with each other rather than with the positionally corresponding dots (row 3), and the dots appeared to lie in the plane determined by the lines rather than in the plane of the screen.

Since the effect of oriented lines on stereomatching might be explained in terms of matching individual points, we made the display shown in Figure 15 to test the power of point-to-point brightness correspondence to drive fusion. In this figure, if luminance matching is very important, the thick lines should fuse with each other and the thin lines with each other, resulting in an impression of a thick line lying in front of a thin one. Instead, we found that the thick line in the right-eye image fused with the thin line in the left-eye image and vice versa, so that we saw 2 odd-looking (rivalrous) lines in the plane of the frame. These results suggest to us that orientation of edges may be a stronger cue than local brightness for determining stereomatches.

\section{Movement as a cue for stereomatching}

Having looked at both form and color as possible cues to stereomatching, we decided to try movement. To test movement, we used the stereopairs shown in Figure 16. In each picture, one of the lines in one eye's image and the next line in the other 


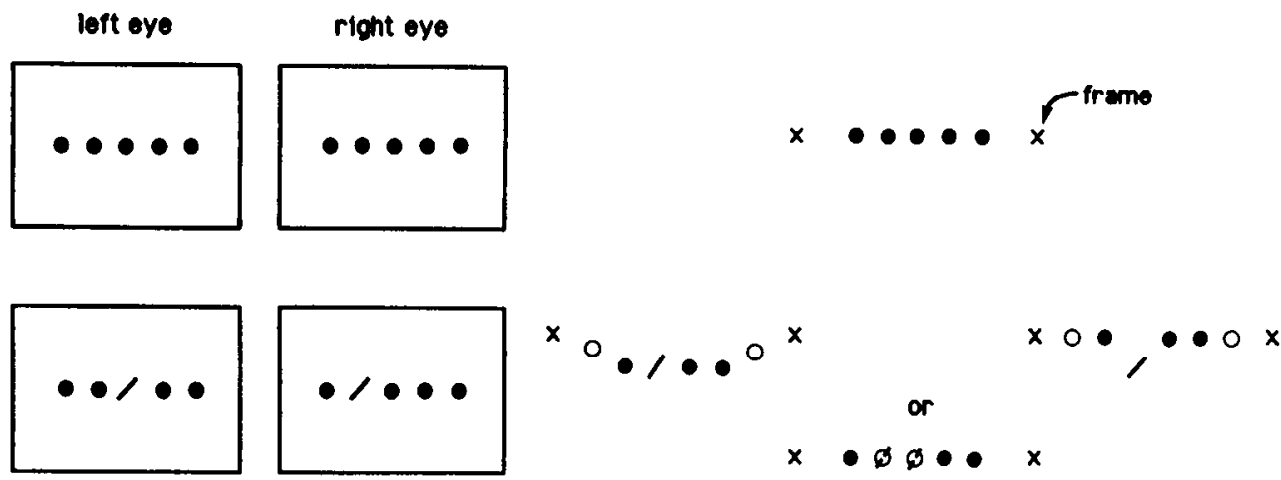

Figure 14. Experiment to test the influence of orientation information on stereomatching. Each display represents 2 figures fused stereoscopically. Each percept represents what was seen, as if the displays were seen from above; down represents towards the observer. Subjects usually matched the oriented lines, fusing elements of like orientation rather than elements of corresponding position. Frequently adjacent dots were captured into the same depth plane as the lines.
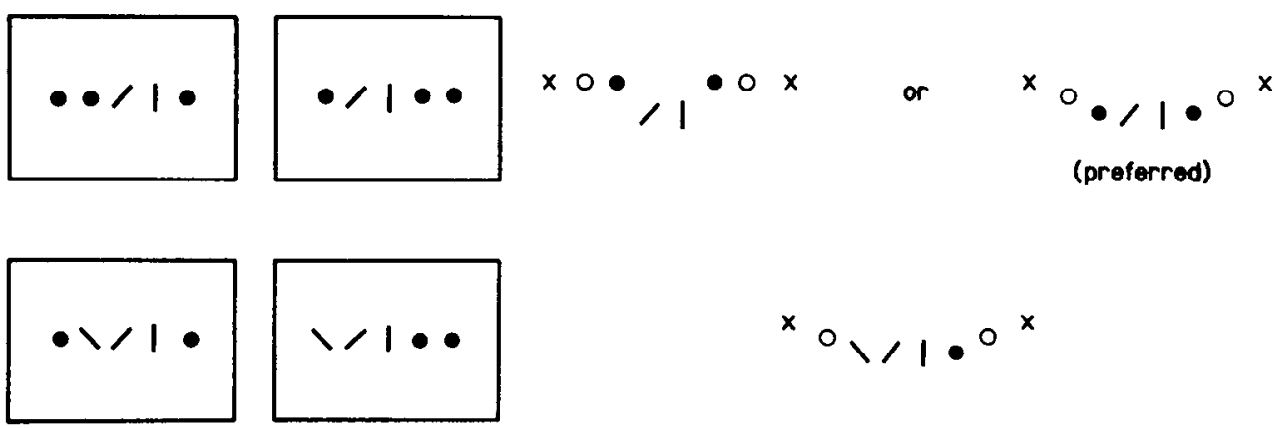

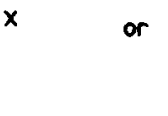

(preferred) eye's image shifted synchronously up and down as indicated, twice per second. The 2 pictures fused so that the 2 moving lines, rather than the 2 positionally corresponding lines, were matched, and appeared to lie in front of the plane of the frame, despite the resulting rivalry at the ends. For determining stereomatches, apparent movement seemed to have about the same power as orientation, and much more power than color.

\section{Loss of stereopsis at high spatial frequencies}

Since stereopsis shares many magnocellular-like properties with movement, one might expect that it, too, might have a slightly lower spatial resolution than form perception; that is, it would fail with stimuli of high, but resolvable, spatial frequency. To explore the spatial resolution of stereopsis we made the stereograms shown in Figure 17. In the stereogram shown in Figure $17 a$, the grating texture of the background could be resolved at viewing distances of less than $2 \mathrm{~m}$, but at viewing distances farther than $2 \mathrm{~m}$, when the spatial frequency of the grating exceeded $30 \mathrm{cycles} / \mathrm{deg}$, the background was seen only as a uniform gray. At all viewing distances a sensation of depth was experienced, with the small white square appearing to lie in front of a larger background square, as expected. Figure $17 b$ differs from Figure $17 a$ in that it contains very little low spatialfrequency information, and at viewing distances greater than 2 $\mathrm{m}$ (spatial frequency of the grating greater than $30 \mathrm{cycles} / \mathrm{deg}$ ) we could not resolve the stripe pattern in either the central square or the background and saw only a uniform gray. At closer than about $0.5 \mathrm{~m}$ (spatial frequency of the grating less than 10 cycles/deg), we could resolve both patterns and saw a central square, distinguished from the background by the orientation of the lines, standing out clearly in front of the background; it seemed no different from any fine-textured stereogram. At viewing distances between 0.5 and $2 \mathrm{~m}$, however, we had little or no impression of depth, even though we could clearly resolve and distinguish the 2 patterns and easily discriminate the central square from the background.

We confirmed our impressions of a loss of depth with high spatial-frequency stimuli by using a forced-choice test, with the results shown by the open circles in Figure 18. The subject first fixated on a pair of reference points, and then the stimuli came on for $1 \mathrm{sec}$. He was then required to say whether the central square was in front of or behind the plane of the background square. We repeated this at various distances, with the disparity, $\pm 14^{\circ}$, adjusted for viewing distance. The score fell to $75 \%$ at about 13 cycles/deg. Since stereopsis failed for spatial frequencies at which we could still easily resolve the grating, we compared our stereo threshold with thresholds for discriminating the grating and for detecting a lateral displacement of the central square. Ordinarily, one can detect stereoscopic depth with retinal disparities much smaller than the minimum displacement detectable nonstereoscopically (Westheimer and McKee, 1979). We tested our positional acuity by displacing the central square relative to the background square and viewing this single stimulus binocularly. The subject guessed whether the central square was displaced to the left or right (Fig. 18, closed circles). Here the $75 \%$ point was at about 23 cycles/deg. Finally, ability to 
right eye

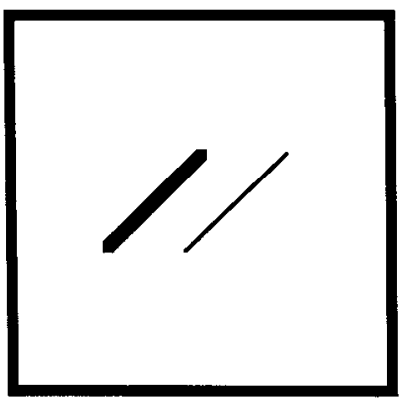

left eye

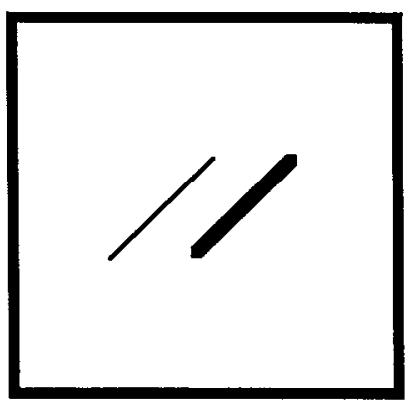

Figure 15. Test for the strength of point-to-point brightness correspondence in stereomatching. The 2 lines usually fused in favor of positional correspondence and not thin-to-thin and thick-to-thick, so subjects saw 2 lines in the plane of the frame, with some rivalry because of the luminance difference. Only occasionally, and then transiently, did the 2 thin lines fuse with each other and the 2 thick lines fuse, so that the thick line was seen in front and the thin line behind. It therefore seems that there is not a strong tendency to maximize the number of points in each retinal image whose luminosities are equal.

discriminate the grating was tested by forced-choice discrimination of the orientation of $a+45^{\circ}$ or $-45^{\circ}$ grating. For this test, our score fell to $75 \%$ at about 40 cycles/deg (Fig. 18, gray circles), Thus, our loss of stereopsis at high spatial frequencies is not attributable to a loss of positional or pattern acuity.

From these results we conclude that stereopsis, like movement perception, fails for stimuli whose spatial frequency is above 10 cycles/deg, in contrast to the form system, which can resolve stimuli above 30 cycles/deg.

\section{Loss of retinal rivalry at high spatial frequencies}

When we made the orientations of the background gratings orthogonal for the 2 eyes (Fig. $17 \mathrm{c}$ ), the stimuli were rivalrous, and the results were exactly the reverse of those for the stimuli in Figure $17 b$. At viewing distances of less than $0.5 \mathrm{~m}$ the rivalry made fusion difficult, but when the images did fuse we had no depth sensation. Because of the rivalry, the background squares did not blend, but rather appeared as a mosaic of patches of one or another orientation, which slowly shifted their boundaries and took turns fading in and out. When we stood back at an intermediate distance, between 0.5 and $2 \mathrm{~m}$, to our surprise the rivalry of the backgrounds disappeared, and we saw a uniform grid of tiny squares, like mosquito netting. The white square then stood out clearly in front. At viewing distances greater than $2 \mathrm{~m}$, the background square appeared uniformly gray and the white square appeared to lie in front of it, just as in Figure 17a. The results of forced-choice testing for the nonrivalrous and rivalrous stimuli (Fig. 17, $a$ and $c$ ) are shown in Figure 19.

Next, using the doubly rivalrous image shown in Figure $17 d$, we saw no depth at any viewing distance. $\Lambda \mathrm{t}$ close distances the images in the 2 eyes were rivalrous and could not be fused. At distances greater than $2 \mathrm{~m}$, both the central square and the background appeared to be the same uniform gray, and we saw only one large gray square. At intermediate distances we could see the gratings, but they blended into a grid without rivalry, both for the central square and the background. But even though both the center and the surround were seen as identical grids, the border between the 2, while subtle, nevertheless persisted, giving a peculiar effect of a border separating 2 identical regions, but without any difference in apparent depth.

Finally, with no central square, but just 2 large squares with orthogonal gratings, as in Figure $17 e$, the patterns were seen as rivalrous at close viewing distances, merged to give a grid at intermediate distances, and appeared a uniform gray at far distances.

The results of these experiments with stereopsis and rivalry at high spatial frequency (Fig. 17, $a-d$ ) are summarized in Table 5. From these results we conclude that binocular rivalry fails at spatial frequencies above $10 \mathrm{cycles} / \mathrm{deg}$, just as stereopsis does.

\section{Loss of binocular rivalry at equiluminance}

Given our obsession with distinguishing the different roles played by magno- and parvocellular pathways, the idea that rivalry, like stereopsis and movement, fails at high spatial frequencies suggested to us that it, too, may depend on magnocellular function. To test this idea further we looked at the effect of equiluminance on the binocular rivalry of colors. We found it was indeed much easier to fuse a pair of red and green squares so as to yield yellow if the 2 were equiluminant (Fig. 20a). At nonequiluminance, retinal rivalry was strong and the colors did not fuse, but instead showed a patchy, shimmering, unstable right eye left eye
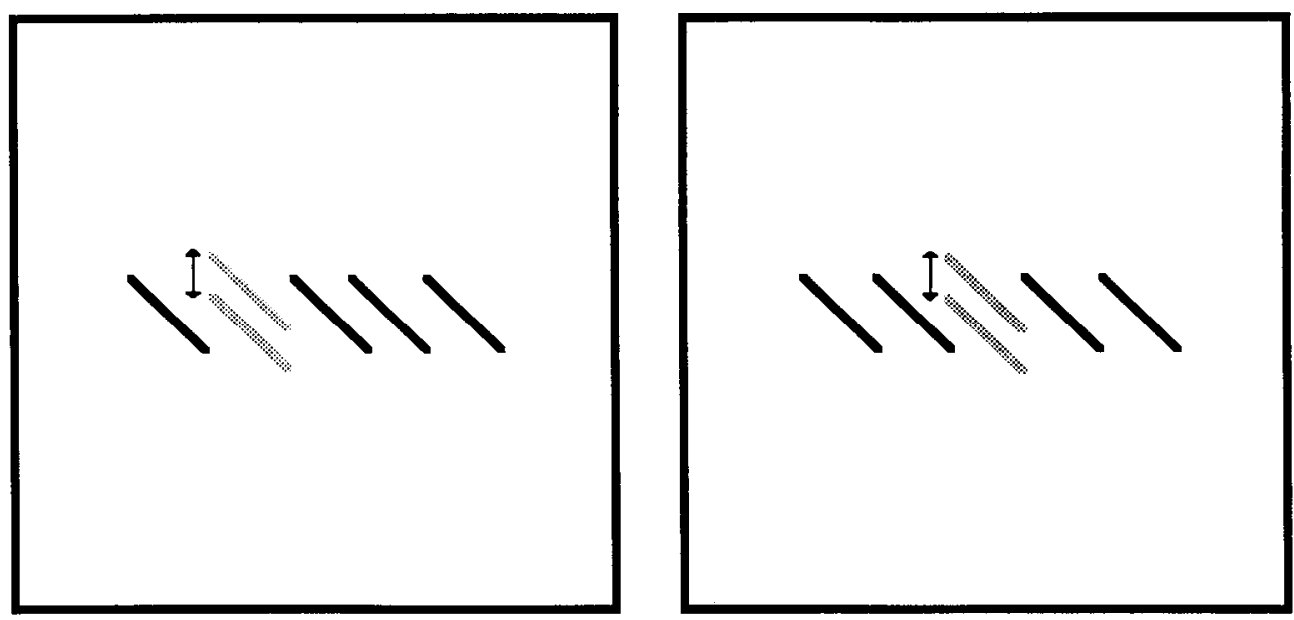

Figure 16. Influence of movement on stereomatching. In the 2 figures, one of the bars in each eye shifted up and down in synchrony. Despite the positional noncorrespondence in the 2 eyes, the moving lines dominated the pairing and a moving line was seen in front of the frame, along with 3 adjacent stationary ones. Movement thus seems to have a powerful influence on stereomatching. 
Right eye

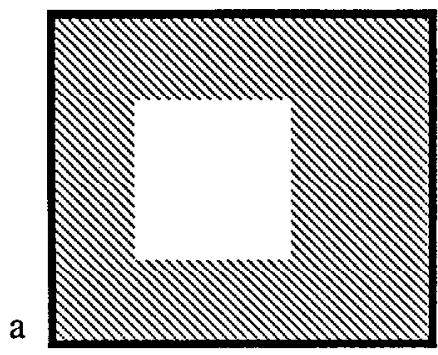

$\mathrm{b}$

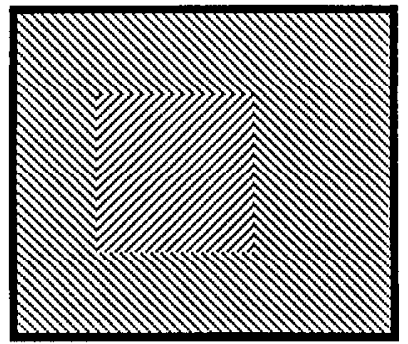

c

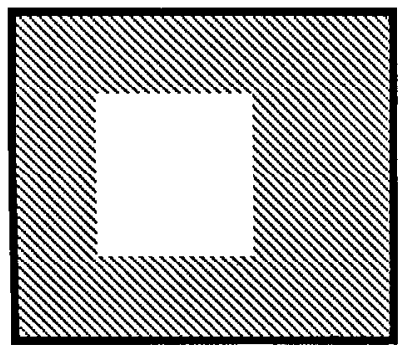

d

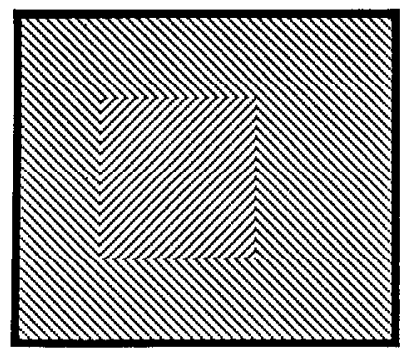

e

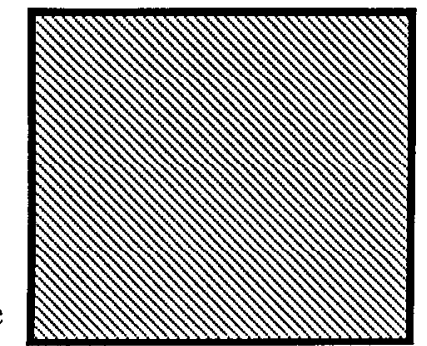

Left eye
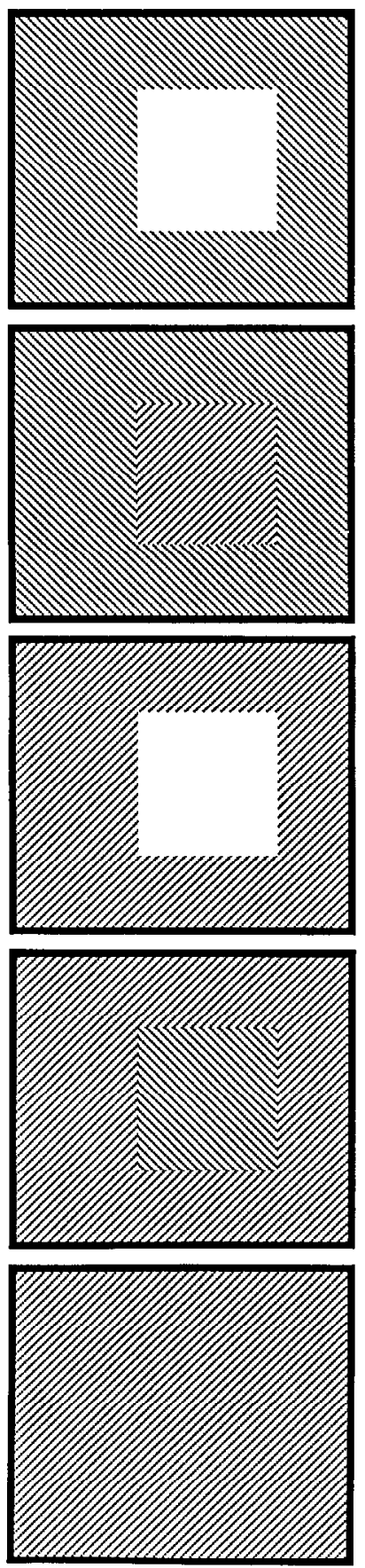

Figure 17. Five stereopairs designed to examine the influence of spatial frequency on stereopsis and on retinal rivalry. The pairs were viewed at 3 distances, near $(<0.5 \mathrm{~m})$, intermediate $(0.5-2 \mathrm{~m})$, and far $(>2 \mathrm{~m})$, at which distances the spatial frequency of the grating was less than 10 cycles/deg, $10-30 \mathrm{cycles} / \mathrm{deg}$, and over $30 \mathrm{cycles} / \mathrm{deg}$. For pair $a$, the central square appeared to lie in front of the background square at all 3 viewing distances; beyond $2 \mathrm{~m}$ the grating could not be resolved and the background appeared a uniform gray. For pair $b$, at near viewing distances the central square appeared to lie in front of the background square; between 0.5 and $2 \mathrm{~m}$ there was no sensation of depth, even though the gratings could still be resolved and the central square distinguished from the background by the difference in grating orientation; beyond $2 \mathrm{~m}$, subjects could not resolve the gratings, the central square and the background merged to a uniform gray, and we saw no depth. For pair $c$, the orthogonally oriented background gratings were rivalrous at close distances, and subjects could see no depth; at intermediate alternation from red to green. When the display was a square with the top half red and the bottom half green for one eye and the reverse for the other, as in Figure $20 b$, at nonequiluminance rivalry occurred as before, but at equiluminance the colors suddenly fused and the square looked uniformly yellow, except for a thin, faint, horizontal line across the middle, which persisted like the grin of a Cheshire cat.

The finding that color rivalry disappears most easily at equiluminance may explain differing views on binocular color fusion (see Helmholtz, 1910).

It seems, in summary, that stereopsis resembles movement perception in being color-blind, operative at low luminance contrasts, and in having lower spatial resolution than form perception. This all supports the idea that both functions depend on the magnocellular system and not on the parvo. It is not clear to us how the lower spatial resolution of the magno system can be reconciled with, or indeed if it conflicts with, the known high precision of stereo and movement hyperacuity (Westheimer, 1981) - though, as pointed out by Westheimer (1979), the type of precision exhibited in "hyperacuity" tasks probably relies on mechanisms different from those that underlie ordinary visual resolution tasks. Finally, the fact that retinal rivalry seems to share all these properties of stereopsis suggests that rivalry and stereopsis are closely related. Consistent with this idea, human developmental studies show that interocular rivalry and suppression develop at the same time as stereopsis (see Shimojo et al., 1986, for a review).

\section{Form and color}

We have already discussed the relationships between color and movement and between color and stereopsis, and have concluded from the psychophysical evidence that color-contrast information alone is relatively or totally inadequate for the mediation of movement or stereopsis. We now turn to the relationship between color and form perception, for several reasons a more difficult topic. A clean separation of form and color would hardly be expected, since form, in the sense of contours, is necessary in order for color to exist at all, and since one can perceive form from pure color-contrast information. Anatomically and physiologically, the evidence favoring separate pathways for color and form is weaker than that for color versus movement or for color versus stereopsis, but there is evidence for some degree of separation. The magnocellular system probably cannot make use of color-contrast information; the parvocellular system probably can use it to help in defining contours (using the cells with occult color coding in the interblob regions of area 17), but the blob system is likely responsible for the actual determination of color as such, i.e., hue and brightness.

\footnotetext{
$\leftarrow$

distances, the 2 background gratings fused, without rivalry, into a crosshatching, and the square stood out clearly in depth; at far distances, the background gratings appeared uniform gray, and the white square was seen in front. Pair $d$ was rivalrous for the center and background at close range, and there was no sensation of depth. At intermediate distances, subjects saw cross-hatching over the entire image; there was no sensation of depth, but the center square was nevertheless still distinguishable from the background, even though both appeared to be the same crosshatching. Farther away still, the entire square was uniform gray. Pair $e$ gave, at the 3 viewing distances, rivalry, uniform cross-hatching, and uniform gray. We conclude that at high spatial frequencies stereopsis and retinal rivalry fail before form discrimination. (Forced-choice tests were made for pair $b$; see Fig. 18. For pairs $a$ and $c$, see Fig. 19.)
} 


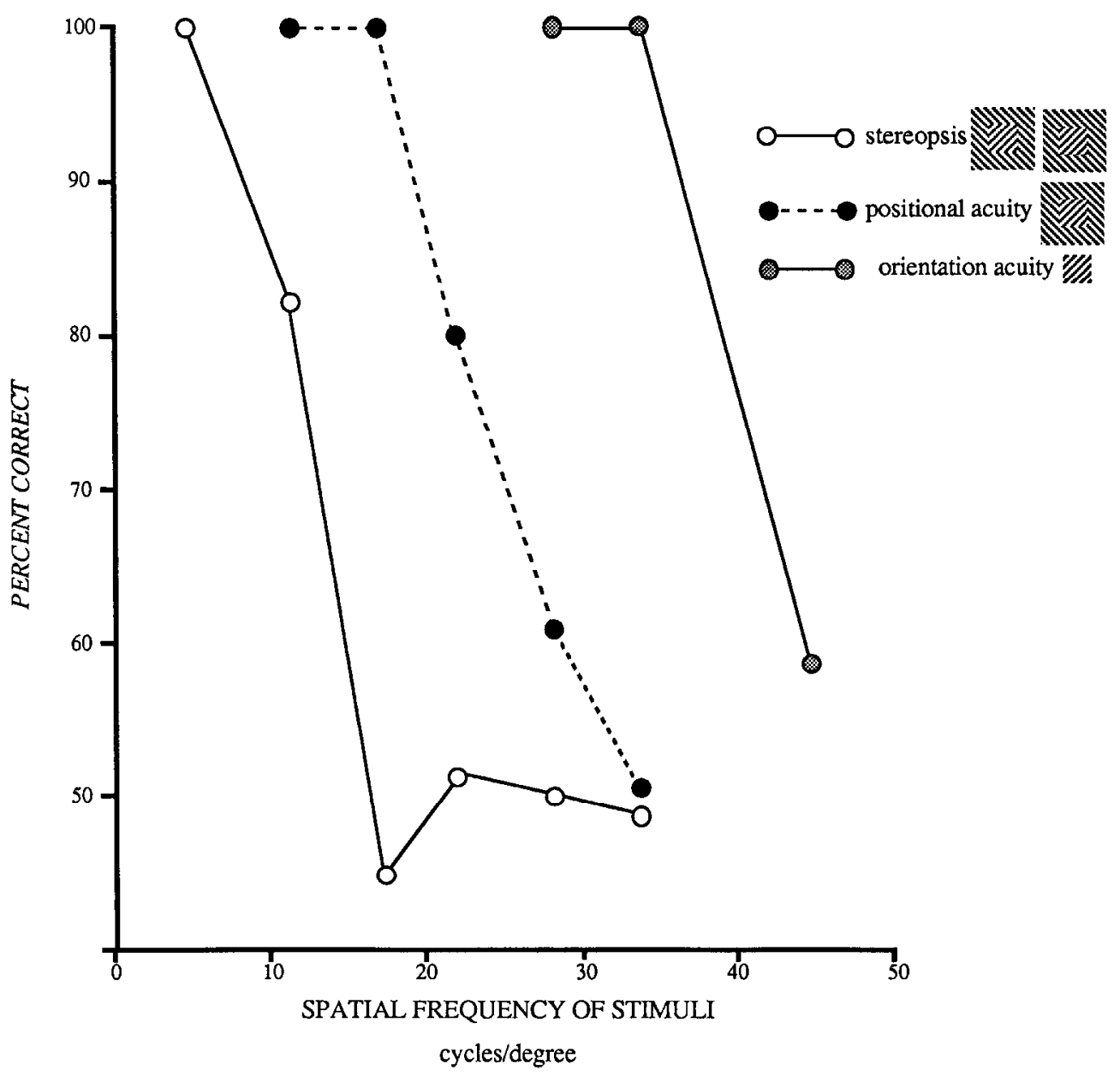

Figure 18. Comparison of stereopsis, positional acuity, and orientation acuity as a function of spatial frequency; forced-choice testing. Using the stereopair in Figure $17 b$, and one with the opposite relative displacements, we measured subjects' ability, as a function of spatial frequency, to discriminate a square in front from one behind; this fell below criterion (75\%) at about 13 cycles/deg. The ability to distinguish the left and right members of these stereopairs, differing only in the left-right position of the central square, is shown by the filled circles. Criterion was at 23 cycles/deg. As a measure of form acuity, we tested the ability to distinguish the 2 line orientations (gray circles). This fell to $75 \%$ at 40 cycles/deg. Thus the stereopsis could not have failed at 13 cycles/deg because of an inability to detect the shift in position or the orientation difference.
If it is permissible to equate form with orientation selectivity, then the magno and parvo systems would both carry form information, but the blob system would not. An obvious difficulty in discussing form perception is that it is probably not a single, or simple, process; the possibility that it may be handled by both the parvo and the magno systems immediately makes one wonder if these 2 systems do not handle fundamentally different aspects of form perception. The motivation behind the experiments described below was the notion that it might be possible to distinguish different aspects of form perception by using the differences between the magno and parvo systems.

\section{Form perception at low luminance contrast}

Given the high contrast sensitivity of the magno system compared with that of the parvo, an obvious question to ask is,
What kinds of form perception are possible at low luminance contrasts? We therefore first tested our ability to distinguish simple forms, a square and a circle, using stimuli of different sizes at different luminance contrasts. As shown in Figure 21, we could make this distinction at very low levels of luminance contrast. Thus there was no difference in threshold luminance contrast between this simple kind of form perception and stereopsis or movement perception. But with increasing contrast, the rangc over which form perception showed improvement extended to higher contrasts than that for stereopsis or movement. Comparing Figures $6 b, 12 b$, and $21 b$, the dynamic range of luminance contrasts for stereopsis and movement is lower than that for form, and in fact is similar to the range described for magnocellular units, which saturate at $20-30 \%$. For form perception, on the other hand, the high sensitivity at low luminance

Table 5. Loss of stereopsis and ocular rivalry with high spatial-frequency stimuli (Fig. 17)

\begin{tabular}{|c|c|c|c|c|c|}
\hline \multirow{2}{*}{\multicolumn{3}{|c|}{$\begin{array}{l}\text { Spatial frequency } \\
\text { information }\end{array}$}} & \multicolumn{3}{|l|}{ Percept } \\
\hline & & & \multicolumn{3}{|c|}{$\begin{array}{l}\text { Grating spatial frequency } \\
\text { (cycles/deg) }\end{array}$} \\
\hline & High & Low & $<10$ & $10-30$ & $>30$ \\
\hline$a$ & Yes & Yes & Square in depth & Square in depth & Square in depth \\
\hline$b$ & Yes & No & Square in depth & Square-no depth & No square \\
\hline$c$ & Rivalrous & Yes & Rivalry-no depth & Square in depth & Square in depth \\
\hline$d$ & Rivalrous & No & Rivalry-no depth & Square-no depth & No square \\
\hline
\end{tabular}




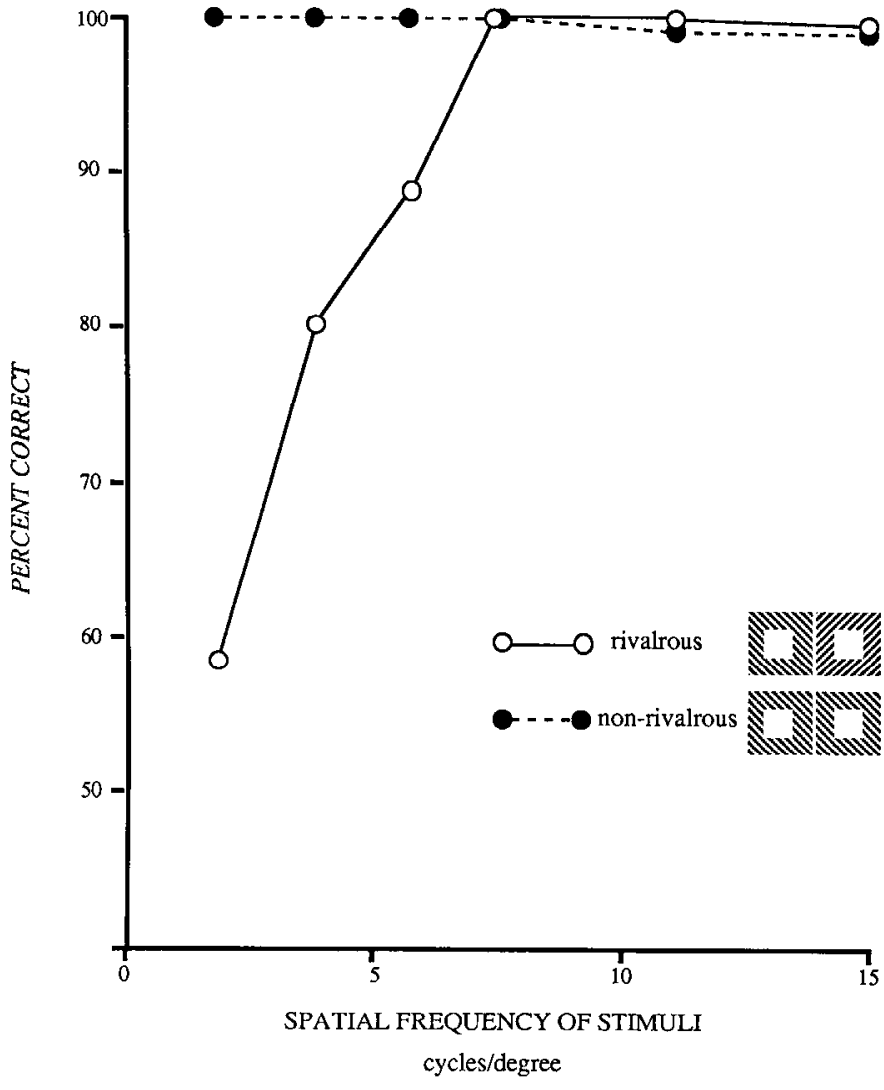

Figure 19. Loss of rivalry at high spatial frequencies; forced-choice testing. We measured subjects' ability to discriminate stereoscopically a square in front from a square behind, for Figure 17, pair $a$ (backgrounds not rivalrous, closed circles) and pair $c$ (backgrounds rivalrous, open circles), as a function of spatial frequency. Rivalry interfered with stereopsis at low spatial frequencies, but rivalry was not present, and did not interfere with stereopsis, for spatial frequencies above about 10 cycles/ deg.

contrasts is characteristic of the magno system, but the lack of saturation up to contrasts of $50 \%$ correlates better with the properties of the parvocellular system (Kaplan and Shapley, 1982; Derrington and Lennie, 1984).

We therefore conclude that both the magno and the parvo systems can contribute to form perception. A study by Tansley et al. (1983) suggests that there are indeed 2 processes contributing to form perception. They compared minimal border distinctness and pattern-evoked cortical potentials as functions of color contrast and luminance contrast, and found that the colorcontrast-sensitive function had only one component, whereas the luminance-contrast-sensitive function was biphasic, one component having a higher contrast sensitivity. We suggest that this achromatic, high-sensitivity component is carried by the magno system.

\section{Achromatopsia}

The most powerful evidence that color and form perception are carried by separate pathways comes from clinical neurology. Cortical achromatopsia is a well-recognized if rare syndrome of selective loss of color perception due to damage to a visual region anterior to area 18 . It is not known whether this region is homologous to macaque area V4. One patient described by $\mathrm{Da}$ masio et al. (1980) attracted our attention not only because of her description of colors being "drained out" and "not true," but because of her description of white objects' looking dirty. "She also had every drapery in the house cleaned because they looked dirty to her. They continucd to look so on their return from the cleaners. She complained that the snow was gray and dirty also but nothing much could be done about it" (Damasio et al., 1980). This interested us because we had wondered, given the presence of many broadband cells in the blob/thin-stripe system, whether patients with selective damage to a prestriate cortical color area would show defects in perception of blacks, whites, and grays. We examined this patient, but by then she had virtually recovered her color capabilities. We also examined a second patient with unilateral cortical acquired achromatopsia. He had a total loss of color discrimination in the visual field contralateral to his stroke, with no obvious loss of form vision. When we showed him, in his color-blind visual field, 2 pieces of colored paper, for example blue and orange, he said they both looked gray, but he could see that they were different. When we varied continuously the luminance contrast between the 2 pieces of paper by varying the strength of illumination from 2 light sources, one red and the other blue, he still saw the border between the 2 pieces of paper, even when the illumination was such that he judged them to be of the same brightness. This observation, which we could not make with any rigor, would be interesting if correct, since an ability to detect equiluminant color borders in the absence of the ability to discriminate colors as such is what would bc expected if the form system got input from color-coded cells, but pooled the information in such a way that the sign of the color contrast was lost. Under ordinary light, the patient generally saw 2 differently colored papers as being different shades of gray. He was able to distinguish and order correctly pieces of paper of different shades of gray pasted at random along a wall, using only his color-blind visual field, whether the papers were illuminated head-on or obliquely.

Thus our scanty observations on these 2 patients give us conflicting conclusions as to whether the color system in humans also carries information about black, white, and grays. Obviously we would like to test other patients with cortical achromatopsia.

\section{Bleeding and contrast}

Under this category we include 2 hemorrhagic phenomena: Bezold bleeding and neon bleeding. In both, a colored region imparts its color to an adjacent region of different hue.

When 2 regions of different colors are juxtaposed, especially if one region is surrounded by another, the perceived color is usually different from what is seen against a noncolored background: the center region tends to look more like the opposite of the surround (towards the complement of the surrounding hue, and lighter when surrounded by a dark background, darker on a light background). This phenomenon is called "simultaneous contrast." In certain geometric arrangements, however, the interaction between 2 adjacent regions is just the opposite. When one of the colors forms a fine, but still resolvable, pattern subdividing the other color, the color of the fine pattern tends to bleed into the other color. This has been called Bezold bleeding (von Bezold, 1876), but was described even earlier by Chevreul (1839) for tapestry threads. If the fine pattern is blue, a red background becomes more purple; if it is yellow, the red background becomes orange; fine dark patterns make a background appear darker; light patterns lighten the background. Both bleeding and contrast are illustrated in Figure 22. 


\section{PERCEPT}

\section{STTMULUS}

a.

right eye
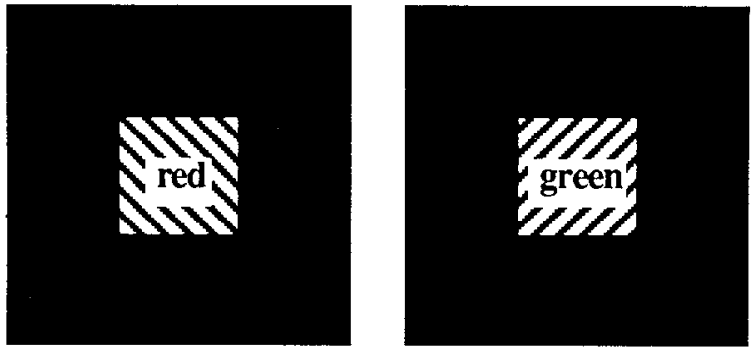

b.

right eye

left eye

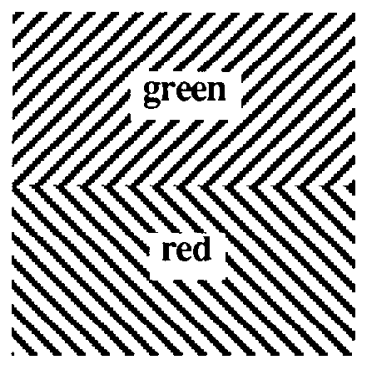

left eye at non-equiluminance

at equiluminance
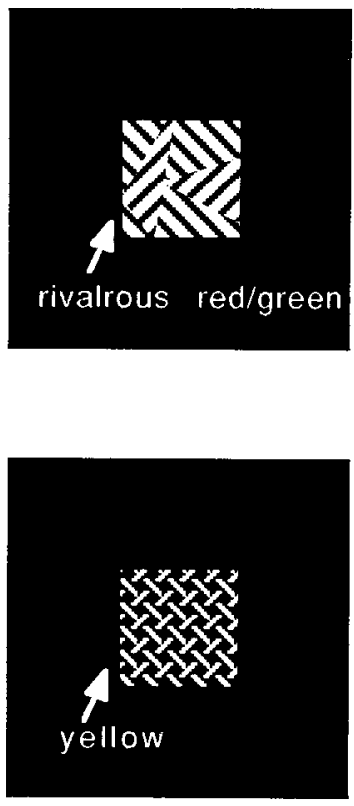

at non-equiluminance

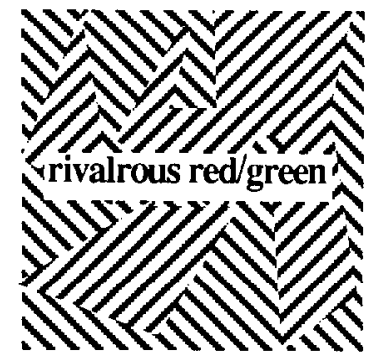

at equiluminance

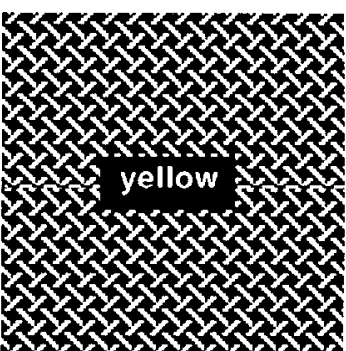

Figure 20. Binocular fusion of colors. $a$, If the right eye views a red patch and the left eye a green patch, the result is usually color rivalry, in which one sees a shimmering, irregular, and constantly changing patchwork of red and green. When the red or the green intensity is varied over a narrow range of red-to-green ratios, the 2 fuse to produce a homogeneous yellow (binocular yellow; Helmholtz, 1910 ). In $b$, each square is divided in half, so that a red region in one eye corresponds to a green region in the other. Again, at nonequiluminance, one sees an irregular, unstable mosaic of red and green, but at equiluminance the 2 images fuse to give yellow, with a faint persistent horizontal line.

It is tempting to interpret the bleeding in terms of the spatial resolution of the color system: The field centers of color-coded blob cells are at least twice as large as the field centers of cells in the interblob system (Livingstone and Hubel, 1984a). We suggest that bleeding occurs when the fine pattern can be resolved by the form system but not by the color system. From the physiology of center/surround cells, we can deduce that it is the cells whose receptive-field centers are to either side of the 


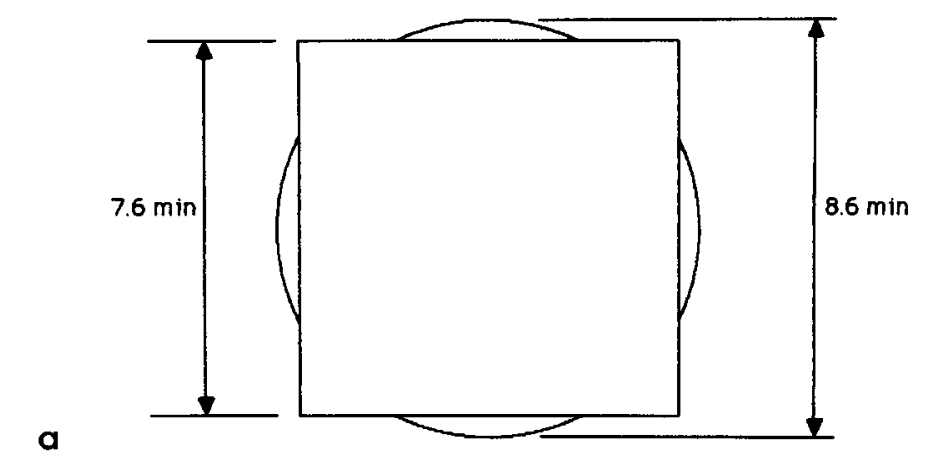

Figure 21. Contrast sensitivity of form discrimination; forced-choice testing. $a$, Either the square or the circle was presented, at the point of fixation, for $1 \mathrm{sec}$, and the subject had to guess which. The areas of the two figures were the same. Three sizes of each were used. (The sizes indicated are the length of a side of the square.) $b$, The graph plots percentage correct against contrast. The results indicate that form discrimination is possible at contrast levels of a few percentages, but that it continues to improve with increasing contrast above $40 \%$.

\section{FORM}

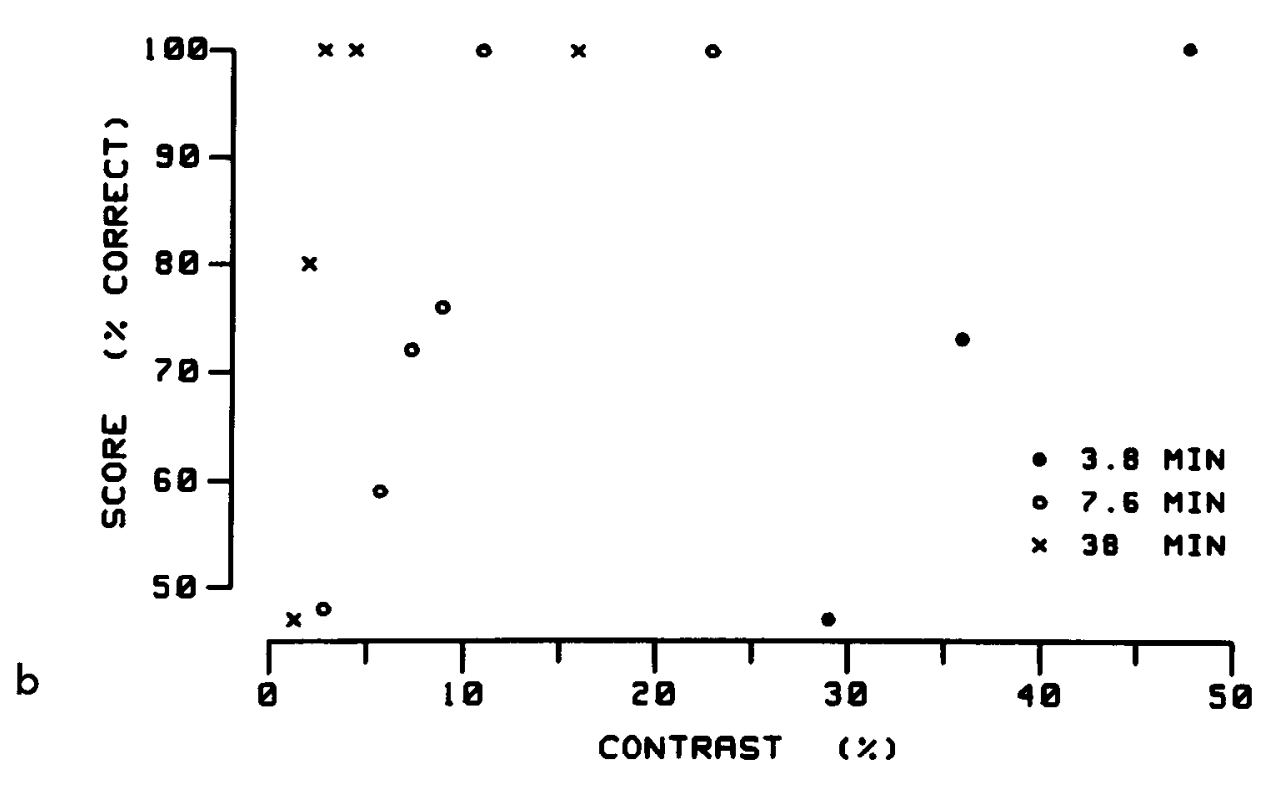

borders separating spectrally different regions that signal color, not the cells whose receptive fields are totally within the regions. Bleeding occurs when patterns are fine, that is, when the borders bounding colored regions are close together, perhaps close enough that opposite borders of the fine-colored regions can both fall within the receptive-field center of a single double-opponent cell.

Bleeding, like simultaneous contrast, occurs not only between colors but also between shades of gray, black, and white (Fig. 22 , bottom), consistent with the idea that the lower-resolution system (the blob system) is concerned with black and white and shades of gray, in addition to colors (Livingstone and Hubel, 1984a).

In a typical magazine illustration, a pink is produced by printing red dots, too small to be resolved, on the white page. Suppose one were to view the pink region under steadily increasing magnification, until the dots became resolvable. At that moment would the dots be a rich red, and the page suddenly chalk white? The differences in acuity of the color and form systems suggest that they would not-that only well after the dots became resolvable would their color appear confined to the dots.

A variant of this bleeding phenomenon is known as "neon bleeding" (van Tuijl, 1975; van Tuijl and de Weert, 1979), illustrated in Figure 23. Neon bleeding is the spread of color out of fine lines within an illusory border (illusory borders are discussed below). The figure defined by the illusory border ap- pears brighter than the background because of the sign of the contrast at the transitions that generate the illusory border. It is the combination of the color and the subjective brightness that makes the illusory color seem to glow; thus the term "neon." In the examples shown, the white space between the red or blue lines seems to be pink or light blue. The illusory color extends only to the illusory border created by the boundary between the colored and the black lines. The spreading color is stopped just as well by real borders as by illusory ones, but the phenomenon seems to be more noticeable with illusory borders, perhaps because the region into which the bleeding occurs and the region beyond are immediately juxtaposed, without any real border intervening.

\section{Contribution of edge information to color perception}

The idea that contours are essential for color perception comes largely from the work on stabilized images by Krauskopf (1961) and Yarbus (1967). They found that when part of an image is stabilized on the retina, that region first becomes an empty gray, and then, within a second, fades to the same color as its unstabilized surround (which remains visible). If the surround is uniform in color, and so large that its borders are outside the subject's visual field, both the surround and the stabilized region lose all form and color. The fact that one sees the object's color (including blacks, whites, and grays) only if its edges are not stabilized implies that the borders are the important thing for 

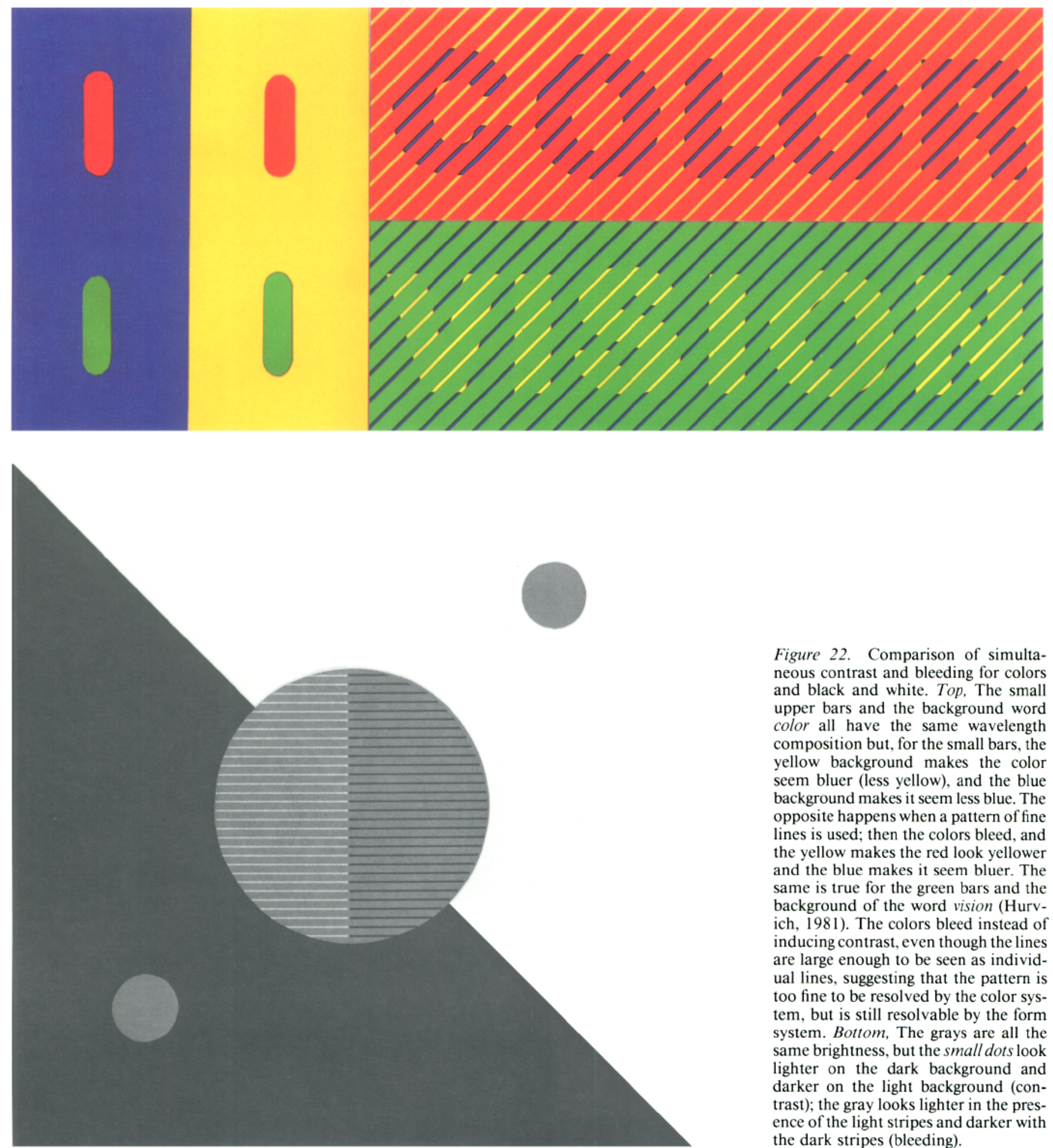

Figure 22. Comparison of simultaneous contrast and bleeding for colors and black and white. Top. The small upper bars and the background word color all have the same wavelength composition but, for the small bars, the yellow background makes the color seem bluer (less yellow), and the blue background makes it seem less blue. The opposite happens when a pattern of fine lines is used; then the colors bleed, and the yellow makes the red look yellower and the blue makes it seem bluer. The same is true for the green bars and the background of the word vision (Hurvich, 1981). The colors bleed instead of inducing contrast, even though the lines are large enough to be seen as individual lines, suggesting that the pattern is too fine to be resolved by the color system, but is still resolvable by the form system. Bottom, The grays are all the same brightness, but the small dots look lighter on the dark background and darker on the light background (contrast); the gray looks lighter in the presence of the light stripes and darker with the dark stripes (bleeding).

establishing the color. From his experiments, Yarbus concluded that "the apparent color of an empty field ... is always equal to the color of its borders." Neon bleeding is another illustration of the containment of colors by borders, in this case illusory borders.

Although some aspects of perception, such as movement perception or stereopsis, are completely lost or greatly diminished when borders consist of color contrast without luminance con- trast, other aspects of perception, such as shape discrimination, are only slightly degraded. This distinction between lost and retained abilities can be seen in single images: in the experiments with red/green Julesz stereograms, for example, the dots and their colors were still clearly resolved even when the sensation of depth disappeared. Gregory (1977) found that he lost all sensation of depth with an equiluminant random-dot stereogram made up of dots that were 12 times larger than the min- 


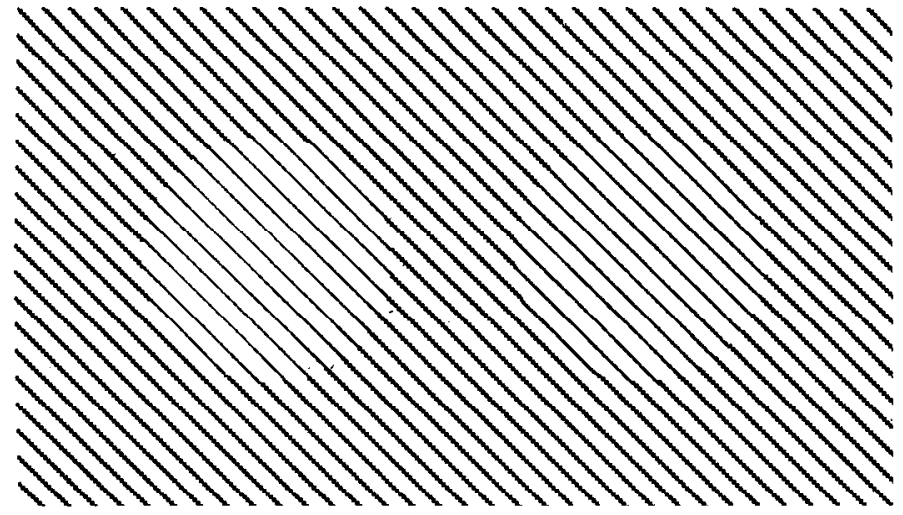

Figure 23. Neon bleeding.

imum resolvable dot at equiluminance. Similarly, at equiluminance the moving red/green sine-wave gratings of Cavanagh et al. (1984) are still seen as parallel red and green stripes; only their motion seems reduced or absent. Finally, when apparent movement was eliminated at equiluminance, as described above, the spots were plainly visible as spots, however much they danced about.

Orientation-dependent color aftcreffects, such as the McCollough effect (1965), seem to indicate that, at some stage in visual processing, orientation and color selectivity must converge; but these effects take at least several minutes to be generated and last for up to several days, suggesting that the convergence may not occur at an early stage. Indeed, the McCollough effect does not occur if the inducing grating is equiluminant color/gray stripes (Harris and Barkow, 1969; Stromeyer and Dawson, 1978), suggesting that the form information involved in this effect is carried by an achromatic system prior to any convergence with the color information.

\section{Color-contrast versus luminance-contrast acuity}

That form perception takes on a very peculiar character at equiluminance has been mentioned in several descriptions. Liebmann (1926) characterized the perception of figures at equiluminance as “. . . quallig, kolloidal, die Figur hebt sich nicht recht aus dem Grunde heraus, sie ist nicht nur etwa mit unscharfen Konturen da, sondern als ganze ist sie 'verschwommen'." (“... jelly-like, colloidal, the figure does not stand normally out of the background, it not only has blurred contours, but as a whole is hazy."). Gregory (1977) described such figures as "jazzy" and "unstable." At equiluminance, borders become so "indistinct" that the "minimally distinct border" has been used as a technique to define equiluminance (Tansley and Boynton, 1978). This psychophysical evidence suggests that visual acuity for equiluminant color-contrast borders should be lower than that for luminance-contrast borders, but this prediction seems contrary to the physiological findings that cells in the color-sensitive parvo system have smaller receptive fields than cells in the colorblind magno system.

Studies in the past that have addressed the question of luminance-contrast versus color-contrast acuity have not found significant reductions in acuity for equiluminant stimuli, and we at first thought that the failure to find a reduction in acuity might be due to problems in achieving equiluminance. Cavonius and Schumacher (1966) found no difference between color-con- trast acuity and luminance-contrast acuity, provided the wavelengths used for the color borders were far enough apart. Equiluminance in their experiments was determined by progressive stepwise brightness-matching across the spectrum, rather than by looking for a level at which acuity was minimal. A subsequent study by Granger and Heurtley (1973) found only a 2-fold falloff in acuity levels at equiluminance, but they used flicker photometry to define equiluminance. In recent studies by De Valois and Switkes (1983) and Mullen (1985), red/green intensitics were continuously varied while determining threshold amplitude of sine-wave gratings at various spatial frequencies, and the results were compared with those obtained with luminousintensity gratings. These studies indicate a reduction in foveal acuity by a factor of only 2-3 at equiluminance.

A reduction of only 2-3-fold in acuity did not seem to us enough to account either for the instability and poor image quality at equiluminance or for the breakdown of stereopsis or various illusions at equiluminance, to be described below. We found, for example, that making these illusions several times larger did not prevent the breakdown. Many of the phenomena lost at equiluminance seem to involve relating or linking perceptual features over fairly wide visual angles, and at first we wondered whether this might reflect a more severe deterioration at equiluminance and of acuity in the peripheral visual field. We therefore measured acuity at equiluminance at different eccentricities, by 2 criteria. We determined by forced-choice the acuity for (1) a Landolt-C gap and for (2) distinguishing a circle and square of the same area. For each test, at each eccentricity, we compared acuity for black-white stimuli to acuity for colorcontrast stimuli over a broad range of continuously variable relative intensities that included equiluminance, as determined by both flicker fusion and the range, with about $20 \%$ less green, in which most other phenomena were minimized. For each determination we gradually changed the relative red/green ratio to find the setting at which acuity was lowest. Each presentation was brief $(100 \mathrm{msec})$. At all eccentricites the acuity at equiluminance fell by at most a factor of 2 . These results suggest that color-contrast acuity does not fall off with eccentricity any more than luminance-contrast acuity does, and (in agreement with Mullen) that the difference in acuity between color-contrast and luminance stimuli is clear but not large. We therefore conclude that some aspects of form vision, especially aspects involving the coherence of the visual fields or the relating of objects over wide areas, may be color-blind in the same sense that stereopsis and movement are, but that other aspects, especially those having to do with the analysis of shape or contours, are not. If the parvo/interblob system is responsible for the analysis of form, perhaps a 2-fold loss in form acuity at equiluminance is related to the finding that about half the cells in the interblobs become unresponsive at equiluminance (Crouras and Krïger, 1979).

A recent study by Morgan and Aiba (1985), which reports that vernier acuity was reduced severalfold at equiluminance, seems to be in disagreement with the idea that the parvo system is not greatly affected by equiluminance and is responsible for high-resolution form perception. But we do not think highacuity form perception necessarily includes the types of abilities that are called hyperacuity, and suspect that those functions may well be carried out by the magno system. One reason for thinking this is that subjects can exhibit resolution of a few arc seconds for both motion detection and stereo acuity tests, (Westheimer, 1981), both functions that we think may be carried out by the magno system. We would not be surprised if posi- 
tional or vernier-offset acuity were also attributable to the magno system. The observation that positional hyperacuity involves sampling over a much larger distance than the distinguishable offset (Levi and Klein, 1986) would be consistent with the spatial integrative abilities of the magno system, which we will describe below.

\section{Fading of images at equiluminance}

The modest measured lowering of acuity at equiluminance seemed to us not to fit with the descriptions of equiluminant figures in the literature - for example, the accounts of Liebmann (1926) and Gregory (1977)-or with our own impressions. Krauskopf (1967), Liebmann (1926), and Gregory (1977) had all observed a tendency for equiluminant images to fade. We had also found that fine color-contrast patterns tended to disappear, often completely, at equiluminance. We were at first mystified by the transience of the phenomenon, the seemingly irregular and unpredictable way in which the text or pattern would disappear and reappear. We finally found that fixing our gaze on any point caused the entire pattern to vanish after about a second, and that voluntarily shifting our gaze to a new point caused the display to reappear immediately. It indeed seems, as was previously described by Krauskopf (1967), that equiluminant images tend to fade with voluntary gazc fixation, just as luminance-contrast images fade when the image is artificially stabilized (Ditchburn and Ginshørg, 1952; Riggs et al., 1953).

We tested the propensity of spots to fade at equiluminance, at various eccentricites and for various spot diameters. To our surprise, we found that even a large spot or collection of spotssuch as those shown in Figure 24-would disappear under voluntary fixation. The edges of the spot or spots first became blurred; this was followed by a complete merging with the background. For the array of Figure $24 a$, what was left was not yellow, as might have been expected from mixing red and green, but a grayish wash that gradually merged with the green above. The green was itself somewhat desaturated, presumably because of the many seconds of fixation. In all cases where fading occurred, the spot or spots reappeared at once if we changed the red-togreen ratio. Because, except for targets so large that variations in cquiluminance with eccentricity may be a factor, the tendency to fade did not seem to correlate with image size, we conclude that it is the edges, or contours, rather than the interiors of the spots, that disappear with fixation.

Since observer D.H.H. was (and is) totally presbyopic, we assume that failure of accommodation (Wolfe and Owens, 1981) probably plays no primary role in these effects. Rather, we suspect that accommodation fails at equiluminance simply because the images one is trying to focus on fade.

In order to attribute the fading of equiluminant images to the same cause as the fading of artificially stabilized images, one need only assume that, for equiluminant images, the resolution of the system is low enough that the eye movements persisting during steady fixation are no longer sufficient to renew the image. During maintained voluntary fixation there are 3 kinds of eye movements: microsaccades or flicks, slow drifts, and tremors. Of these, it is thought to be either the flicks (microsaccades) (Ditchburn et al., 1959) or the slow drifts in eye position (see review by Alpern, 1972) that serve to refresh the image and prevent fading. Since flicks can apparently be voluntarily suppressed for many seconds during steady fixation, the slow drifts alone are thought to be sufficient to maintain the image (Stein-
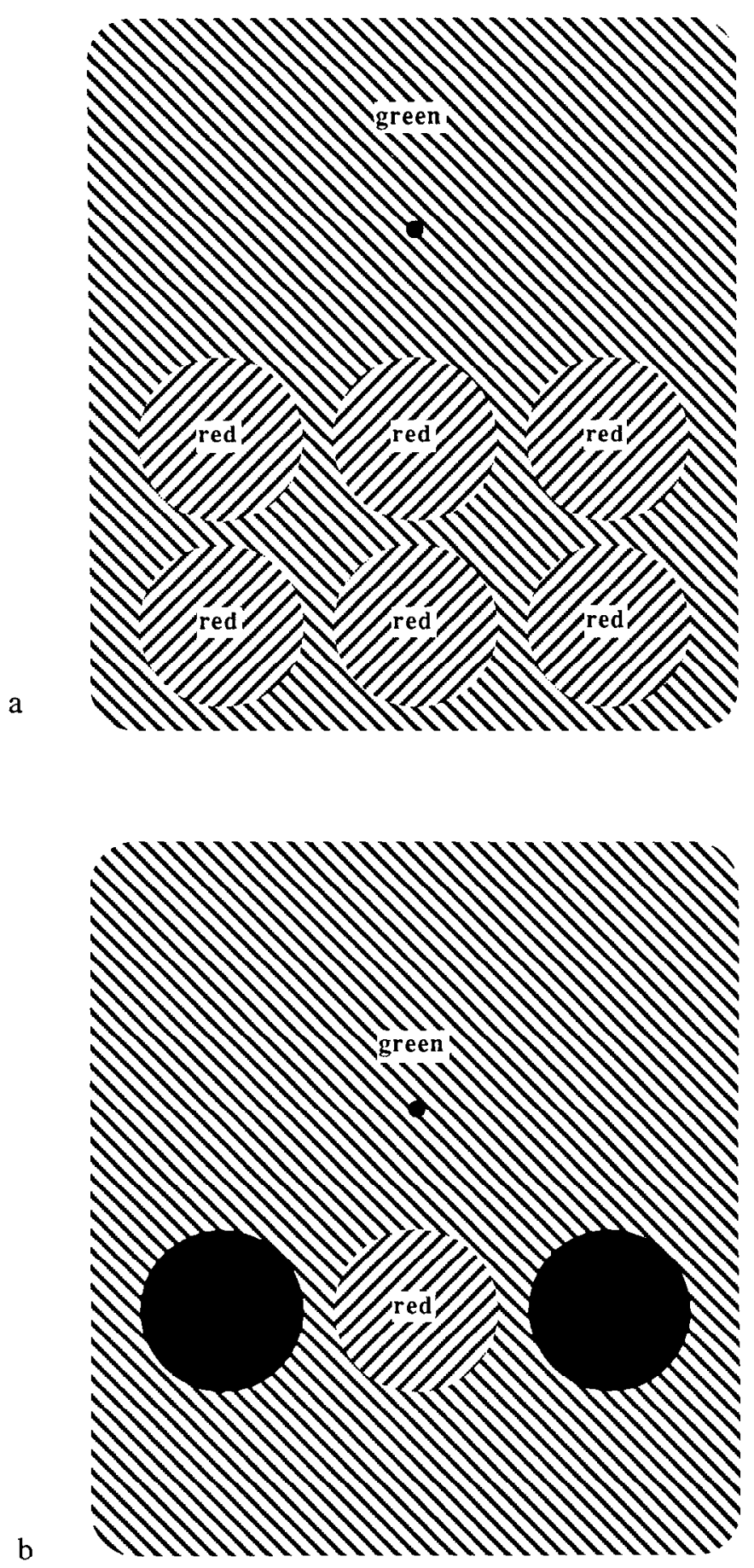

Figure 24. Two displays illustrating the disappearance of equiluminant borders with voluntary fixation. The large $\left(3^{\circ}\right)$ circles were red on a green background, and the green intensity could be continuously adjusted. $a$, When the subject fixated on the small black circle and adjusted the green intensity, a range could easily be found in which all the red circles vanished. $b$. The 2 outer $3^{\circ}$ circles were black. When the red and the green were equiluminant, the center red circle vanished with fixation, but the black circles, which were not equiluminant, did not.

man et al., 1967). Slow drifts were found by Ditchburn and Foley-Fisher (1967) to have an amplitude of 0.8-6 arc min, with a median value, in 16 subjects, of about $2.5 \mathrm{~min}$. With a retinally stabilized image, one can introduce a sudden movement of the image and restore its visibility; the size of the displacement 


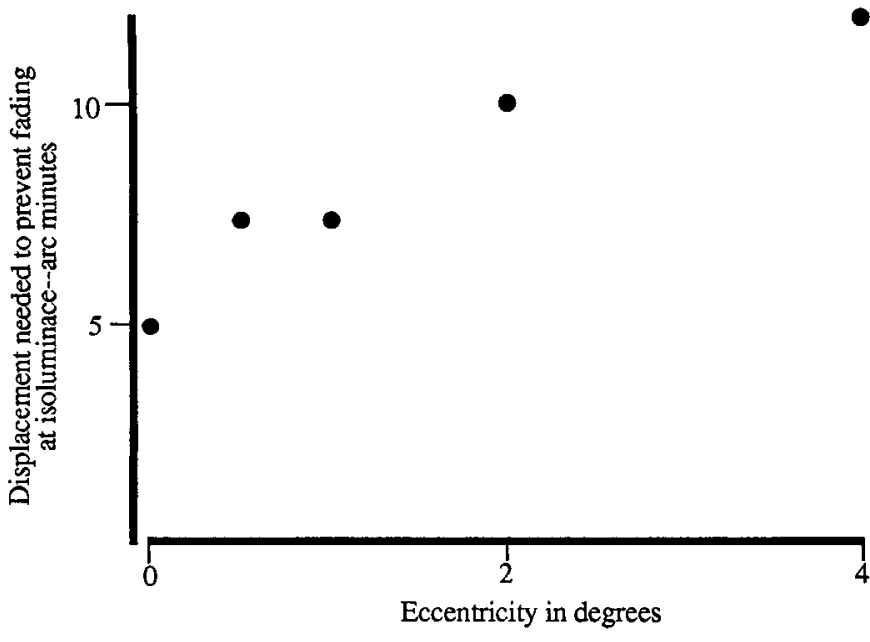

Figure 25. Size of displacement needed to prevent fading (with voluntary fixation) of an equiluminant spot as a function of eccentricity. The display consisted of a $1 / 2^{\circ}$ spot, red on a green background, at various distances from a small fixation point. For cach cccentricity, the intensity of the green background was adjusted so that the spot disappeared. We then made the spot move back and forth in position horizontally over a variable distance and asked whether the spot still disappeared with fixation. The graph shows the relationship between eccentricity and the smallest displacement that resulted in the spot's persistence. Even foveal spots vanished if the displacement was less than 5 arc min.

necessary to restore visibility in an artificially stabilized image is 2 arc min, about the same size as the slow drifts (Ditchburn et al., 1959). Will an equiluminant image that has faded under voluntary steady fixation reappear if it is displaced through an excursion of this size?

We approached this question by measuring the displacement necessary to overcome fading of an equiluminant spot. We used a $1 / 2^{\circ}$ spot whose position alternated horizontally back and forth at 1 cycle/sec. The spot showed apparent movement as long as it was not equiluminant with the background, but at equiluminance it merely danced on and off in the 2 positions, as we have already described. At eccentricities ranging from $0^{\circ}$ to $4^{\circ}$, we asked whether fading occurred for various movement excursions. For a given eccentricity, complete fading occurred and persisted for many seconds despite displacements up to some fairly sharply defined distance, but for larger displacements, the spot reappeared immediately with each displacement. A plot of the threshold displacement needed to prevent fading at various eccentricities is shown in Figure 25. Evidently movements of 5-10 arc min are necessary to prevent fading at equiluminance. Thus eye movements during voluntary maintained fixation would be too small, by a factor of 2-4, according to Ditchburn and Foley-Fisher's data (1967), to keep an equiluminant image refreshed.

If this explanation is correct, then we would expect that even within the same scene, an equiluminant image could fade when a nonequiluminant image would not. When we looked at the display shown in Figure $24 b$, we found that this was indeed so. When we made the red spot equiluminant with the green background, and fixated on the small black dot, the central spot vanished, but the flanking black spots did not.

Our first notion was that the fading at equiluminance might occur because activity in the magnocellular system is needed to prevent fading, since it seems to be responsible for many of the other phenomena that fail at equiluminance. A demonstration by Cornsweet (1966) that low luminance-contrast images fade with voluntary fixation suggested, however, that this might not be the explanation. We therefore asked what levels of luminance contrast were needed to prevent fading. Contrast ratios of 10$20 \%$ should be optimal for the magnocellular system, but much less effective in stimulating the parvocellular system. We examined circular gray spots of different sizes against a gray background at various contrasts. Fixation was $2^{\circ}$ from the edge of each spot. To our surprise, we found that at $20 \%$ contrast we had no difficulty in obtaining fading, even of the largest figures. Figure 26 shows the largest diameters of circles that disappeared completely with voluntary fixation as a function of luminance contrast. As contrast between spot and background increased above $20 \%$, the size of the largest nonfading spots fell, with a clear improvement even beyond a 5 -fold difference in contrast. The low contrast sensitivity and lack of saturation at high contrasts seen in this experiment suggest that it is the parvo and not the magno system that prevents fading during voluntary fixation. Our observation that the eye movements required to prevent fading at equiluminance are 2-4 times greater than the movements required at high luminance contrast could be attributed to the 2-4-fold reduction of acuity at equiluminance, which is consistent with the observations of Gouras and Krüger that many of the upper-layer, presumably interblob, cortical cells show a response minimum at equiluminance and roughly half of them become unresponsive. The color-coded blob cells should also remain active at equiluminance, and so could be responsible for the revival of images with larger movements.

We suggest, in summary, that eye movements during voluntary fixation are so small that at nonequiluminance it is only the visual subsystem with the highest resolution, presumably the parvo-interblob system, that does not adapt, and that therefore prevents images from fading during fixation. It seems that at equiluminance, the resolution of this system is reduced enough so that the same eye movements are no longer large enough to prevent it from adapting, so images fade. We suspect that this tendency for equiluminant images to fade during steady fixation can go a long way in explaining why they look so strange. An early observation of Krauskopf (1957) on the temporal requirements for movements preventing fading of a stabilized image is consistent with the idea that it is only the parvo system that does not fade. He found that in an artificially stabilized image, he could restore visibility of an object by moving it back and forth; low-frequency vibrations, 1,2 , or 5 cycles/sec, were effective in restoring the visibility of a stabilized image, but higherfrequency vibrations, 10,20 , or 30 cycles/sec, caused the image to fade even more easily than stabilization alone. As discussed below, this relatively low temporal-frequency cutoff would be consistent with the slow temporal properties of the parvo system. These results imply that during voluntary fixation, the magno system adapts to a visual image within a few seconds.

\section{Loss of classical illusions at equiluminance}

We have pointed out that at equiluminance, at least some aspects of form perception are preserved, but that there is a reduction in acuity by a factor of 2 or 3 and a tendency for images to fade with fixation. Here we ask what other features of an image deteriorate at equiluminance, and what, if anything, they have in common.

An early attempt to address this question was made by 
Lehmann (1904), who generated figures of one color on a background of a different color and varied the luminance ratio continuously through equiluminance. He found that at equiluminance, several illusions (Fig. 27), the Münsterberg, the Poggendorff, and the Zöllner, all broke down: "So verschwindet die Täuschung vollstandig." He, and later Liebmann (1926), attempted to explain these illusions in terms of the phenomenon of "irradiation," namely that dark-light edges appear translated in the direction of the dark side (Helmholtz, 1910).

We repeated some of these experiments and found that many of the illusions in Figure 27 indeed lost their illusory distortions at some ratio of green/red brightness. This was so even if we consciously moved our eyes and prevented the image from fading. Illusions that seem to be based on the distortion of angles were dramatically reduced or entirely eliminated. The lines in Zöllner's illusion and in Hering's illusion usually appear distorted, but at equiluminance they suddenly looked perfectly straight and parallel (as indecd thcy are). With the Hering illusion, when we varied the ratio of red to green, say increasing the green, the illusory bowing of the lines diminished until they looked perfectly straight at equiluminance; the impression of straightening was so strong that we found ourselves expecting the lines to bow in the opposite direction as we continued to increase the green past equiluminance, and were surprised that they began to bow out again. In the Poggendorff illusion a straight line obliquely intersected by an occlusion acquires an illusory offset; at equiluminance the 2 parts appear collinear. Again, as we varied the ratio of red to green through equiluminance, the sensation that the lines were moving towards alignment was so strong that we found ourselves expecting them to continue moving past alignment to distortion in the other direction. (Of course they did not; on both sides of equiluminance, the illusory misalignment is in the same direction.)

Illusions involving size distortion also fail or become much less compelling at equiluminance. In the Müller-Lyer illusion, the disparity in apparent lengths of the 2 line segments was found by Lehmann (1904) to diminish but not to disappear, and this was also our impression. In the Ponzo illusion, converging lines or railroad tracks make the upper of the 2 test lines appear longer; at equiluminance they appear to be the same length. At the same time, the tracks no longer appear to recede into the distance, but look more like slanted lines in the plane of the screen.

We found that illusions that disappeared at equiluminance likewise broke down for luminance-contrast figures when the contrast was reduced below $5 \%$.

\section{Loss of depth from perspective at equiluminance}

Many of these illusions can be thought of as distortions arising from misleading nonstereoscopic depth cues, and, since depth from stereopsis disappears at equiluminance, we asked whether depth from other cues might also fail. An important nonstereoscopic cue to depth is perspective, which, indeed, also seems to break down at equiluminance: each of the drawings shown in Figure 28, for example, ceased at equiluminance to look like a surface receding into the distance, but instead simply looked like a vertical flat surface more finely textured towards the top. For some illusions, for example the Ponzo, the relation to perspective is obvious. For the Hering illusion, the radiating lines can be thought of as defining a vanishing point and creating a tunnel-like perspective receding into the distance; at equilu-

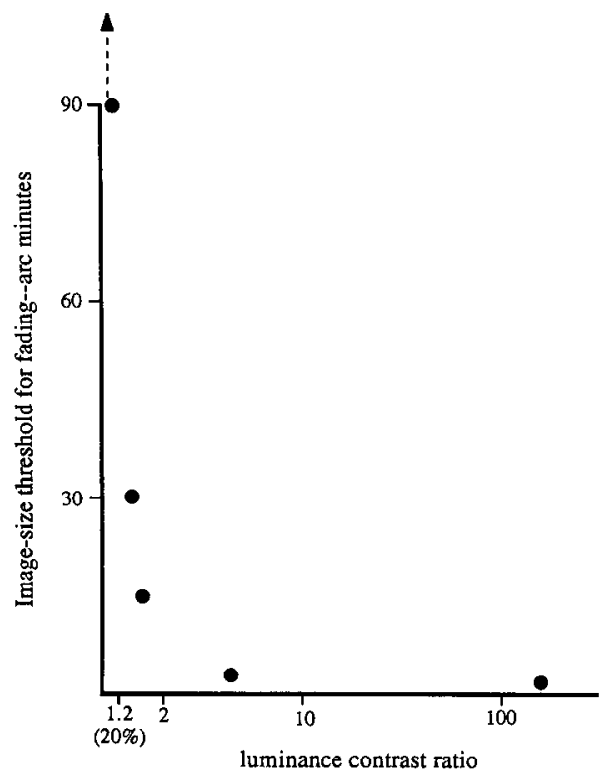

Figure 26. Fading of achromatic spots as a function of luminance contrast. We measured the threshold size spot that would fade, at $2^{\circ}$ eccentricity during voluntary fixation, as a function of the luminance contrast of the gray spot with the gray background. For a given contrast we determined the smallest-size spot for which fading occurred. Below $20 \%$ contrast, even very large spots would vanish, and, as contrast increased, up to well beyond $100 \%$, the maximum-size spot that still vanished with fixation continued to decrease.

minance they simply look like flat radial lines. In this interpretation, both the Ponzo and the Hering illusions would involve size constancy: cues in the drawing suggest depth by perspective, so that 2 objccts that in the drawing are actually the same size appear different in size because the perspective cues make one seem farther away. (In real 3-dimensional space, if 2 objects are at different distances from the observer, the object farther away must be larger in order for them to subtend the same retinal angle.) Thus in both these illusions the primary defect at equiluminance could be loss of depth from perspective, and the disappearance of the distortion would follow from the loss of the perception of depth. Other classical illusions that have been attributed to distortion by perspective cues are the Müller-Lyer (Gregory, 1963) and the Poggendorff (Gillam, 1980) (see Fig. $27 f$ ), and their breakdown at equiluminance could likewise be secondary to loss of depth from perspective.

A lower-level, less cognitive interpretation for these illusions, as well as for the perception of depth from perspective cues, is that small angles are automatically perceived as larger than they really are (Berliner and Berliner, 1948; Wallace, 1966; Bouma and Andriessen, 1970; Lennie, 1971). This overestimation of small angles has been attributed to "irradiation" (Lehmann, 1904; Helmholtz, 1910) or to mutual inhibition between cells activated by lines of similar orientation (Blakemore et al., 1970). The use of such interactions in building up perspective seems plausible because, by using such simple operations, converging lines might automatically be interpreted as being parallel but receding into the distance, and lines at comparatively less acute angles would be seen as diverging. Early stages of the visual subsystem involved in analyzing depth might use such operations to begin to deal automatically with the problem of interpreting 3 dimensions from a 2 -dimensional retinal image. As 
a

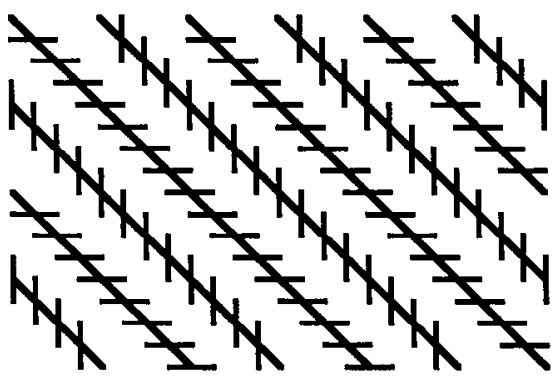

Zöllner

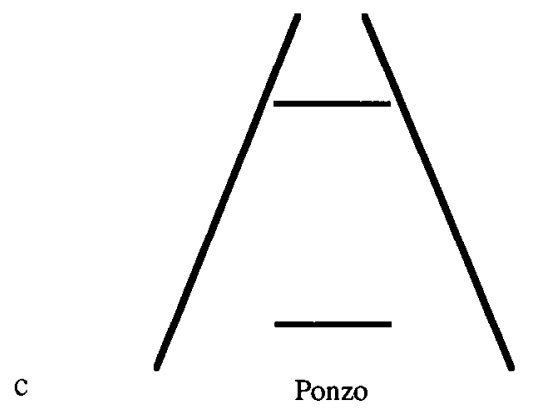

Figure 27. Various illusions made as $\mathrm{red} / \mathrm{green}$ displays; in each case the illusory distortion seen here disappears at equiluminance. $a$, Zöllner (1860): in the figure the long lines do not look parallel, but at equiluminance they do. $b$, Hering (1861): at equiluminance the horizontal lines appcar straight. $c$, Ponzo (1913) and $d$, Müller-Lyer (1894): at equiluminance the horizontal lines appear to be closer to the same length. $e$, Poggendorff (Delboef, 1893): at equiluminance the illusory offset of the oblique line disappears. $f$, Depth explanation for the Poggendorff illusion (Gillam, 1980): $a b$ and $c d$ are collinear. but in the 3-dimensional space portrayed they would not be. These illusions break down at equiluminance but persist at low luminance contrasts. They can also be seen to lose their illusory distortion during voluntary fixation.
Ponzo

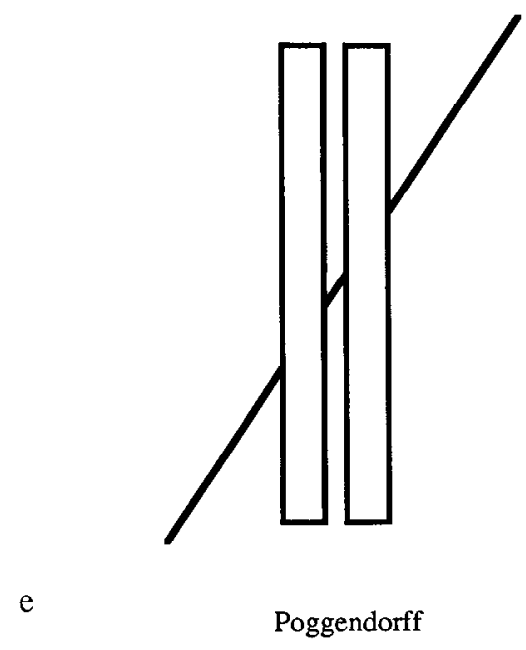

e

Poggendorff

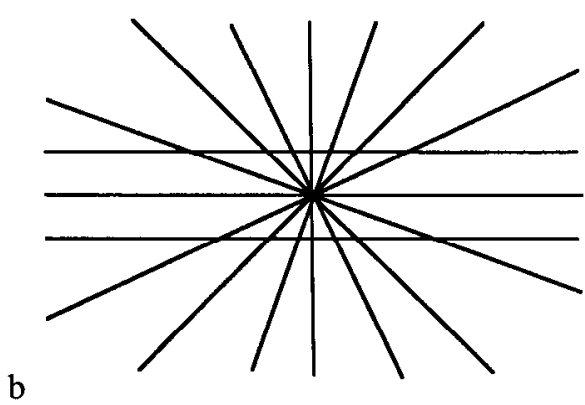

Hering
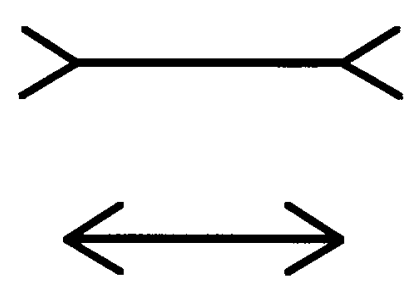

d

Müller-Lyer

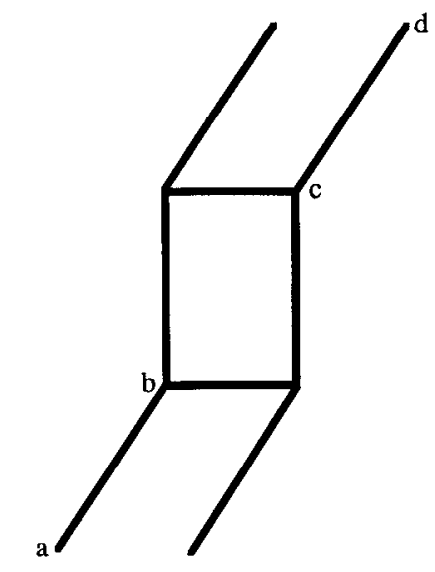

f Depth explanation for Poggendorff illusion described below, the loss of tilt induction at equiluminance suggests that it is the magno system alone that might perform such opcrations.

With most of these illusions we have the peculiar and striking fact that all of the lines and shapes in the figure are perfectly clear at equiluminance; it is only the distortion that disappears. No matter what the explanation or level of explanation for these illusions and their breakdown at equiluminance, all of them have in common their effect on comparisons between different parts of the visual field, and it is tempting to speculate that they all involve errors in processes that are involved in correlating 2-dimensional information with a 3-dimensional world.

\section{Loss of tilt induction at equiluminance}

If a test region of vertical stripes is surrounded by stripes of a slightly different orientation, the test orientation is misperceived as being in the direction away from the inducing stripes, as seen in the Zöllner illusion (Fig. 27) and in the pattern shown in Figure 29. For the pattern in Figurc 29, when we made either the test stripes or the inducing stripes, or both, equiluminant red and green, the induced tilt away from vertical in the central circle disappeared and the central stripes appeared vertical, as indeed they are. This result suggests that it is only the magno system that has inhibitory interactions between different orientations, and, if such interactions underlie the perception of depth from perspective, it might explain the dependence of perspective on luminance-contrast information. Tyler (1975) found that a random-dot stereograting with no monocular grating patterns could produce a tilt aftereffect; if, as we have suggested, the magno system is solely responsible for carrying stereo information, then this result would also be consistent with the idea that the magno system is responsible for interactions between different orientations. 

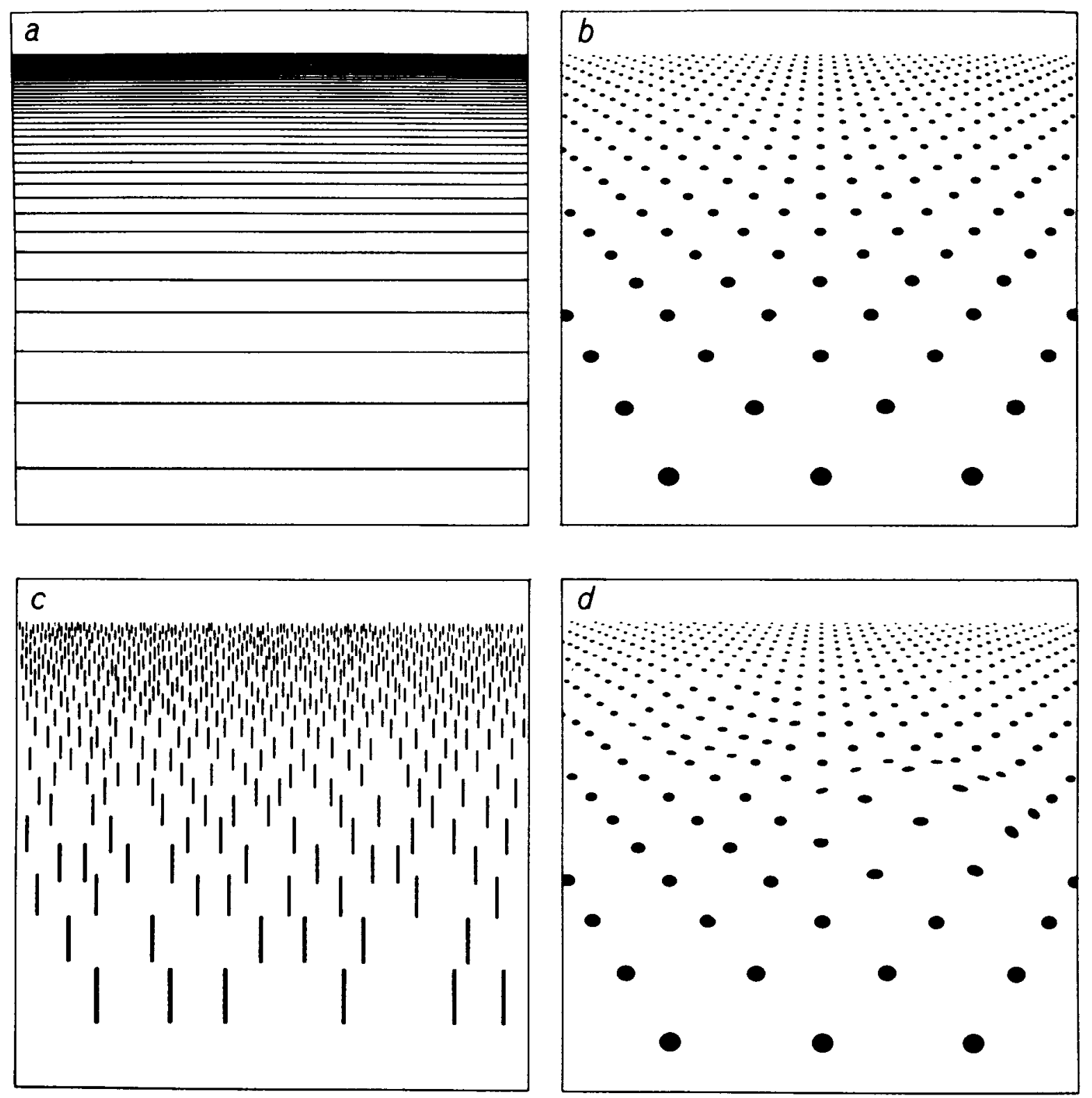

Figure 28. Loss of depth from perspective at equiluminance. $a-d$, The impression of depth that each of these gives becomes weaker or vanishes altogether when the lines are made green on an equiluminant red background, or the reverse (from Neisser, 1968). The impression of depth becomes weaker during fixation as well.

The loss at equiluminance of depth from both stereopsis and perspective raised the intriguing possibility that still other cues for depth might depend solely on luminance contrast, and we therefore tried to test as many of these as possible.

\section{Loss of depth from shading at equiluminance}

Since information from shadowing consists, under most lighting situations, entirely of luminance-contrast information, a system whose function is to generate shape from shading might just as well be achromatic. While this is intuitively plausible, one cannot simply assume it is so, since interpreting shading could be just one function of a more general system. We will describe some experiments that suggest that the analysis of depth and of 3-dimensional shape from shading is distinguishable by its achromaticity from the analysis of 2-dimensional shape.

In Figure 30, the pattern of yellow dots on a green background gives the sensation of a 3-dimensional object. When the dots are darker or lighter than the background, the perceived shape is the same, as long as there is sufficient luminance contrast of the dots with the background. At equiluminance, however (which we hope has been approximated for some readers in the middle panel), the shape was no longer easily recognizable. Even though it is almost impossible to achieve equiluminance for any one person without being able to adjust the color ratio continuously, we have attempted it here with a color print because, for us, the loss of depth in this situation occurred over a remarkably wide range of yellow/green intensities. (For stereopsis or motion, for example, the setting was much more critical.)

\section{Shape from texture}

A similar loss of depth at equiluminance was seen with the pattern shown in Figure 31 (Riley, 1964). The wavy pattern 
Figure 29. Display for determining the strength of induced tilt effect. The central stripes are vertical, but appear tilted away from the slant of the surrounding stripes, except at equiluminance, when there is little or no effect of the surrounding stripes.

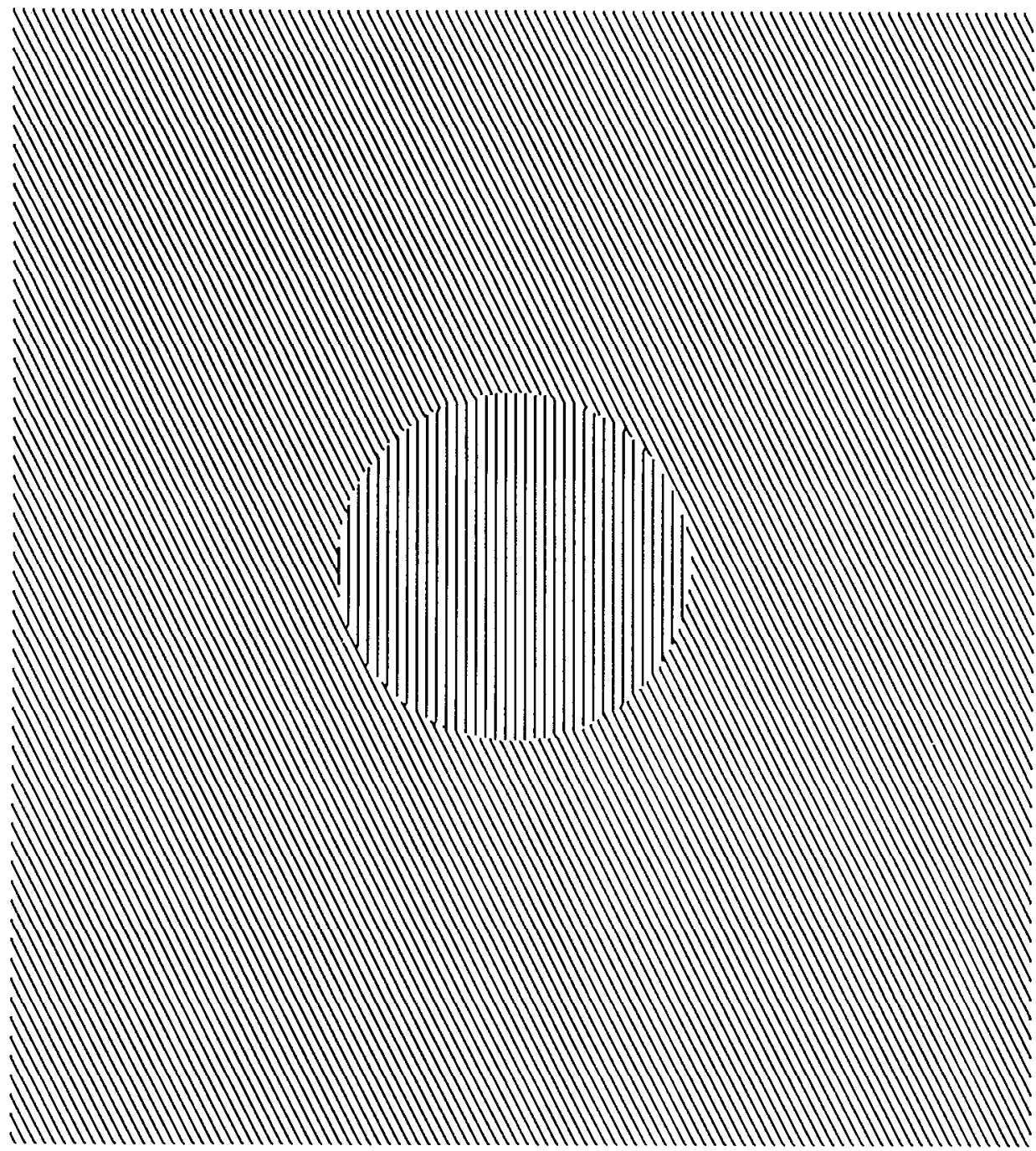

gives a sensation of depth and motion: the depth perception is bistable, in that any region of curvature can be seen as either a crest or a trough, and as the perceived depth reverses it produces a sensation of undulating movement. When we made this pattern equiluminant, the sensation of depth and movement was greatly reduced, even though the wavy parallel lines were still clearly seen. If we continuously varied the ratio of red to green, we usually had the sensation that the depth reversed at cquiluminance, so that whatever had been perceived as a crest suddenly became a trough.

The depth in this figure is not generated by shading, as one can verify by defocusing the image and seeing that it becomes uniformly gray, with no average luminance difference contributing to the perceived depth. The depth here is generated by contour lines, which are an artistic exaggeration of the normal anisotropy of texture that arises as a result of oblique viewing angles. (Tilting a textured surface increases the proportion of contours parallel to the surface normal.) This result suggests that shape from texture is also mediated by the magno system, so we would predict that depth from texture, like movement and stereopsis, should disappear at very high spatial frequencies. Indeed, if one views this figure at very close distances, the sensation of depth is most vivid, and at distances at which the spacing of the lines is above $8-10$ cycles/deg, the sensation of depth diminishes considerably, even though the wavy lines are still discernible.

\section{Depth from movement}

Another strong cue for depth can be provided by movement, in particular the relative movements of objects in the visual ficld duc to the motion either of the objects themselves or of the observer (Helmholtz, 1910; Gibson, 1950). We asked if the sensation of depth due to movement parallax or to relative motion disappears at equiluminance. Figure 32 shows a pair of figures that form part of a series that we showed cinematographically. Each figure is made up of dots distributed as though they had been stuck at random on a transparent sphere and then projected onto a flat surface. In successive pictures, the dots move as though the sphere were rotated by a constant small angle. Each frame looks like a collection of dots scattered on a circle, but when the display changes every $1 / 10 \mathrm{sec}$, with some dots moving to the right and others to the left, one has at nonequiluminance a vivid sensation of a rotating 3-dimensional sphere. Both the front and back of the sphere appear to be smooth rigid surfaces, with all the dots on the surface moving 

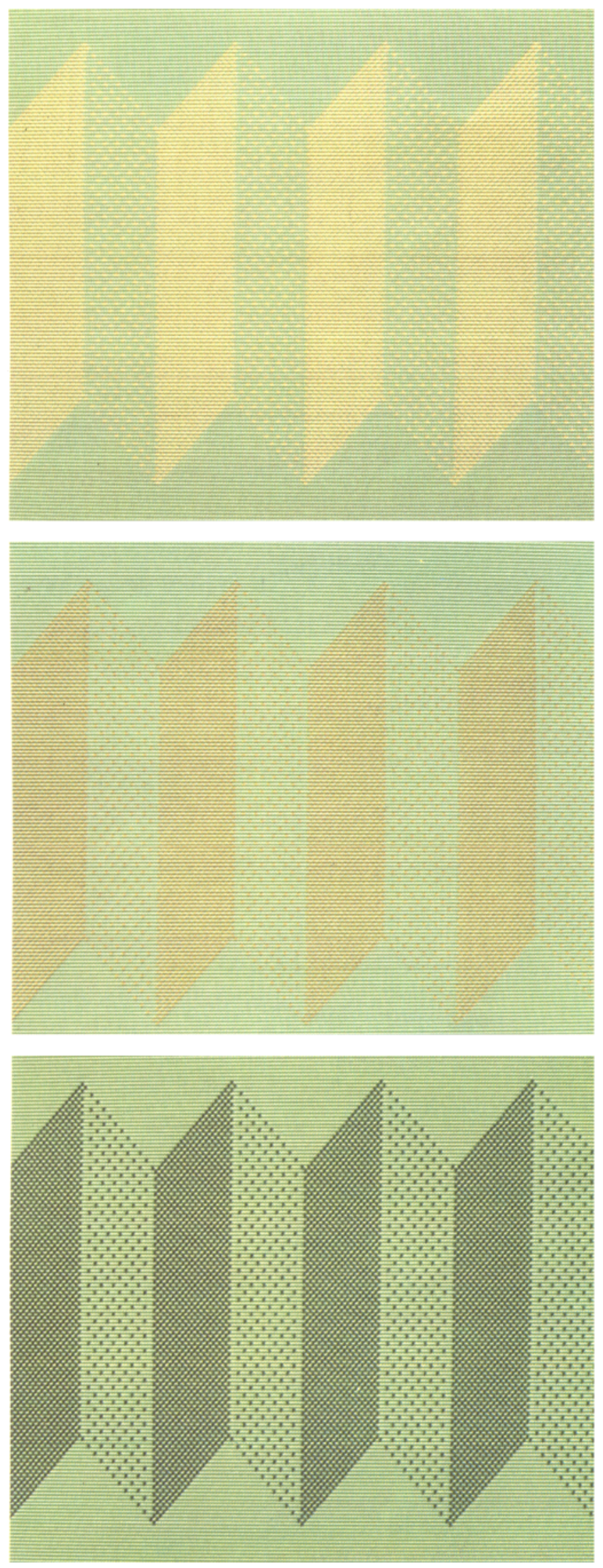

Figure 30. Loss of shape from shading at equiluminance. The concentration of dots is used here to portray shading, just as stippling is together in an organized, coherent manner. (The depth is bistable, in that the sphere can be seen to rotate in either direction, reminiscent of a Necker cube.) When the dots were made equiluminant with the background, all sensation that the object was a sphere in depth was lost, along with the sensation of a smooth surface moving rigidly. The coherence of the dots' movements ceased and they all seemed to be moving and twitching independently in a flat circular cluster, no longer only horizontally, but in all directions.

When we made the dots gray on a gray background, we required only $10 \%$ luminance contrast to see the sphere in depth. The movement of the dots on the rotating sphere seems to have something in common with the multiple, bistable apparent movement squares in Figure 7, in that both phenomena require luminance contrast for coherence of movement in different parts of the visual field.

Next we examined depth from movement parallax, that is, the sensation of depth produced by the relative movement of objects in the visual field as a result of the observer's own motion. We made a display that was coupled to the observer's head position so that side-to-side head movement resulted in horizontal movement of a vertical bar (Fig. 33). At nonequiluminance the bar appeared to be at a different depth than the stationary reference bars. At equiluminance, they all appeared to be in the same depth plane even though the shifts in relative position were easily seen.

Several other displays that produce a strong illusory sensation of movement in depth, which in each case disappears at equiluminance, are shown in Figure 34. A rotating logarithmic (equiangular) spiral (Fig. 34a), when rotated clockwise, appears to loom towards the observer; counterclockwise, it appears to recede (Steinhaus, 1938). When spiral and background were made equiluminant, the approaching or receding sensation disappeared, and the spiral seemed simply to spin, as in fact it did. In Figure 34, $b$ and $c$, we show 2 kinetic depth illusions, such as those described by Musatti (1924); when these disks are rotated slowly (about 10-20 rpm), the ellipse in Figure $34 b$ begins to look like a circle rolling around the center at an angle in depth, and the circles in Figure $34 c$ seem to lie on an eccentric cone; at equiluminance, both figures appeared flat.

\section{Depth from occlusion and illusory contours}

Occlusion is a powerful cue both for depth and for organizing objects in the visual field. In Figure 35, a square is seen in front of a partly occluded circle. If the square was red and the visible part of the circle equiluminant green, the square no longer seemed to be clearly in front of the circle, and the green area no longer looked like an occluded circle, but like a Pac Man figure biting the square, with both in the same depth plane.

Figure 36 shows 3 drawings that produce the sensation of borders, or contrast, where there is no real border (Schumann, 1900; Kanizsa, 1955, 1974, 1979). Many such illusory borders have been interpreted in terms of occlusion-for example, a triangle occluding 3 circles (Fig. $36 a$ ), a circle occluding spokes

used by artists. In the top and bottom panels the stippling is not equiluminant with the background and gives an impression of a 3-dimensional shape. When the dots and background are close to equiluminant, the impression of depth becomes far less compelling. 


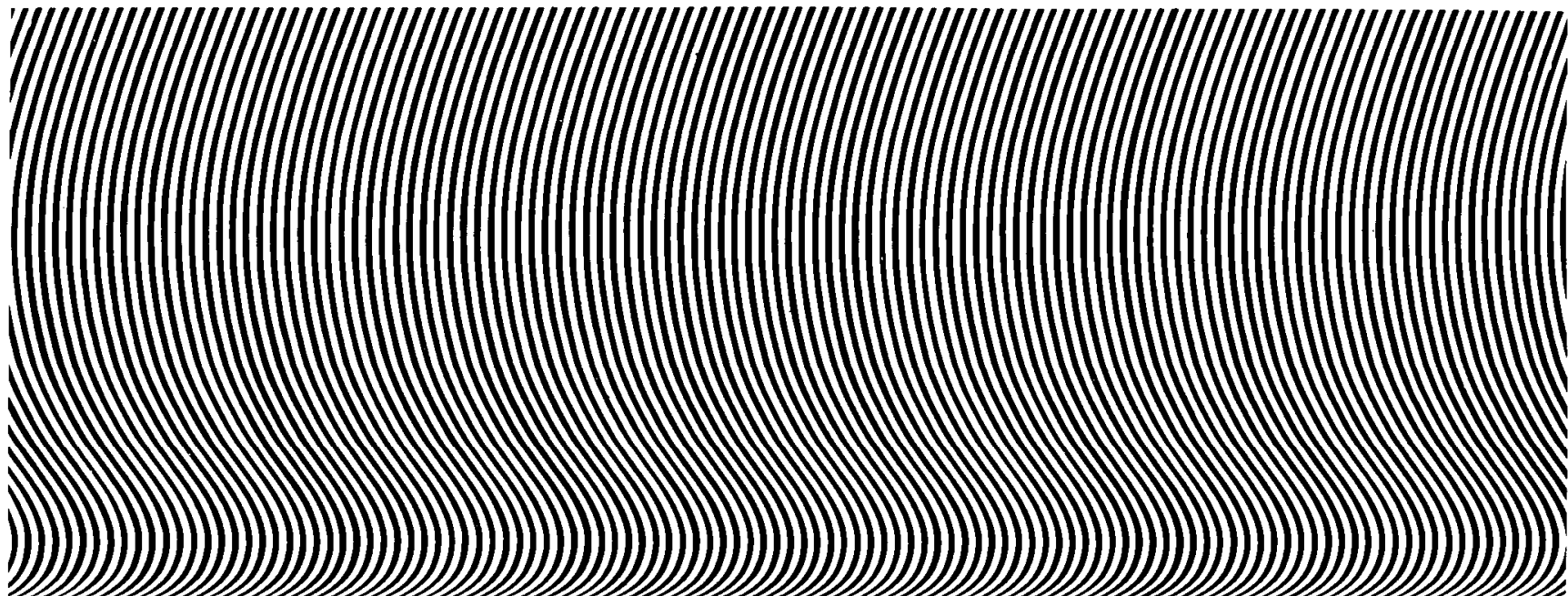

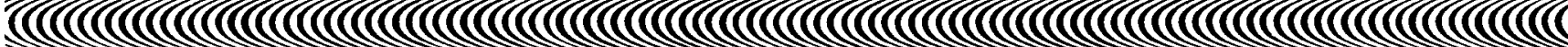

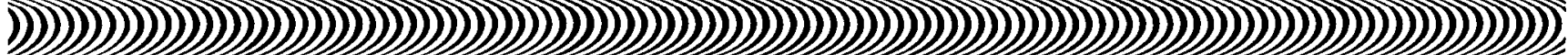

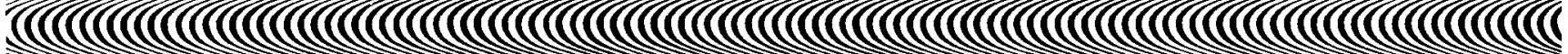

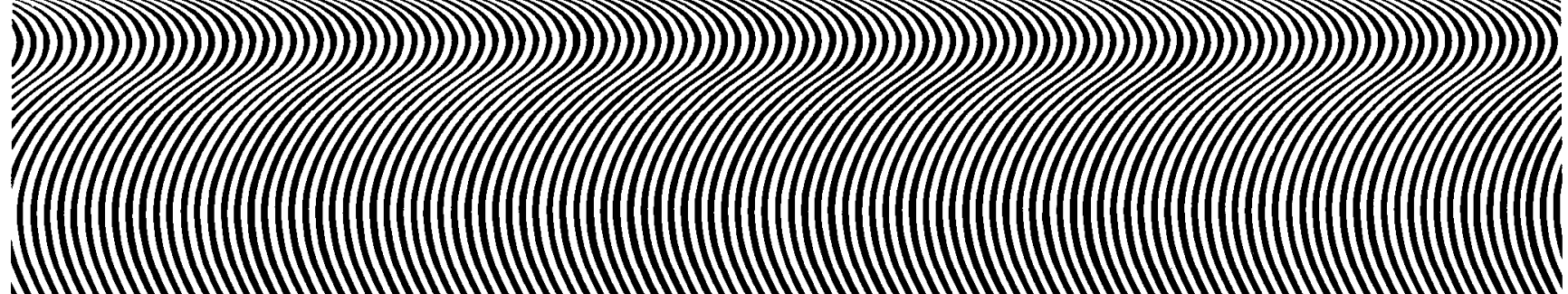

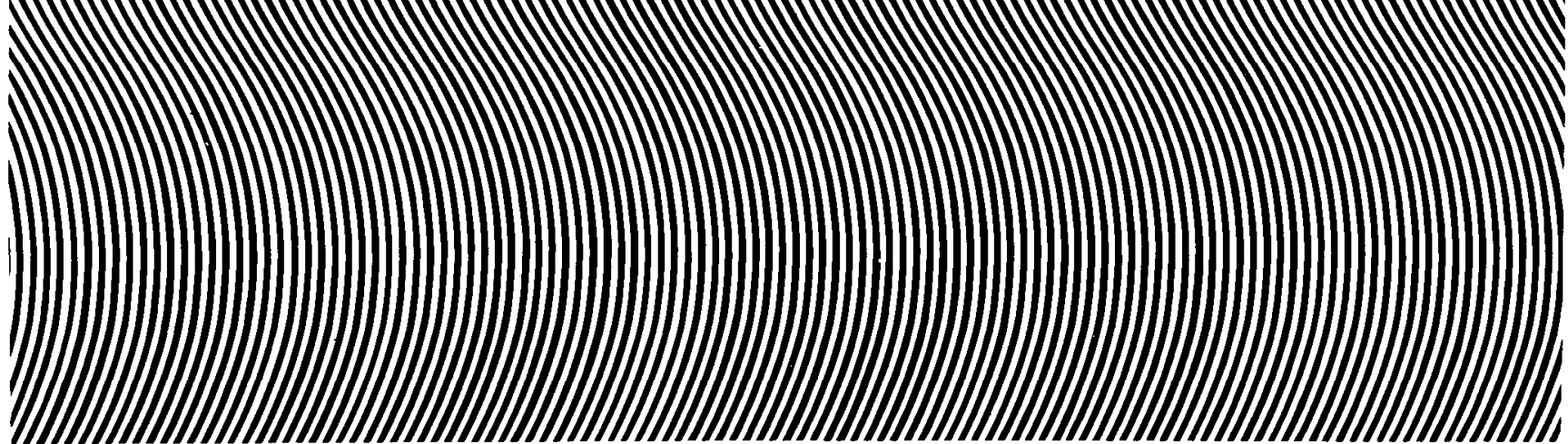

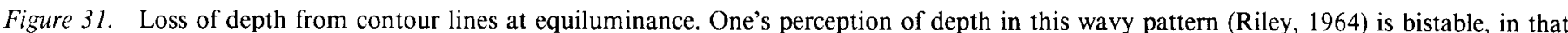

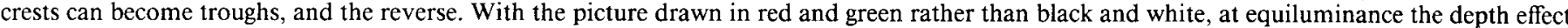

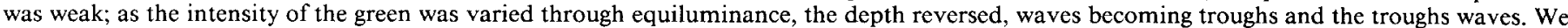

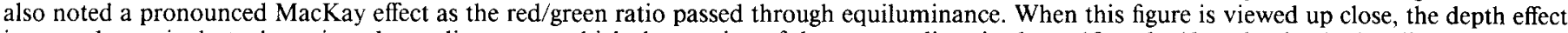
is most dramatic, but when viewed at a distance at which the spacing of the contour lines is above 10 cycles/deg, the depth also disappears.

(Fig. 36b), or one piece of lined paper covering another (Fig. $36 c$ ). Such figures suggest a strong tendency for the visual system to join or link edges or points that are collinear or lie on a common curve.

Illusory borders look different from real borders: they are sharp, but fragile. They have no independent existence; the triangle requires the Pac Men, and the circle the spokes. A striking difference in behavior between illusory and real borders is that when the patterns were made of equiluminant red and green, the figures defined by the illusory borders-for example, the triangle, the disk, and the edge in Figure 36-completely dis- appeared, but the objects defined by real borders - the Pac Men, the spokes, and the stripes-appeared almost as distinct as they did at nonequiluminance, though they no longer seemed to be occluded by something; for example, at equiluminance, Figure $36 a$ looked like 3 circles with triangular cutouts rather than like 3 entire circles partly occluded.

Why should the real and illusory parts behave differently at equiluminance? It is as if 2 independent processes were carrying information about the real edges, one capable of discriminating form using color contrast, without the need of luminance contrast, and a second, achromatic, process that requires luminance 

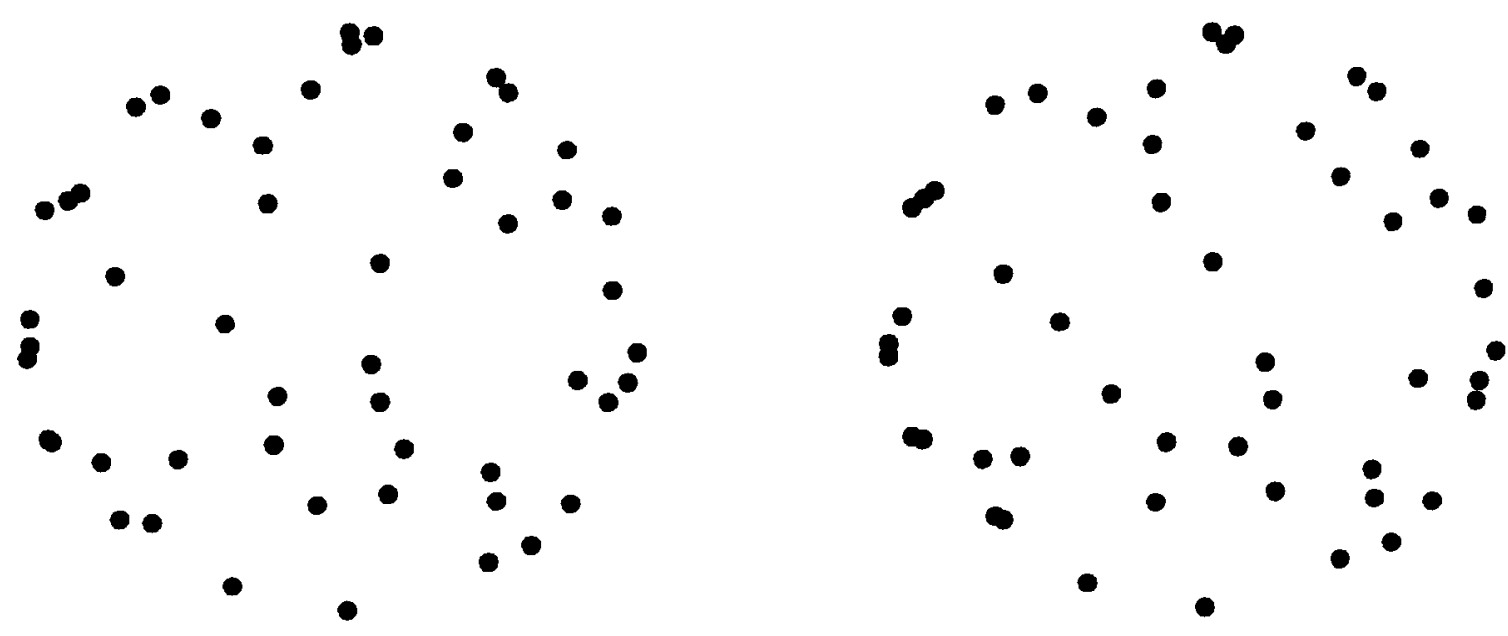

Figure 32. Loss of depth from motion at equiluminance. If one views cinematographically an appropriate succession of such figures, beginning with the 2 illustrated, the impression is of a sphere rotating. If the dots are made green on a red background, or the reverse, one sees a 3-dimensional sphere rotating smoothly, except over a narrow red/green range, when all sensation of depth disappears and the dots seem to move incoherently. A sensation of depth without the rotation can be seen here by viewing these 2 images stereoscopically.

contrast and whose function it is to join together collinear real borders and produce the illusory ones. Away from equiluminance, both systems presumably see the Pac Men, but only the achromatic system sees the triangle. Because of this loss at equiluminance, we would postulate that it is the magno system that generates the illusory border by joining together collinear real edges, and that the parvo system does not possess such a tendency to join spatially separate edges. (This difference may be due to the fact that there are many end-stopped cells in the parvo system but not in the magno system.)

If the magno system is responsible for generating illusory contours, then we would predict that illusory borders should be perceived at low luminance contrasts, and indeed we found that we could see illusory contours very clearly at $10 \%$ contrast and even at $5 \%$ contrast, although then they were fuzzy. Brussel et al. (1977) previously reported that luminance differences but not color differences were necessary for the perception of illusory borders. Illusory borders, as in Figure $36 c$, also disappear when the lines are finer than 10 cycles $/$ deg.

Illusory contours can be much more vivid when they are combined with other cues such as stereopsis or movement (Ramachandran, 1985; Ramachandran and Cavanagh, 1985); such a synergism between the linking of collinear edges with motion or stereopsis may be possible because they are all three performed by the same system.

The fact that illusory borders disappear at equiluminance would seem to rule out a high-level cognitive explanation for them, such as an assumption of an occlusion.

\section{Figure-ground}

A particular border in a visual scene can be caused by the edge of an actual object in front of a background, by an edge of one
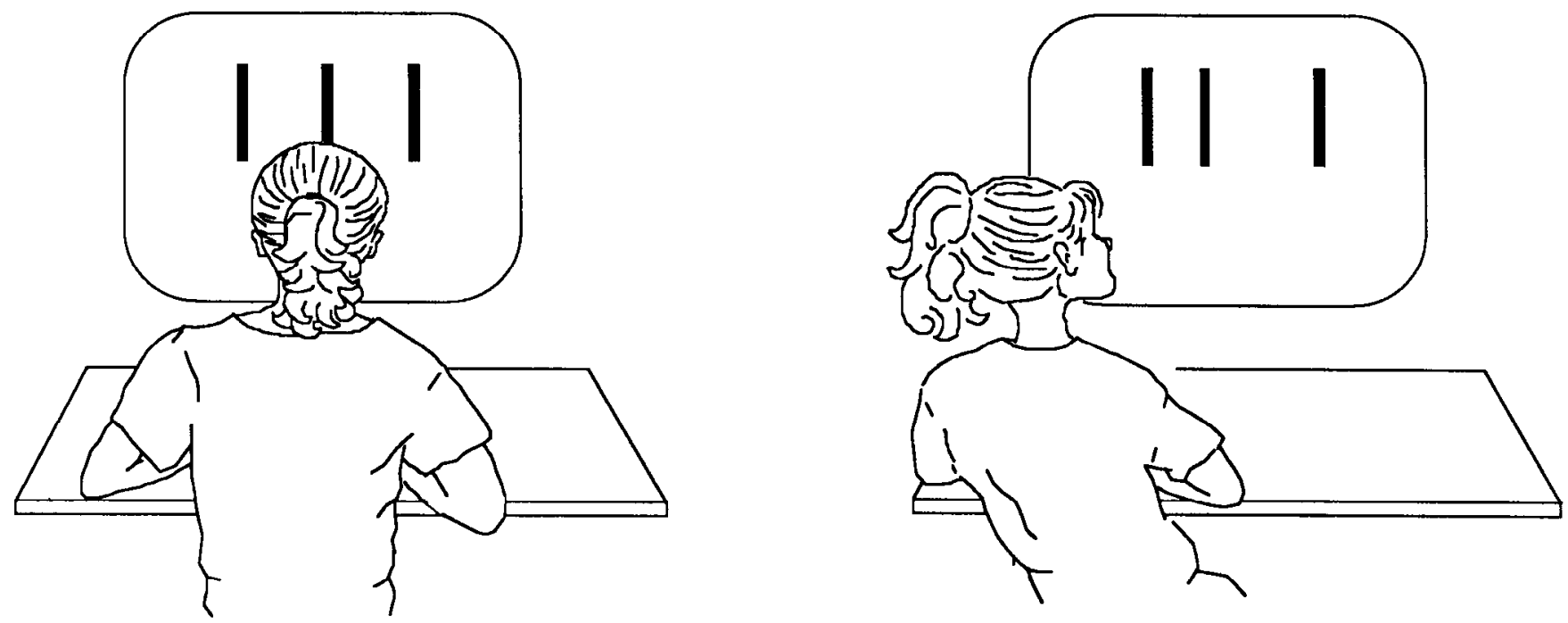

Figure 33. Loss of depth from parallax at equiluminance. The observer holds a mouth bar (not shown). As the head moves from side to side, the bar turns a potentiometer, causing the middle rectangle on the monitor to move. The result is an illusion of depth from parallax; when the middle bar moves as indicated, it appears to lie behind the others. With green rectangles on a red background, or the reverse, at equiluminance the sensation of depth is lost. 


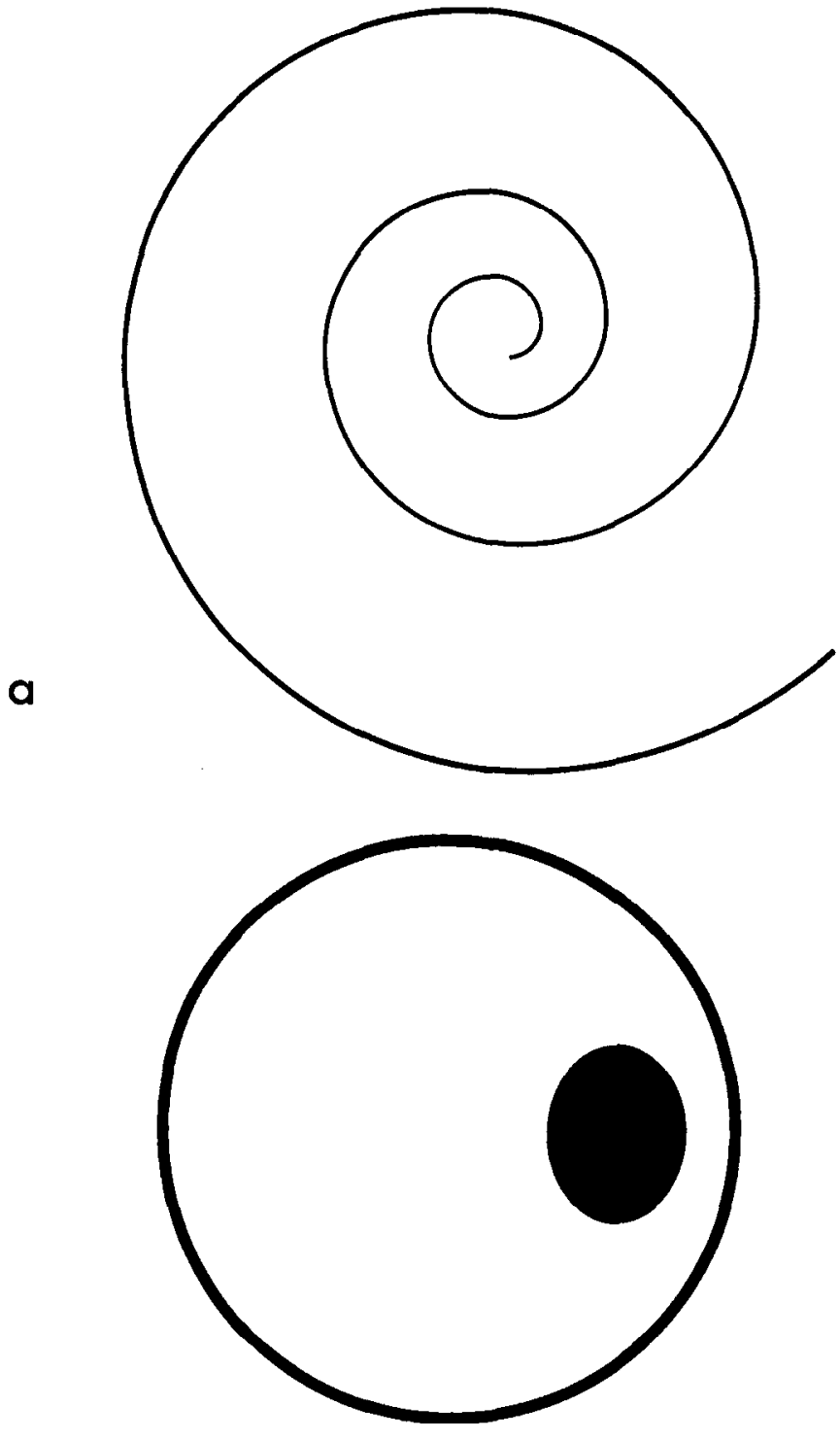

b.

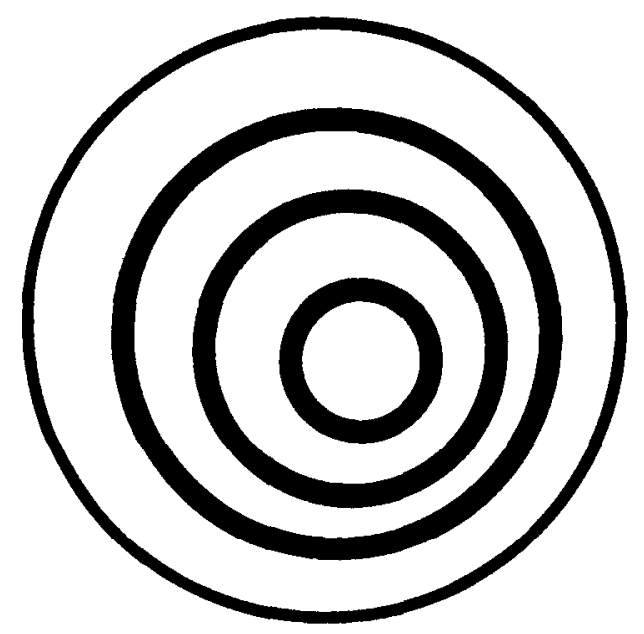

C. object occluding another object, or by a shadow or bend within a single object, and so on. The Gestalt psychologists analyzed the cues that are used to decipher the organization of the objects in a visual scene and to segregate objects from background. The differentiation of figure from background seems to depend on many of the cues that break down at equiluminance, such as occlusion, stereopsis, and relative movement. Liebmann (1926) described the difficulty in distinguishing figure from background at equiluminance, and Koffka (1935) pointed out that luminance differences are strikingly more important than color differences: "Thus two greys which look very similar will give a perfectly stable organization if one is used for the figure and the other for the ground, whereas a deeply saturated blue and a grey of the same luminosity which look very different indeed will produce practically no such organization." A popular illustration of the problem of deciding between figure and ground is the vase/face shown in Figure 37a (Rubin, 1915). At nonequiluminance, the percept is bistable, so that one sees either the face or the vase, but usually not both at the same time. The same is true of Freud and friend (Fig. 37b). With the figures redrawn in red and green, varying the red-to-green ratio produced a reversal in the percept at cquiluminance; whichever had been the figure suddenly bccame the background and vice versa. The reversal was reminiscent of what we had seen with the waves and troughs in Figure 31 . With the figures held at equiluminance, the 2 percepts alternated more rapidly, and one could often see both vase and face, or Freud and friend at the same time.

\section{A subsystem for spatial organization: linking}

It was the loss of stereopsis at equiluminance that prompted us to examine perspective, size constancy, parallax, relative motion, shading, and occlusion, because we wondered whether all kinds of information about depth might be carried by a colorblind system. Although we have not exhausted the list of possible depth cues, or exhaustively examined any one of them, our overall impression is that they all deteriorate dramatically at equiluminance. Since it is clear that not all visual abilities are lost at equiluminance-for example, shape recognition, including high-resolution shape discrimination, shows very little deterioration-we wondered whether all the abilities that are lost at equiluminance are functionally related or part of one visual subsystem, whose functions include the perception of motion, of depth from various different types of information, and perhaps other functions involved in analyzing 3-dimensional spatial organization.

Why should all these different abilities be carried by the achromatic system and not by the chromatic system? What these different types of visual information may have in common is

Figure 34. Loss of stereokinetic depth effects at equiluminance. $a$, An equiangular (logarithmic) spiral, which increases its size on rotation without changing its shape (see Coxeter, 1969). Eadem mutata resurgo ("though changed in size I shall arise the same": Bernoulli, 1705). When it is rotated, at nonequiluminance it appears to approach or recede; at equiluminance the sensation of movement in depth disappeared. $b$, When this disk is rotated slowly on a turntable, after a few minutes the eccentric ellipse begins to look like a circle lying at a slant in depth, rolling around. When the ellipse was red on a green background (or the reverse), at equiluminance the sensation of depth vanished. $c$, When this disk is rotated, the circles seem to lie on a cone that comes out in depth towards the obscrver, and this sensation of depth also vanishcd at equiluminance. 

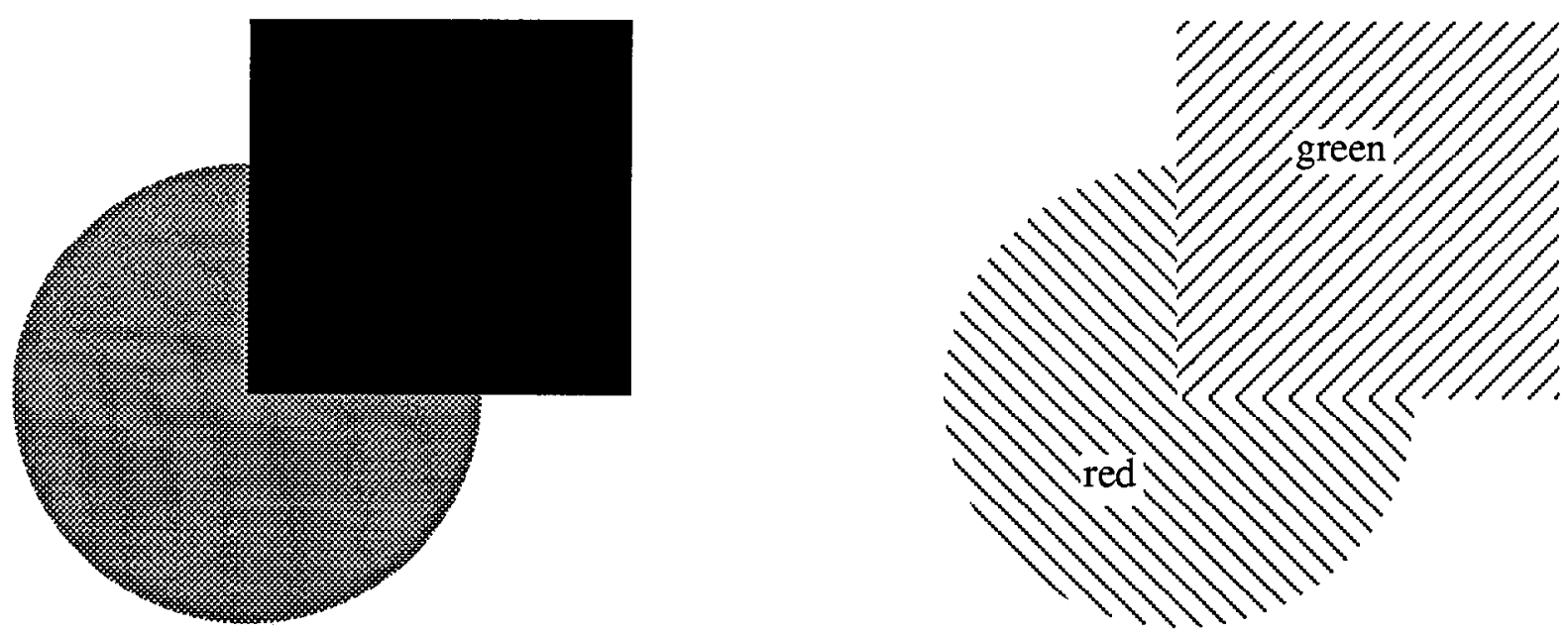

Figure 35. I oss of depth from occlusion at equiluminance. At nonequiluminance, the green square seems to be in front of and blocking off part of a red circle. At equiluminance, the figure appeared to be a square and a $3 / 4$ circle in the same plane.

that they can all be used to distinguish objects one from another and from the background, to correlate parts of the visual field that belong to the same objects, and to assign relative positions to objects in space. Rubin (1915), Wertheimer (1923), and Barlow (1981) have suggested that certain visual properties of objects are used to group together parts of an image, to integrate figures, and to separate objects from each other and from the background, properties Barlow called "linking features." These include direction and velocity of motion, depth (stereopsis, occlusion, and so on), collinearity, lightness, color, and texture. While the list is not totally congruent with our list of itcms that fail at equiluminance, color being an important exception, the 2 are similar enough to be encouraging. The results described above suggest that color itself may not be a linking feature, but that it is the luminance difference usually present in a colorcontrast border that is used for linking. A simple example of the use of collinearity as a linking feature is shown in Figure 38. Here one immediately knows which lines belong to which object, even when they are separated by long distances because of occlusion by other objects. One can perceive the 3-dimensional shape of each of the objects, and how they are arranged in space. We found that at equiluminance this order broke down and we saw only a jumble of lines, instead of a pile of blocks. Like many other phenomena that break down at equiluminance, our ability to see the spatial organization in this figure was still intact at $10 \%$ contrast, so we suspect that this function is also carried by the magno system.

\section{Where versus what in higher visual areas}

Shape discrimination is possible both at low luminance contrasts and at equiluminance, so we would have to conclude that both the magno and parvo systems have some ability to discriminate form. The highest-resolution form discrimination, however, seems to be carried only by the parvo system. We have suggested that the magno system is responsible for the perception of motion and depth and for the ability to organize a scene in 3 dimensions, that is, to determine where objects are. The idea that higher visual functions may also be so subdivided, with the subdivisions carried by anatomically separate pathways, receives some support from lesion studies in mon- keys. These studies suggest that visual areas beyond area 18 can be divided into 2 separate pathways, one that seems to be important for the recognition of objects, the other for determining the spatial relationships of objects (Pohl, 1973; for review, see Mishkin et al., 1983; Van Essen and Maunsell, 1983). Spatialdiscrimination learning (remembering the location of objects) is severely affected by posterior parietal lesions and not by temporal lesions, whereas the reverse is true for object-discrimination tasks, a distinction described by Mishkin et al. (1983) as
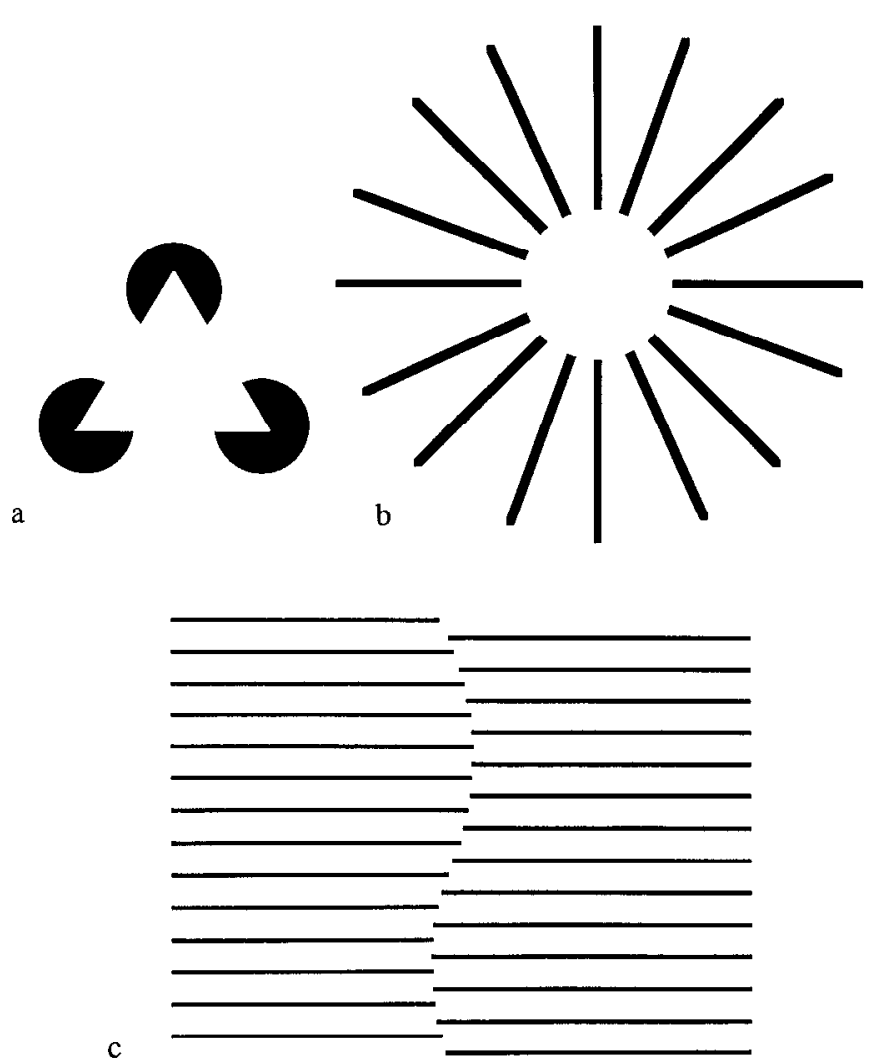

Figure 36. $a-c$, Three examples of illusory contours, all of which vanish at equiluminance, but can be seen at $5 \%$ luminance contrast. 


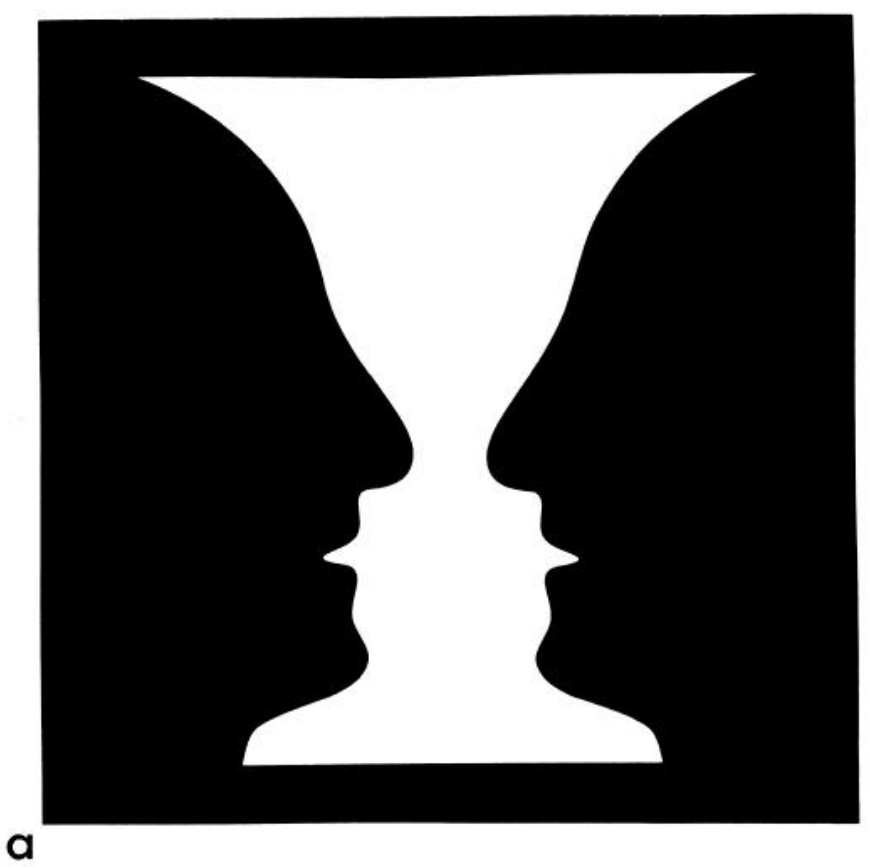

Figure 37. Loss of figure/ground discrimination at equiluminance. $a, \mathrm{Ru}-$ bin's vase and face (1915). $b$, Freud and friend. For both drawings, at nonequiluminance, only one of the 2 possible percepts is usually seen at any instant. At equiluminance, both percepts can be seen at once, and if the red/green ratio is varied, as the image passes through equiluminance, one's percept usually shifts.

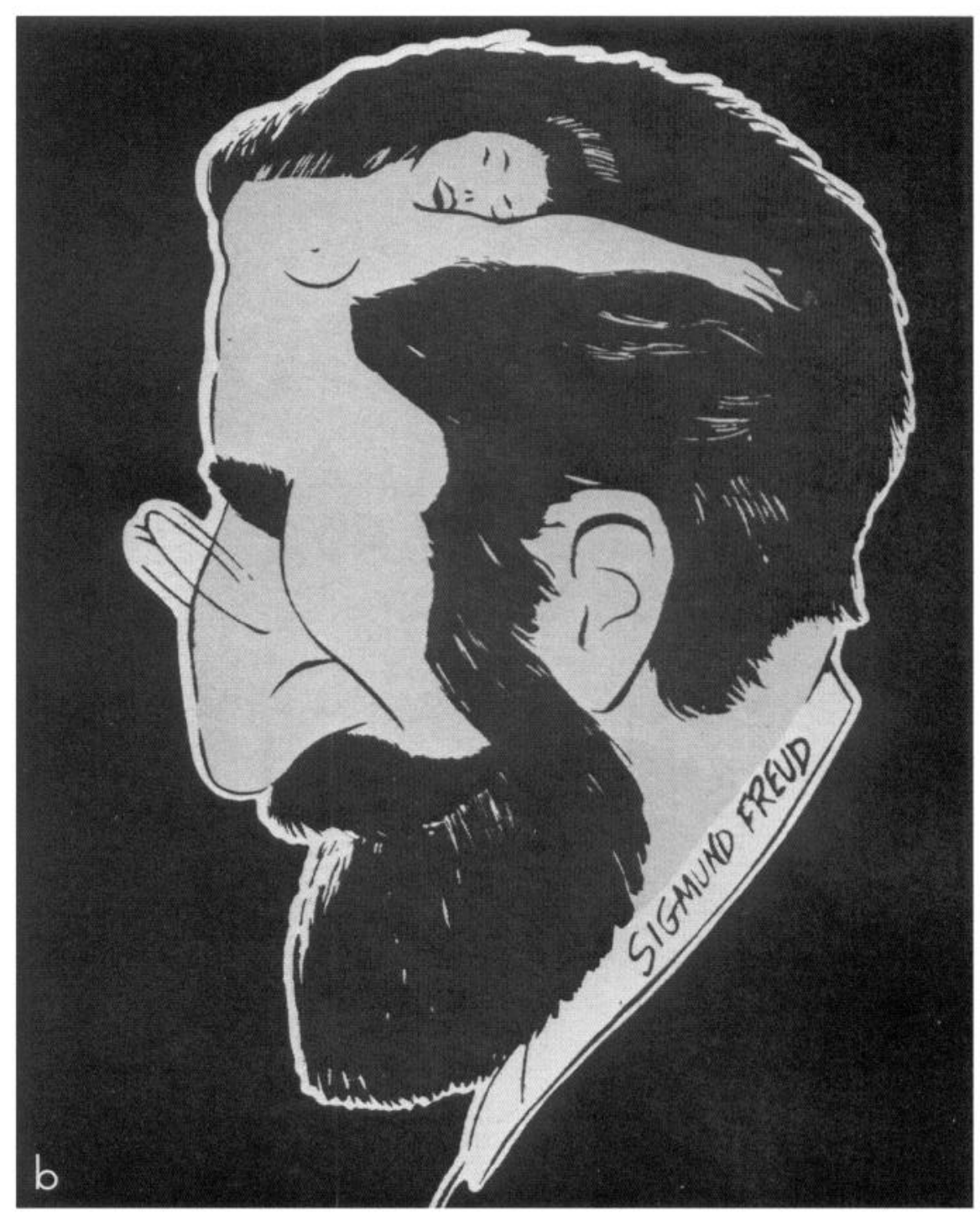




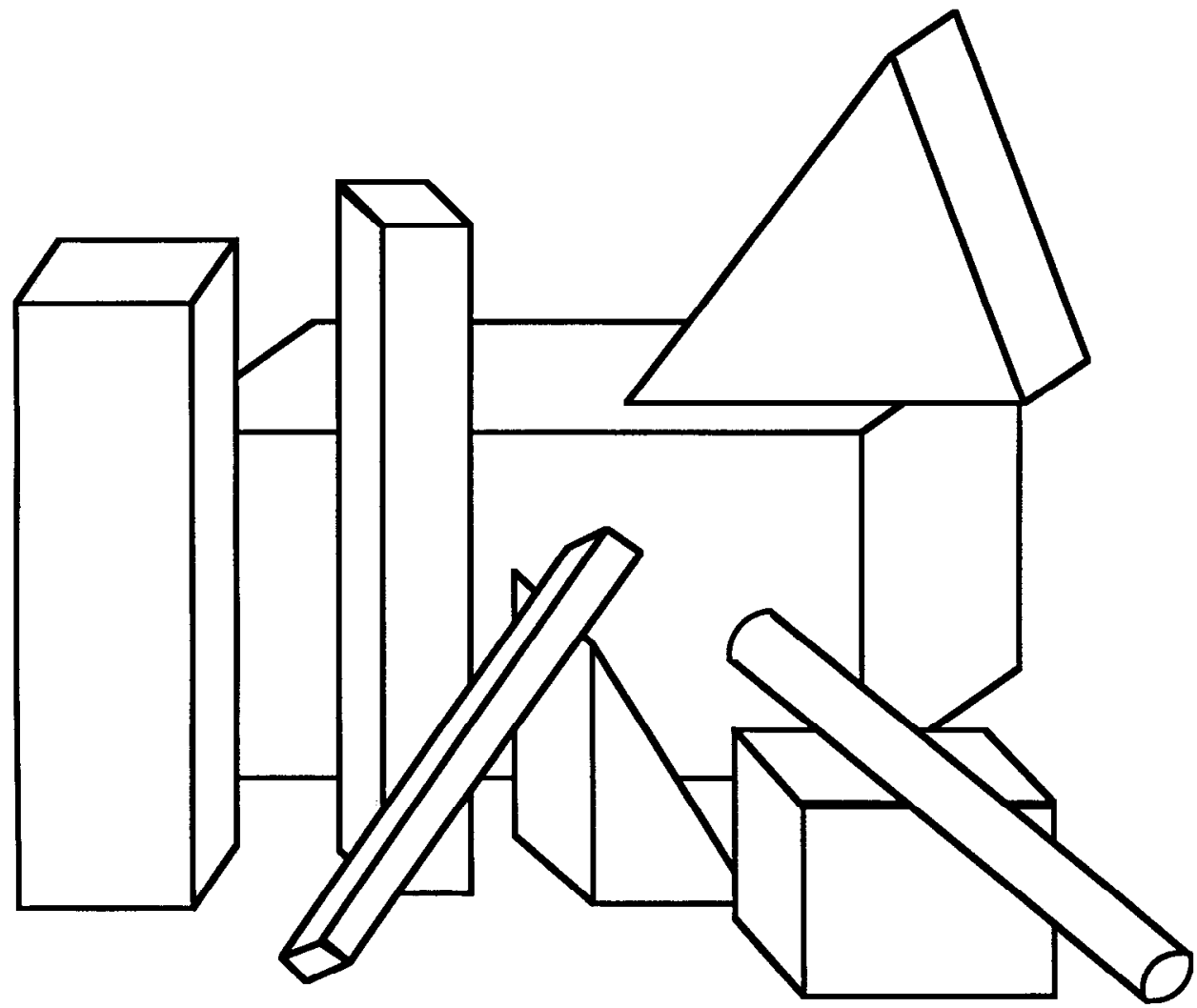

Figure 38. Pattern used to demonstrate the use of collinearity as a linking feature. When the lines were made red on a green background (or the reverse), at equiluminance the figure appeared to be just a jumble of lines, and the appearance of discrete 3-dimensional objects disappeared. where versus what. The posterior parietal region gets its input from $\mathrm{MT}$ and from visual area 3, and could thus represent stages in the magnocellular pathway beyond the thick stripes and MT. Newcombe and Russell (1969) have summarized clinical evidence for a similar dissociation of object recognition and visual spatial abilities in humans. Recently Rizzo and Damasio (1985) studied the stereoscopic depth perception of patients with focal cortical lesions. They report loss of stereo acuity with occipitoparietal lesions, but not with occipitotemporal lesions. If, as we suggest, stereopsis is solely a function of the magnocellular system, then their results give further support to the idea of a magnocellular input to the posterior parietal region. It is tempting to speculate that the psychophysical abilities that are lost at equiluminance reflect the functions of this magno-derived system.

Figure 39 shows a complex image by M. C. Escher (woodcut: Tower of Babel, 1928) that illustrates the loss at equiluminance of perspective, linking, and figure/ground discrimination.

\section{Differences in equiluminance settings for different visual tasks}

In the course of this study we examined many psychophysical phenomena at equiluminance. As we have already remarked, the green-to-red intensity ratios at which many phenomena are minimized vary with the phenomenon, with the observer, and with eccentricity. We found that although our two usual observers, ourselves, differed with all of these equiluminance settings, it was always in the same direction (D.H.H. requiring more green) and always by about the same amount. The phenomenon that was easiest to measure and that gave the most consistent and precise results was color flicker photometry, which we have adopted as a standard in Figure 40. Here we have called the green/red ratio for flicker fusion 1.0 for each of us, and the other equiluminance values are scaled accordingly. The results indicate a wide range of ratios for "equiluminance" for different functions, all requiring less green than the ratio for flicker fusion. Our 2 sets of values tend to be correlated. For a given task it made no difference whether the figure was red on a green background or the reverse.

We suspect that some of the variation in equiluminant points for different tasks may depend on the differing temporal requirements of the tasks. Cells in the visual system, particularly in the magnocellular geniculate layers, can show striking differences in responses to color as a function of stimulus size and duration: magno geniculate cells have broadband field centers that give transient responses to small spots, whereas their field surrounds give tonic responses and are often more heavily weighted by red-cone input. For such cells the spectral sensitivity varies with the temporal properties of the stimulus.

\section{Temporal limitations for various visual tasks}

By comparing thresholds for detecting modulations of gratings in which either color contrast or luminance contrast was varied as a function of the temporal frequency of the modulation, Kelly and van Norren (1977) concluded that there are 2 independent visual channels, a chromatic channel and an achromatic channel, and that the achromatic channel has a higher temporal resolution. From the physiological evidence, we would speculate that their fast achromatic channel corresponds to the magno system and the slow chromatic channel to the parvo system. Kelly and van Norren's (1977) success in using temporal differences to distinguish 2 channels suggests that such temporal differences could also be used to differentiate between other properties and functions of the 2 pathways.

The fastest rate at which one can follow an alternation between 


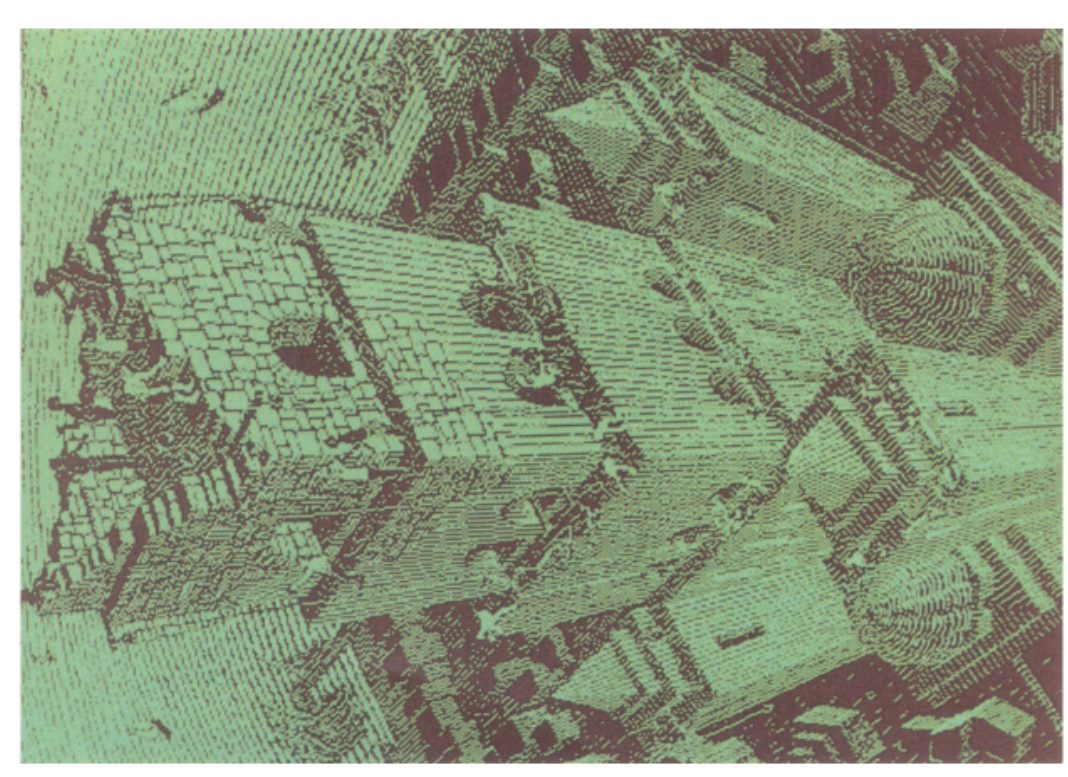

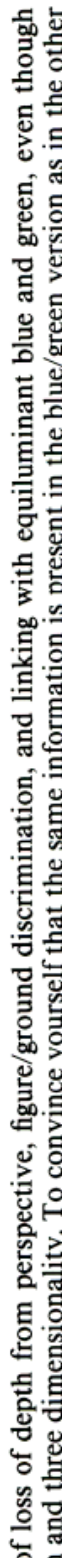
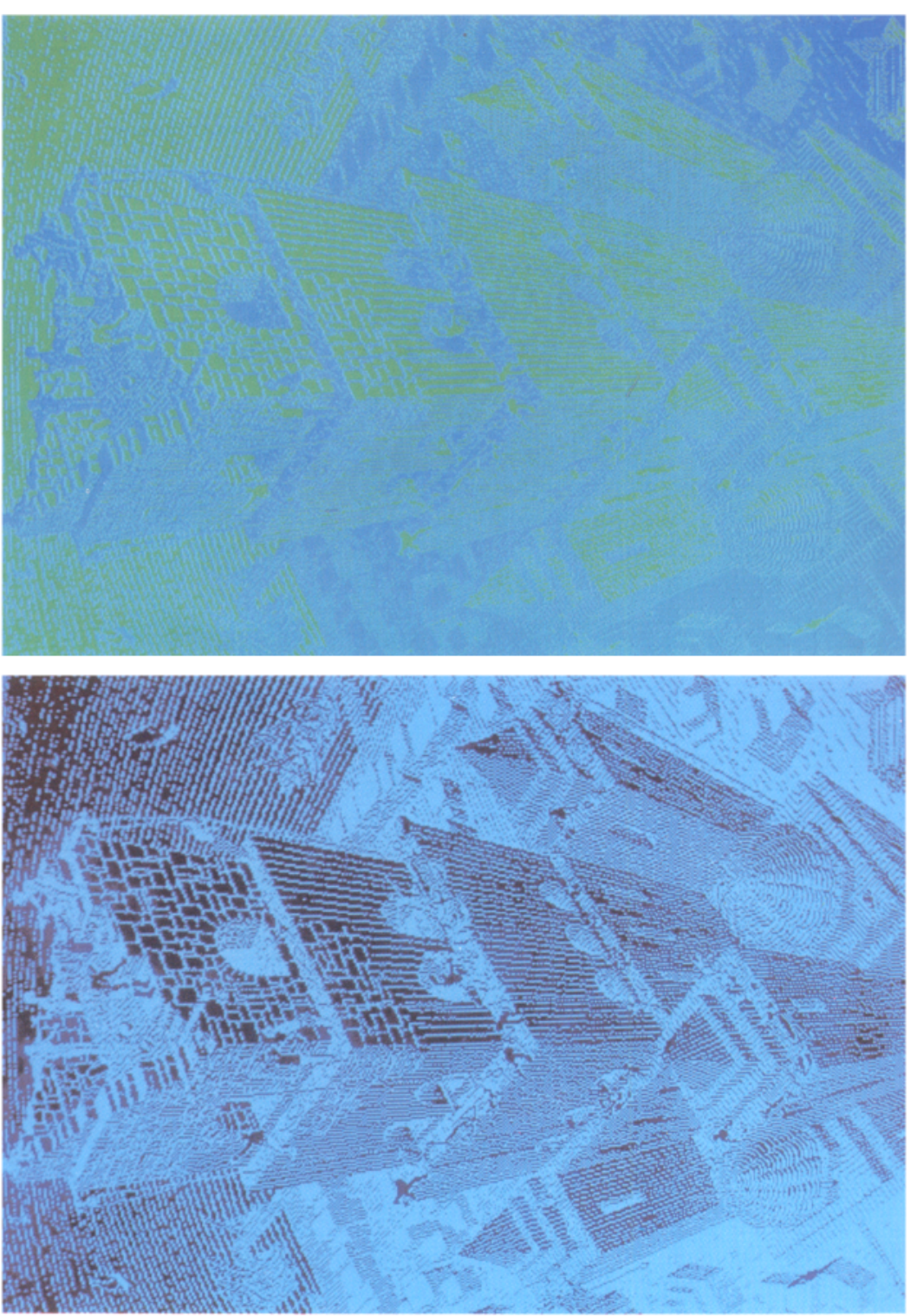

冚 苋

当

焉

密

해음

㟧.

จ :

응

窟

E응

害.

항

음

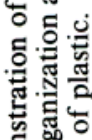

흥.

ᄃ

음

s

出是

ن

这焉

b

ब刍

政

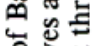

.

क

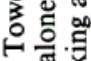

ㄱํㅇ응

늘

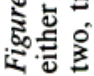




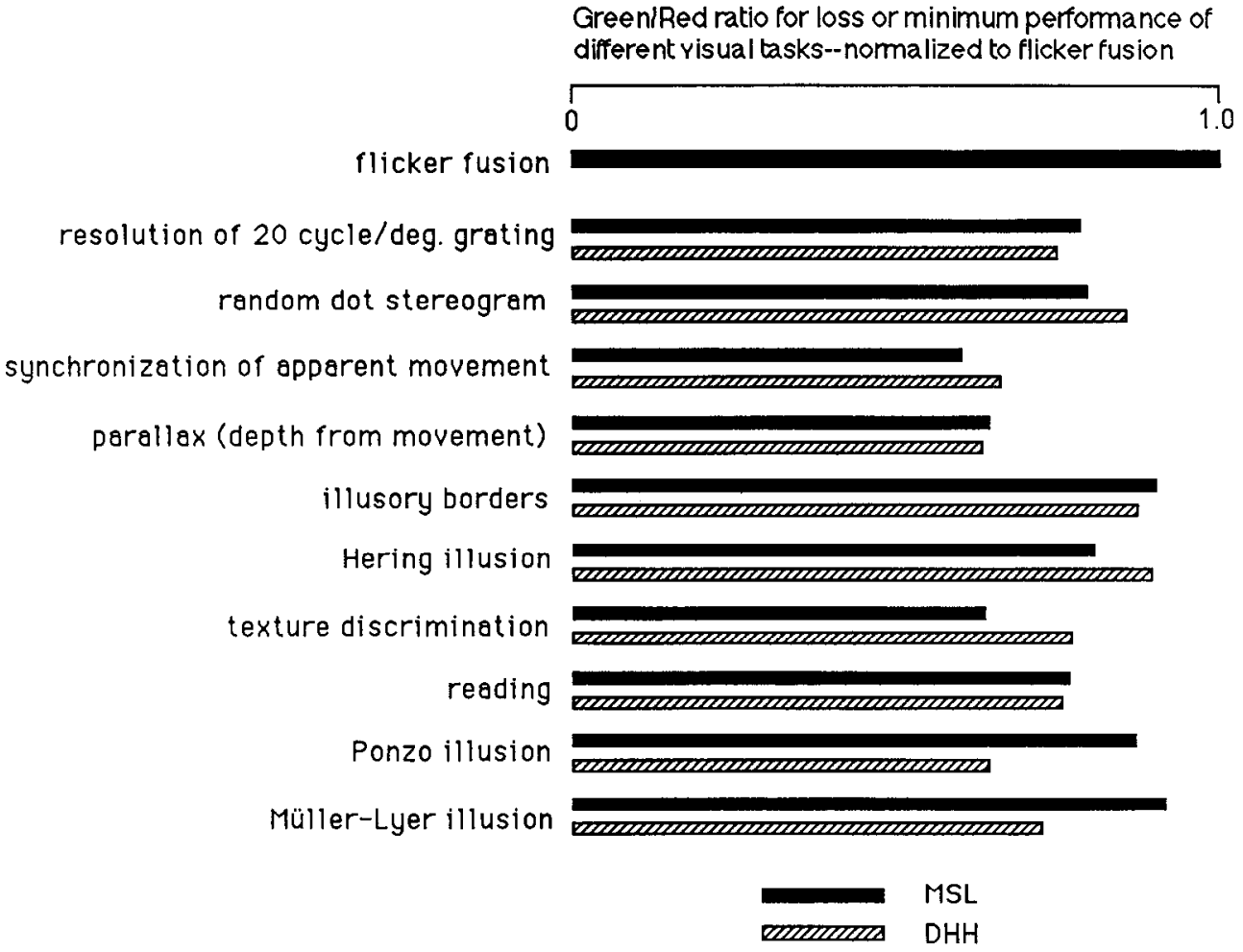

Figure 40. Red-to-green ratio at which different visual functions were lost or minimized. For both subjects, the red green ratio for flicker fusion was taken as 1.0 ; the rest were normalized to that.
2 stimuli is one index for comparing the temporal limitations for different visual tasks. The results can then be compared with the known differences in temporal characteristics of the magno and parvo systems. We made displays to test the fastest rate at which we could detect alternations in color, lightness, orientation, form, apparent movement, and disparity. The display (Fig. 41) subtended $2^{\circ}-5^{\circ}$, and we fixated on the center of each. (Maximum rates generally were higher more peripherally in the visual field, but we did not investigate this.) For shape, we estimated the highest rate at which we could see a circle and a square (of equal area) when the display alternated between the two. For orientation discrimination, a set of diagonal lines went from one orientation to the orthogonal orientation. For color discrimination, we alternated a red and green spot, set to equiluminance by flicker photometry, and determined the highest rate at which we could tell that the 2 colors were red and green. In Table 6, this is listed as "color alternation," and gave rates somewhat lower than those at which all flicker vanished, which we term "color flicker." Luminance flicker was the rate at which a black-white alternation fused. For stereopsis we fused two $1^{\circ} \times 1^{\circ}$ white squares in reference frames, one for each eye, and shifted the disparity of the squares, producing a sensation of movement in depth. We tested the ability to follow apparent movement of a simple figure as shown in Figure 41. We tested the speed of the illusory contour-generating system by shifting the position of an illusory contour as shown. In this test we would, of course, only be able to follow at a rate limited by whichever process is the slower, generating an illusory border or following apparent movement, but we were able to follow an illusory border at all rates at which we could see apparent movement. We have included in this comparison Krauskopf's (1957) datum on the rate of vibratory movement effective in overcoming fading of a stabilized image.
The results in Table 6 indicate that the system or systems underlying movement, stereopsis, luminance contrast, and the perception or integration of illusory contours can all follow more rapid events than the systems subserving color, form, or the prevention of image fading by eye movements. This is consistent with the idea that the former are all functions of the magno system and the latter functions of the slower parvo system.

Possible isolation of magnocellular pathway functions using 15 $\mathrm{Hz}$ counterphase flickering stimuli

To explore further the psychophysical consequences of the temporal differences between the magno and parvo systems, we asked if we could use these temporal differences to devise stimuli

\begin{tabular}{lcc}
\hline Table 6. Maximum alternation frequency for different visual tasks \\
\\
$\begin{array}{lcc}\text { Maximum rate } \\
\text { (cycles/sec) }\end{array}$ \\
\cline { 2 - 3 } Test & M.S.L. & D.H.H. \\
\hline Shape discrimination & 5 & 4 \\
Orientation discrimination & 5 & 5 \\
Color alternation & 5 & 5 \\
Color flicker & 7 & 7 \\
Luminance flicker & 30 & 30 \\
Apparent movement & 15 & 15 \\
Illusory border (apparent movement) & 15 & 10 \\
Stereopsis & 15 & 15 \\
Vibration rate to restore visibility of a & & \\
$\quad$ stabilized image & &
\end{tabular}

${ }^{a}$ From Krauskopf (1957). 

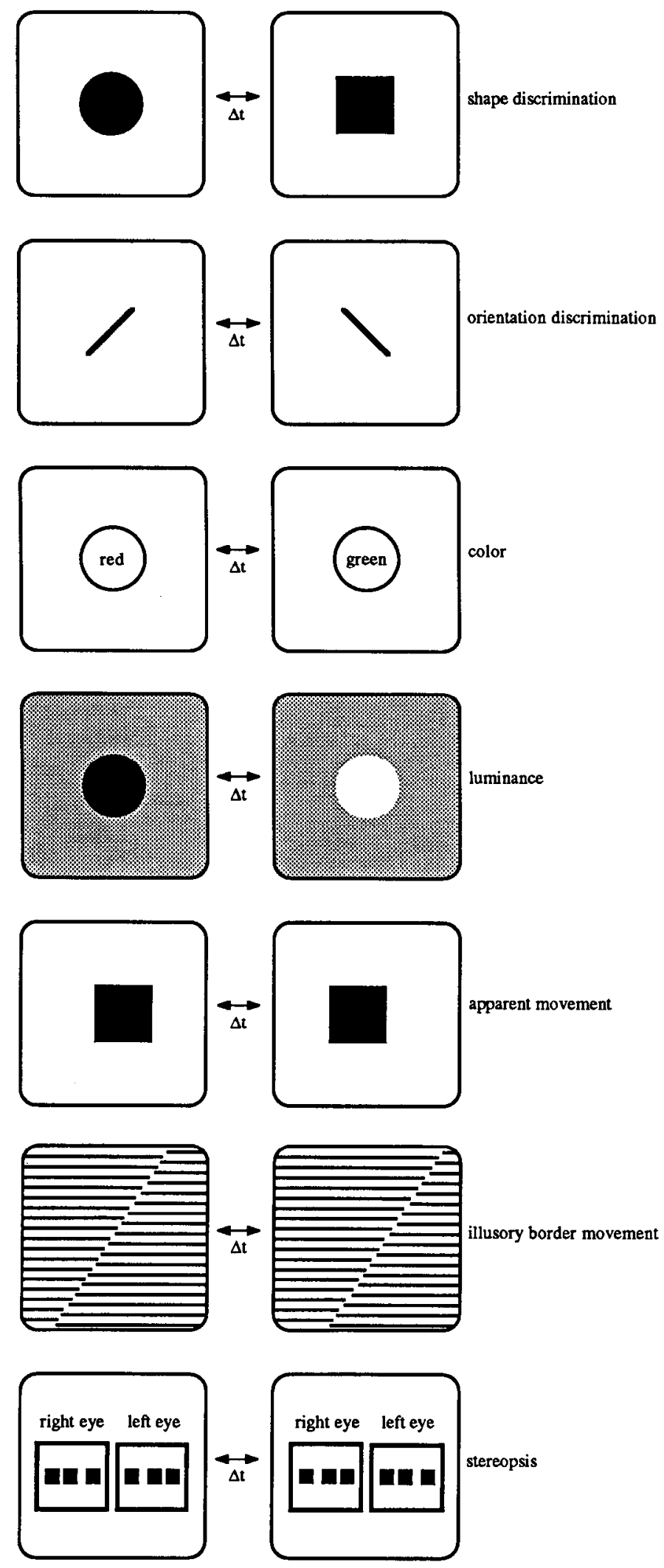

Figure 41. Stimuli used to measure maximum alternation rates listed in Table 6 for various visual tasks. For each of the 7 examples, the display alternated between the picture on the right and the one on the left. The time each picture was on could be varied, so that the alternation rate varied. In each case, we measured the maximum rate at which the 2 figures could each be distinguished. For the top pair, we measured the maximum rate at which the subject could still tell that a circle was that could be seen only by the faster magno system. We have already discussed the fact that, for a single spot alternating between 2 colors (Fig. $42 a$ ) at alternation rates below $15 \mathrm{~Hz}$, both color and luminance alternations can be seen; between 15 and $40 \mathrm{~Hz}$, colors fuse but luminance alternations still produce a sensation of flicker; above $40-60 \mathrm{~Hz}$, both color and luminance fuse. Physiologically, the magno system has a faster temporal frequency cutoff than the parvo system, so at least the magno system should be responsive at the highest detectable alternation rates; but we cannot predict from the physiology whether only one or both of the other 2 systems are unresponsive above 15 $\mathrm{Hz}$. The fact that the perceived hue is affected above $15 \mathrm{~Hz}$ suggests that at least the color-opponent cells in the blobs cannot follow alternations faster than $15 \mathrm{~Hz}$.

To see whether the interblob system might also be unresponsive above $15 \mathrm{~Hz}$, we used, instead of a single-colored square, a square that was half red and half green (Fig. $42 b$ ). We would predict that, with this stimulus, at nonequiluminance the hue of the squares would be determined by the blob system, and the vertical border would be seen by both the parvo-interblob system and the magno system. At equiluminance, however, the hue should still be seen by the blob system, but the border should be seen only by the parvo-interblob system and not by the magno system. The temporal limitations of the parvo-interblob system should therefore determine the rate at which one can still see the vertical border at equiluminance.

We found that when the stimulus shown in Figure $42 b$, a square vertically divided into red on one side and green on the other, was viewed with the red and green alternating at $15 \mathrm{~Hz}$, the vertical border completely disappeared at equiluminance. At this rate we could not see any color alternation: the red and green fused and appeared yellow or brown, though at nonequiluminance we could still see a luminance flicker. All 5 of the observers we tested found that they could see the border disappear entirely, though the ratio of red to green was quite critical, and it was necessary for the observer to fixate, because the border reappeared with eye movements. At nonequiluminance, the vertical border was always clearly visible, separating 2 apparently identical flickering yellow areas.

Since the border disappears at equiluminance, the visibility of the border at nonequiluminance must be due to activity in the magno system, and this raises the possibility that such rapidly alternating borders, like very low luminance-contrast stimuli, are another way to distinguish between magno and parvo

alternating with a square; at faster rates, they fused into a shape with rounded sides and square corners. Similarly, for orientation, the subject indicated when he could see 2 lines with different orientations and when they fused to form an $\times$. We took 2 rates for color alternation: the maximum rate at which the subject could tell that the 2 alternating colors were red and green was called "color alternation," and the maximum rate at which the subject still saw some flicker (even at equiluminance) we called "color flicker." Luminance flicker was the maximum rate at which the subject could still see flicker for alternating black and white. For apparent movement we measured the fastest rate at which the square appeared to move laterally; at faster rates it looked like a stationary square with blurred sides. For the illusory border we took the maximum rate at which the subject could see a coherent movement of the entire illusory contour; at higher rates the ends of the lines seemed to jiggle independently and to blur. For stereopsis we measured the fastest rate at which the central square seemed to move in depth; at faster rates the sides blurred, and the square appeared to lie in the plane of the screen. 
functions. We have tentatively attributed many complex visual functions solely to the magno system because they are lost or diminished at equiluminance, but retained at very low luminance contrast; these functions include stereopsis, movement detection, depth from perspective, parallax and other nonstereoscopic cues, generation of illusory borders, and figure/ground discrimination. With patterns consisting of only very low luminance-contrast isochromatic borders, surprisingly few specific visual functions (except of course color perception) are missing. The counterintuitive conclusion from these results is that the only functions of the massive parvocellular division of the primate visual system are to increase visual resolution by a factor of 2 or 3 over that possible with the more primitive magnocellular system, and to be able to use color to help define borders. We therefore welcomed the possibility of another potential way to isolate psychophysically the visual abilities of the magno system.

Luminance-contrast borders alternating at $15 \mathrm{~Hz}$ appeared very clear, and they had the same sharp, vanishingly thin quality seen in illusory borders and in borders defined solely by movement or by stereopsis. We could easily discriminate shapes defined by these borders, and could read text. Visual acuity, as measured by the ability to detect a square-wave grating, was lower by a factor of 2.5 than with stationary (nonalternating) gratings or gratings alternating slower than at $7 \mathrm{~Hz}(12$ cycles/ deg vs 30 ); a stationary equiluminant color-contrast grating could be resolved up to $18 \mathrm{cycles} / \mathrm{deg}$.

Using $15 \mathrm{~Hz}$ counterphase alternating luminance-contrast patterns, we could detect apparent movement, stereopsis (even when the input to the 2 eyes was of opposite phase), illusory contours (Fig. 42c), size distortion due to perspective in the Ponzo illusion (Fig. 42d), and the illusory offset of the Poggendorff illusion (Fig. 42e). The visual functions retained under rapid alternation are the same as those retained at very low luminance contrast and lost at equiluminance. Thus these results support the idea that rapidly alternating patterns are seen only by the magno system.

\section{Loss of magnocellular functions with voluntary fixation}

We think that one way to see what functions the parvo system is capable of is to eliminate the magno system, using equiluminant patterns; but this is a tricky technique and difficult to produce without a color monitor. Our results with fading suggest that the magno system habituates quickly during voluntary fixation, and, except at equiluminance, the parvo system does not. We therefore thought that this might be a simple way to demonstrate the effects of inactivity in the magno system.

We found that what we did in order to achieve the fading of low-contrast images felt like staring off into space or not paying attention to visual input. It may be that the peculiar and flat quality of vision at such moments is due to adaptation of the magno system. We then looked to see if functions we had previously attributed to the magno system also disappeared during fixation, and indeed they did. During fixation on a single dot in a random-dot stereogram, the depth sensation would transiently disappear, especially if we picked a point of fixation away from the center of the stereogram. We found that perspective, illusions, shape from texture and shading, and illusory borders all tended to vanish during fixation, even though, just as with equiluminant patterns, the images themselves were still quite clear. We suggest that the reader try fixating, especially eccentrically, on the images in Figures 27, 28, 31, 36, and 37-39, and compare
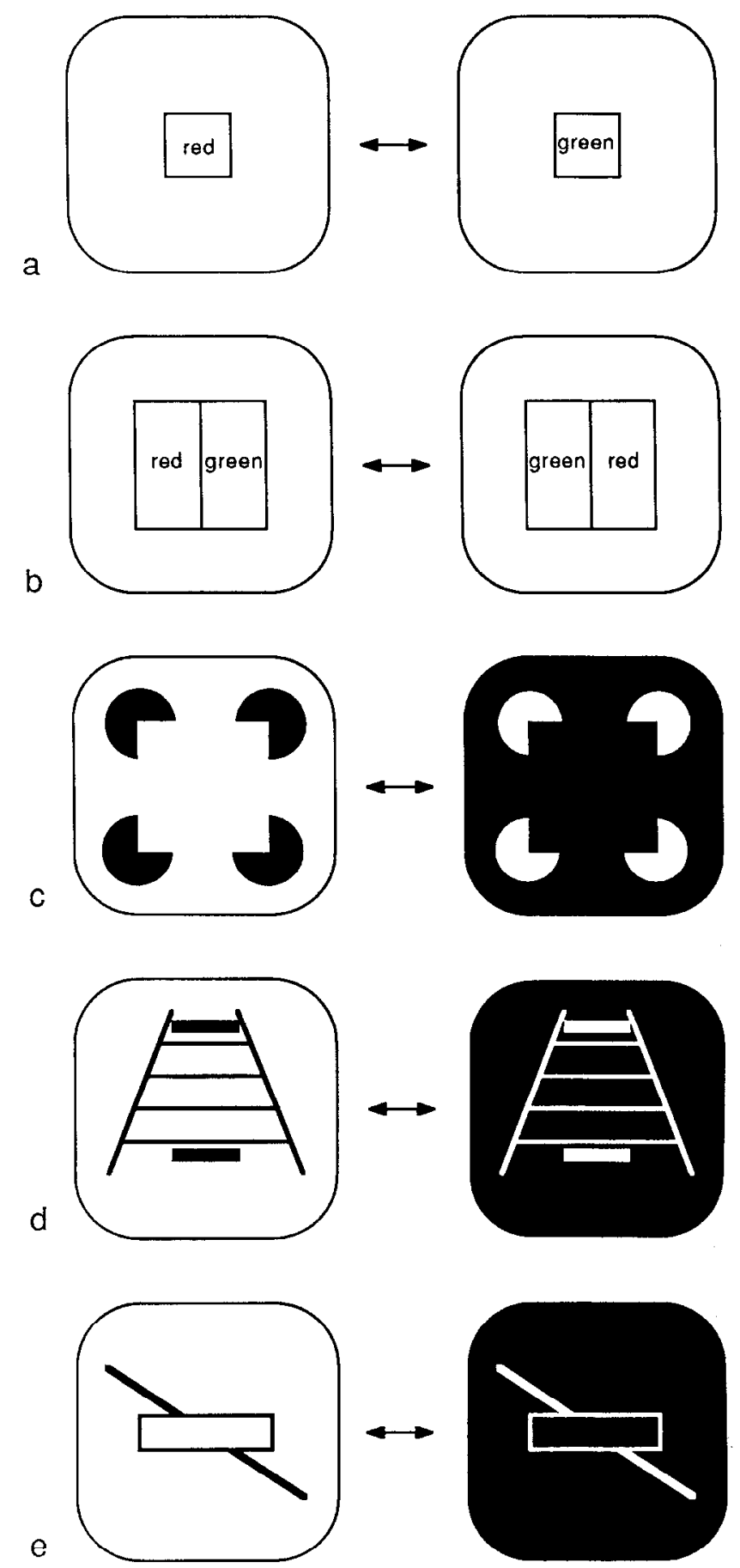

Figure 42. a, A single square alternates between red and green at different frequencies. Between 15 and $40 \mathrm{~Hz}$ the colors fuse to form yellow, but a luminance flicker persists. $b-e$, Counterphase flickering stimuli that we suspect may be seen by the magno system but not by the parvo system. All of the borders and images disappeared at equiluminance, but, at nonequiluminance, all of the illusions were still intact and one could still see the illusory contours.

your perception during fixation (marking a small fixation point helps) to that with ordinary eye movements.

\section{Do blue cones contribute to the luminance (magno) system?}

Throughout this paper we have ignored the blue cones, and in discussing luminance contrast we confined our consideration to 
the influence of the red and green cones, because the contribution from the bluc concs to the luminance system is small at most, and there is some controversy over whether the blue cones contribute at all. Originally, reports that the blue cones do not contribute to the luminosity system were based on 2 different psychophysical measurements of luminosity function: flicker photometry (Eisner and MacLeod, 1980) and the "minimally distinct border" psychophysical criterion (Tansley and Boynton, 1978). But recently studies by the same laboratories, using more sensitive tests, have revealed a blue cone contribution to both flicker photometry at high frequencies $(>30 \mathrm{~Hz})$ and border distinctness (Boynton et al., 1985; Kaiser and Boynton, 1985; Stockman and MacLeod, 1986). Furthermore, Grinberg and Williams (1985) compared stereo acuity for 440 and $480 \mathrm{~nm}$ stimuli and reported that the blue system contributes to stereopsis. Physiological reports have shown similar disagreement Macaque A-type retinal ganglion cells and magnocellular geniculate cells have been reported to receive input from red and green cones only, and not from blue cones (Gouras, 1968; De Monasterio and Gouras, 1975), but other groups have reported that the magno system does receive a small input from the blue cones (Hubel and Wiesel, 1966; Derrington et al., 1984).

We attempted to test our stereopsis using information from the blue cones alone. We flooded a screen with a very bright $\left(60 \mathrm{~cd} / \mathrm{m}^{2}\right)$ diffuse yellow light $(580 \mathrm{~nm})$, and added to this a black-and-white random-dot stereopair projected through a 460 $\mathrm{nm}$ interference filter, producing a white image on a yellow background. We could easily see depth with this stimulus. We believe the stereogram selectively stimulated only the blue cones, because we could not see the blue light foveally: When we projected a 10 arc min dot with the same slide projector and 460 $\mathrm{nm}$ filter, with or without the yellow background, the dot disappeared when we looked directly at it, but was easily visible when we deviated our gaze by less than $14^{\circ}$. (Blue cones are absent in the fovea: König, 1894; Wald, 1967; Williams et al., 1981.) It is unlikely that rods could have been contributing to the result, given the brightness of the yellow light and the fact that the rod-blind region of the retina covers at least $1^{\circ}(\varnothing$ sterberg, 1935; Polyak, 1957; Williams et al., 1981).

Since both stereopsis and flicker above $30 \mathrm{cycles} / \mathrm{sec}$ can be mediated by the blue cones alone, we conclude that blue cones probably do contribute to the magno system, but that their contribution is likely to be very small, just like their numbers, relative to the red and green cones.

\section{Discussion}

We began this study because findings on the physiology and the specificity of the connections between areas 17 and 18 suggested a striking segregation of different visual functions, a segregation that could be traced back to the magno/parvo segregation in the lateral geniculate body. The marked differences in physiological properties between the 2 geniculate divisions led to specific predictions about the visual functions carried selectively by one or the other geniculate division. Depth and movement seem to be carried predominantly by the magnocellular division, and color perception by the blob system. Form perception is probably carried by both the magno system and the parvo-interblob system, but the 2 systems seem to carry different aspects of form information. The validity of this formulation is open to debate, but we have used it as a framework for organizing the psychophysical results. The segregation also seems clear enough to justify our asking how it squares with human psychophysics and with clinical observations.

It would be amazing if 2 centuries of psychophysics had not already answered many of these questions. Our Results section is therefore made up partly of a review of many old experiments, partly of our repetitions or variants of old work, and of attempts to fill in gaps. Because of the number and variety of experiments, we have not yet tested all of them rigorously, and for many, such as the disappearance of illusions and illusory contours at equiluminance or during fixation, we have only the subjective experiences of ourselves and a few other observers.

We have been gratified and surprised at how well the human psychophysics seems to fit the anatomy and physiology. Perhaps we can begin to be optimistic over the prospect of amassing a body of coherent neurobiological results on primates and humans that supports the idea of separate parallel paths for different visual functions. Much psychophysics had already pointed in that direction, and we have simply tried to gather it together-adding, where possible-in the context of the neurobiology. The picture is still far from complete; moreover, gaps in the evidence and a few anomalies certainly complicate our arguments. We are aware of the dangers of exaggerating the separateness of the systems, and of the temptations to do so when such a puzzle begins to make sense.

The evidence is strong that the visual system is divided into several subsystems operating independently and in parallel, sharing some properties but differing significantly in others. Though we can see evidence of this subdivision in our own visual perception, we nevertheless do not understand the functional significance of this organization; we do not know whether it is a salutary design principle or an accident of evolution. Subdividing a single map into functionally distinct modules would facilitate interactions, such as center-surround-opponency or lateral inhibition, among cells carrying the same type of information. Moreover, segregation of different functions could allow each subdivision to acquire properties that might be particularly suited to its specialization. For example, the fast temporal properties of the magno system should be particularly useful in movement analysis. The slower temporal properties of the parvo system might make it capable of discriminating stationary objects that the magno system might be blind to. The benefits of other differences, such as the magno system's insensitivity to color or the parvo system's lesser sensitivity to luminance contrast, are less obvious. There may be some use for stereopsis and movement detection's being color-blind, such as reducing ambiguities between an edge moving in one direction and an edge of the opposite contrast moving in the opposite direction.

If design principles were the only underlying explanation for segregation, one could wonder why the various tasks are not even more separate. One possible explanation is that some of the properties derive not from design, but from the constraints of evolution; they must have evolved, presumably by duplication, expansion, or alteration of preexisting structures. It is easy to imagine that evolution might produce a machine with each subsystem or parallel component sharing many design features and inputs with other subsystems; such a model would be consistent with the apparent redundancies in form perception and movement sensitivity.

From considerations of both development and evolution there are hints that the magno system is older and more primitive than the parvo system (Hubel et al., 1977; Kaas et al., 1978; 


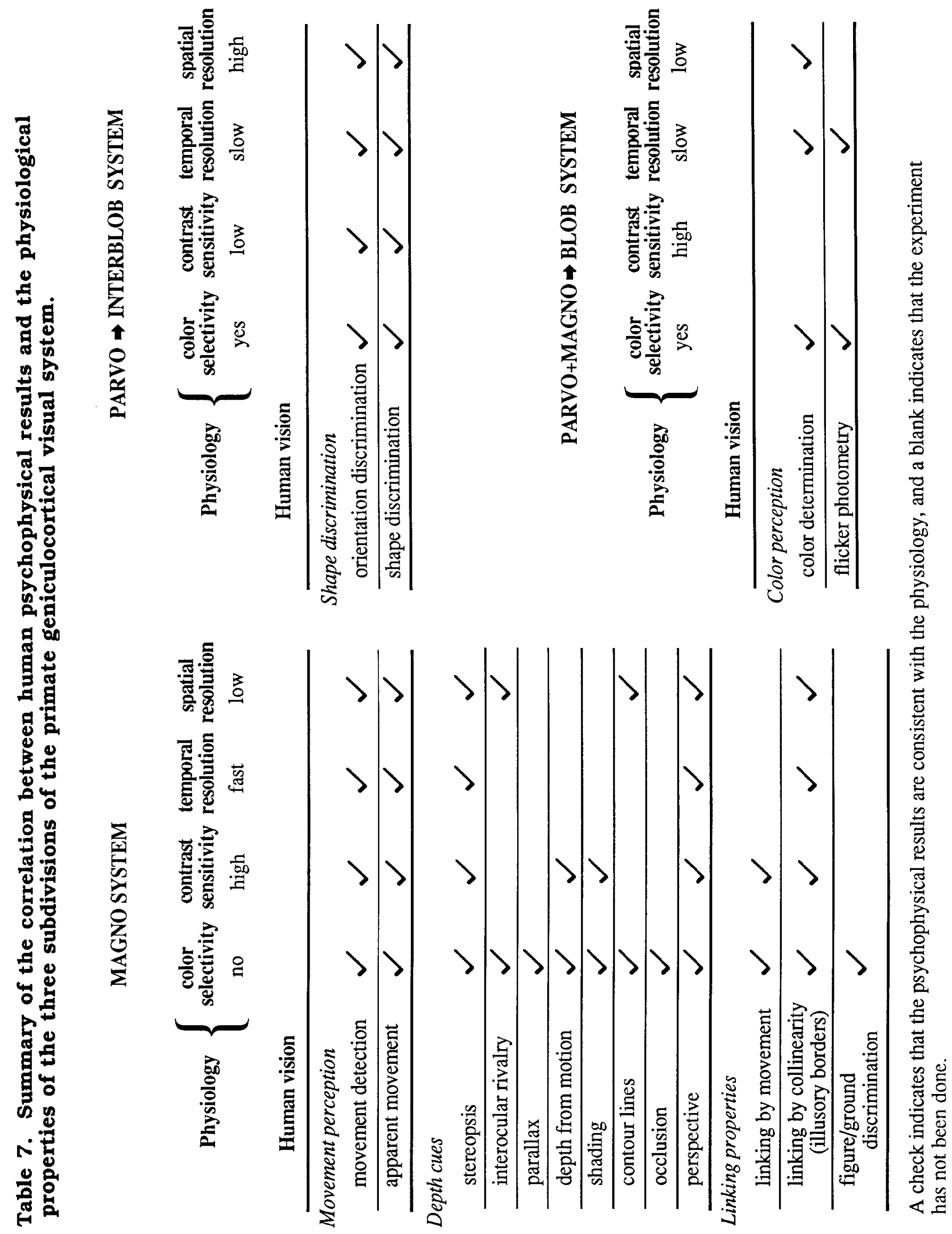


Guillery, 1979; Lennie, 1980; Sherman, 1985), so it may be that we should not ask what the magno system is specialized for, but rather what is added on by the parvo system. An animal whose parvo system, or its homolog, is not well developed may be fully capable of carrying out what we have been considering as the essential functions of vision, such as detecting, identifying, and following objects, especially moving ones, and determining their positions in space so that the animal can navigate through its environment. But such an animal might nevertheless see the world very differently from the way we see it: it should see only the outlines of objects, with all homogeneous surfaces appearing the same, not differing in color or lightness, and it should be able to distinguish only moving objects well.

We have suggested that both the magno and the parvo system can convey information about form, but that they may be involved in different types of form discrimination. Having 2 systems analyzing the same information might allow them to perform very different, perhaps mutually exclusive, types of calculations on that information. The magno system seems to be wired up in such a way that it tends to integrate information from different parts of the visual field; it seems to be able to correlate and link spatially separate stimuli. The parvo-interblob system, by contrast, seems to be more spatially restrictive. Perhaps because linking functions are already taken care of by the magno system, the parvo system can concentrate on a finer analysis of the same information. For example, operations such as end-stopping might interfere with linking calculations, but would be very useful in conveying fine, detailed, complicated shape. By adding the parvo-interblob and blob systems we acquire a 2-fold higher resolution and the ability to distinguish surfaces as particular colors, and possibly as shades of gray as well. An interesting possibility suggested by the results on image fading is that the parvo system also confers the ability to see well, or perhaps in finer detail, the static components of our environment. Lu and Fender (1972) have suggested that stereopsis is color-blind because color vision evolved relatively recently; when the ability to use color information developed, the system or systems used to analyze stereopsis (and perhaps movement) may have already become segregated from the system used for high-resolution form analysis, so possibly the ability to use color information developed only in the high-resolution part of the form system. It is interesting, in this context, that the acrylamide-treated monkeys studied by Merigan and Eskin (1986) had significant histological damage to the entire parvocellular pathway, but showed only minor deficiencies in visual performance.

It seems highly likely that other sensory systems will show analogous parallel paths for handling different aspects of information. For audition, it is clear from clinical observations in man that comprehension of spoken language has its own special apparatus. For somatic sensation one might be tempted to point to the paths subserving touch, pain, and position sense as an example of processing of sensory information along 3 parallel, separate channels, but the analogy here is weakened by the fact that the receptors for the 3 channels are different. In the case of position versus touch, even the receptor locations are different, so the surfaces that are mapped are similar only in the loosest sense. This is not to say that, at some central level, different aspects of a single modality such as touch will not turn out to be handled separately.

Because of the number of results presented here, we have summarized them in Table 7.
As Rushton had hoped, we find that the psychophysics indeed leads us to new anatomical and physiological questions-at the risk of further thickening the forest.

\section{References}

Adrian, E. D. (1928) The Basis of Sensations, Norton, New York.

Allman, J., F. Miezin, and E. L. McGuinness (1985a) Direction- and velocity-specific responses from beyond the classical receptive field in the middle temporal visual area (MT). Perception 14: 105-126.

Allman, J., F. Miezin, and E. L. McGuinness (1985b) Stimulus specific responses from beyond the classical receptive field: Neurophysiological mechanisms for local-global comparisons in visual neurons. Annu. Rev. Neurosci. 8: 407-430.

Alpern, M. (1972) In Handbook of Sensory Physiology, vol. VII/4, chapt. 12 (from the series Visual Psychophysics), D. James and L. M. Hurvich, eds., pp. 303-330, Springer-Verlag, Berlin, Heidelberg, New York.

Anstis, S. M. (1980) The perception of apparent movement. Phil. Trans. R. Soc. Lond. [Biol.] 290: 153-168.

Anstis, S. M., and P. C. Cavanagh (1983) A minimum motion tcchnique for judging equiluminance. In Colour Vision: Physiology and Psychophysics, J. Mollon and R. T. Sharpe, eds., pp. 155-166, Academic, New York.

Anstis, S. M., and G. Mather (1985) Effects of luminance and contrast on direction of ambiguous apparent motion. Perception 14: 167-179.

Barlow, H. B. (1972) Visual pattern analysis in machines and animals. Science 177: 567-575.

Barlow, H. B. (1981) Critical limiting factors in the design of the eye and visual cortex. (The Ferrier Lecture, 1980.) Proc. R. Soc. Lond. [Biol.] 212: 1-34.

Barlow, H. B., C. Blakemore, and J. D. Pettigrew (1967) The neural mechanism of binocular depth discrimination. J. Physiol. (Lond.) 193: 327-342.

Berliner, A., and S. Berliner (1948) The distortion of straight and curved lines in geometrical fields. Am. J. Psychol. 61: 153-166.

Bernoulli, J. (1705) On his tombstone in the Münster, Basel, Switzerland.

Bishop, P. O., G. H. Henry, and C. J. Smith (1971) Binocular interaction fields of single units in the cat striate cortex. J. Physiol. (Lond.) 216: 39-68.

Blakemore, C., and F. Vital-Durand (1981) Distribution of X-and $\mathrm{Y}$-cells in the monkey's lateral geniculate nucleus. J. Physiol. (Lond.) 320: 17P-18P

Blakemore, C., R. H. S. Carpenter, and M. A. Georgeson (1970) Lateral inhibition between orientation detectors in the human visual system Nature 228: 37-39.

Blasdel, G. G., and D. Fitzpatrick (1984) Physiological organization of layer 4 in macaque striate cortex. J. Neurosci. 4: 880-895.

Bouma, H., and J. J. Andriessen (1970) Induced changes in the perceived orientation of line segments. Vision Res. 10: 333-349.

Boycott, B. B., and H. Wässle (1974) The morphological types of ganglion cells of the domestic cat retina. J. Physiol. (Lond.) 240:397419.

Boynton, R. M., R. T. Eskew, Jr., and C. X. Olson (1985) Blue cones contribute to border distinctness. Vision Res. 25: 1349-1352.

Braddick, O. (1974) A short range process in apparent motion. Vision Res. 14: 519-528.

Brussell, E. M., S. R. Stober, and D. M. Bodinger (1977) Sensory information and subjective contour. Am. J. Psychol. 90: 145-156.

Bullier, J., and G. H. Henry (1980) Ordinal position and afferent input of neurons in monkey striate cortex. J. Comp. Neurol. 192: 913-935.

Campbell, F. W., and L. Maffei (1981) The influence of spatial frequency and contrast on the perception of moving patterns. Vision Res. 21: 713-721.

Cavanagh, P., and O. E. Favreau (1985) Color and luminance share a common motion pathway. Vision Res. 25: 1595-1601.

Cavanagh, P., C. W. Tyler, and O. E. Favreau (1984) Perceived velocity of moving chromatic gratings. J. Opt. Soc. Am. (A. Op. Image Sci. Ser.) 1: 893-899.

Cavanagh, P., J. Boeglin, and O. E. Favreau (1985) Perception of motion in equiluminous kinematograms. Perception 14: 151-162.

Cavonius, C. R., and A. W. Schumacher (1966) Human visual acuity measured with colored test objects. Science 152: 1276-1277.

Chang, J. J., and B. Julesz (1984) Cooperative phenomena in apparent 
movement perception of random-dot cinematograms. Vision Res. 24: $1781-1788$.

Chaudhuri, A., and D. A. Glaser (1986) A new anisotropy in apparent motion. (ARVO Abstr. 32.) Invest. Ophthalmol. Vis. Sci. (Suppl.) 27: 345.

Chevreul, M. E. (1839) De la Loi du Contraste Simultané des Coleurs, Pitois-Levrault, Paris.

Clarke, P. G. H., I. M. L. Donaldson, and D. Whitteridge (1976) Binocular visual mechanisms in cortical areas I and II of the sheep. J. Physiol. (Lond.) 256: 509-526.

Comerford, J. P. (1974) Stereopsis with chromatic contours. Vision Res. 14: 975-982.

Cornsweet, T. N. (1966) Stabilized image techniques. In Recent Developments in Vision Research, A. Whitcomb, ed., pp. 171-184, National Academy of Sciences, Washington, DC.

Cuxeter, H. S. M. (1969) Introduction to Geometry, Wiley, New York.

Curcio, C. A., and J. K. Harting (1978) Organization of pulvinar afferents to area 18 in the squirrel monkey: Evidence for stripes. Brain Res. 143: 155-161.

Damasio, A., T. Yamada, H. Damasio, J. Corbett, and J. McKee (1980) Central achromatopsia: Behavioral, anatomic, and physiologic aspects. Neurology 30:1064-1071.

Daw, N. W., and A. L. Pearlman (1970) Opponent color cells in the cat lateral geniculate nucleus. Science 167: 84-86.

Delboeuf, J. R. L. (1893) Une nouvelle illusion d'optique. Bull. Acad. R. Belg. 24: S545.

De Monasterio, F. M., and P. Gouras (1975) Functional properties of ganglion cells of the rhesus monkey retina. J. Physiol. (Lond.) 251: 167-195.

Derrington, A. M., and P. Lennie (1984) Spatial and temporal contrast sensitivities of neurones in lateral geniculate nucleus of macaque. $\mathbf{J}$. Physiol. (Lond.) 357: 219-240.

Derrington, A. M., J. Krauskopf, and P. Lennie (1984) Chromatic mechanisms in lateral geniculate nucleus of macaque. J. Physiol. (Lond.) 357: 241-265.

De Valois, K. K., and E. Switkes (1983) Simultaneous masking interactions between chromatic and luminance gratings. J. Opt. Soc. Am. 73: 11-18.

De Valois, R. L., E. Abramov, and G. H. Jacobs (1966) Analysis of response patterns of LGN cells. J. Opt. Soc. Am. 56: 966-977.

De Valois, R. L., D. M. Snodderly, Jr., E. W. Yund, and N. K. Hepler (1977) Responses of macaque lateral geniculate cells to luminance and color figures. Sens. Processes 1: 244-259.

de Weert, C. M. M. (1979) Colour contours and stereopsis. Vision Res. 19:555-564.

de Weert, C. M. M., and K. J. Sadza (1983) New data concerning the contribution of colour differences to stereopsis. In Colour Vision: Physiology and Psychophysics, J. D. Mollon and L. T. Sharpe, eds., pp. 553-562, Academic, New York

DeYoe, E. A., and D. C. Van Essen (1985) Segregation of efferent connections and receptive field properties in visual area V2 of the macaque. Nature 317: 58-60.

Ditchburn, R. W., and J. A. Foley-Fisher (1967) Assembled data in eye movements. Optica Acta 14: 113-118.

Ditchburn, R. W., and B. L. Ginsborg (1952) Vision with a stabilized retinal image. Nature 170: 36-37.

Ditchburn, R. W., D. H. Fender, and S. Mayne (1959) Vision with controlled movements of the retinal image. J. Physiol. (Lond.) 145: 98-107.

Dow, B. (1974) Functional classes of cells and their laminar distribution in monkey visual cortex. J. Neurophysiol. 37: 927-946.

Dreher, B., Y. Fukada, and R. W. Rodieck (1976) Identification, classification and anatomical segregation of cells with $X$-like and $Y$-like properties in the lateral geniculate nucleus of old-world primates. $J$. Physiol. (Lond.) 258: 433-452.

Dubner, R., and S. M. Zeki (1971) Response properties and receptive fields of cells in an anatomically defined region of the superior temporal sulcus. Brain Res. 35: 528-531.

Eisner, A., and I. A. MacLeod (1980) Blue-sensitive cones do not contribute to luminance. J. Opt. Soc. Am. 70: 121-123.

Enroth-Cugell, C., and J. G. Robson (1966) The contrast sensitivity of retinal ganglion cells of the cat. J. Physiol. (Lond.) 187: 517-552.

Eskin, T. A., and W. H. Merigan (1986) Selective acrylamide-induced degeneration of color opponent ganglion cells in macaques. Brain Res. 378: 379-384.
Favreau, O., V. F. Emerson, and M. C. Corballis (1972) Motion perception; A color-contingent aftereffect. Science 176: 78-79.

Fitzpatrick, D., K. Itoh, and I. T. Diamond (1983) The laminar organization of the lateral geniculate body and the striate cortex in the squirrel monkey (Saimiri sciureus). J. Neurosci. 3: 673-702.

Fitzpatrick, D., J. S. Lund, and G. G. Blasdel (1985) Intrinsic connections of macaque striate cortex: Afferent and efferent connections of lamina 4C. J. Neurosci. 5: 3329-3349.

Gengerelli, J. A. (1948) Apparent movement in relation to homonymous and heteronymous stimulation of the cerebral hemispheres. $J$. Exp. Psychol. 38: 592-599.

Gibson, J. J. (1950) Perception of the Visual World, Riverside, Cambridge, MA

Gillam, B. (1980) Geometrical illusions. Sci. Am. 242: 102-111.

Gouras, P. (1968) Identification of cone mechanisms in monkey ganglion cells. J. Physiol. (Lond.) 199: 533-547.

Gouras, P. (1969) Antidromic responses of orthodromically identified ganglion cells in monkey retina. J. Physiol. (Lond.) 204: 407-419.

Gouras, P., and J. Krüger (1979) Kesponses of cells in foveal visual cortex of the monkey to pure color contrast. J. Neurophysiol. 42: 850860.

Gouras, P., and E. Zrenner (1979) Enhancement of luminance flicker by color-opponent mechanisms. Science 205: 587-589.

Granger, E. M., and I. C. Heurtley (1973) Visual chromaticity-modulation transfer function. J. Opt. Soc. Am. 63: 1173-1174.

Green, M. (1986) What determines correspondence strength in apparent motion? Vision Res. 26: 599-607.

Gregory, R. L. (1963) Distortion of visual space as inappropriate constancy scaling. Nature 199: 678-680.

Gregory, R. L. (1977) Vision with equiluminant colour contrast: 1. A projection technique and observations. Perception 6: 113-119.

Gregory, R. L., and J. P. Harris (1984) Real and apparent movement nulled. Nature 307: 729-730.

Grinberg, D. L., and D. R. Williams (1985) Stereopsis with chromatic signals from the blue-sensitive mechanism. Vision Res. 25: 531-537.

Guillery, R. W. (1979) A speculative essay on geniculate lamination and its development. In Development and Chemical Specificity of Neurons, Progress in Brain Research, vol. 51, M. Cuenod, G. W. Kreutzberg, and F. E. Bloom, eds., pp. 403-418, Elsevier/NorthHolland, Amsterdam.

Harris, C. S., and B. Barkow (1969) Color/white grids produce weaker orientation-specific color aftereffects than do color/black grids. Psychonom. Sci. (Abstr.) 17: 123.

Hawken, M. J., and A. J. Parker (1984) Contrast sensitivity and orientation selectivity in lamina IV of the striate cortex of old world monkeys. Exp. Brain Res. 54: 367-372.

Helmholtz, H. von (1910) Handbuch der Physiologischen Optik, Verlag von Leopold Voss, Hamburg und Leipzig.

Hepler, N. K. (1968) Color: A motion-contingent aftereffect. Science 162: 376-377.

Hering, E. (1861) Beitrage zur Physiologie, vol. 1, pp. 64-80, Leipzig. Hering, E. (1893) Ueber den Einfluss der Macula Lutea auf spectrale Farbengleichungen. Pfluegers Arch. 54: 277-312.

Hicks, T. P., B. B. Lee, and T. R. Vidyasagar (1983) The responses of cells in macaque lateral geniculate nucleus to sinusoidal gratings. J. Physiol. (Lond.) 337: 183-200.

Hubel, D. H., and M. S. Livingstone (1985) Complex-unoriented cells in a subregion of primate area 18 . Nature $315: 325-327$

Hubel, D. H., and M. S. Livingstone (1987) Segregation of form, color, and stereopsis in primate area 18. J. Neurosci. 7: 3378-3415.

Hubel, D. H., and T. N. Wiesel (1962) Receptive fields, binocular interaction and functional architecture in the cat's visual cortex. $J$. Physiol. (Lond.) 160: 106-154.

Hubel, D. H., and T. N. Wiesel (1965) Binocular interaction in striate cortex of kittens reared with artificial squint. J. Neurophysiol. 28: $1029-1040$

Hubel, D. H., and T. N. Wiesel (1966) Spatial and chromatic interactions in the lateral geniculate body of the rhesus monkey. J. Neurophysiol. 29: 1115-1156.

Hubel, D. H., and T. N. Wiesel (1968) Receptive fields and functional architecture of monkey striate cortex. J. Physiol. (Lond.) 195:215243.

Hubel, D. H., and T. N. Wiesel (1970) Cells sensitive to binocular depth in area 18 of the macaque monkey cortex. Nature 225: 41-42.

Hubel, D. H., and T. N. Wiesel (1972) Laminar and columnar dis- 
tribution of geniculo-cortical fibers in the macaque monkey. J. Comp. Neurol. 146: 421-450.

Hubel, D. H., and T. N. Wiesel (1974) Sequence regularity and geometry of orientation columns in the monkey striate cortex. J. Comp. Neurol. 158: 267-294.

Hubel, D. H., T. N. Wiesel, and S. LeVay (1977) Plasticity of ocular dominance columns in monkey striate cortex. Phil. Trans. R. Soc. Lond. [Biol.] 278: 377-409.

Hurvich, L. M. (1981) Color Vision, Sinauer, Sunderland, MA.

Ives, H. E. (1923) A chart of the flicker photometer. J. Opt. Soc. Am. Rev. Sci. Instrum. 7: 363-365.

Julesz, B. (1960) Binocular depth perception of computer-generated patterns. Bell Syst. Technol. J. 39: 1125-1161.

Julesz, B. (1971) Foundations of Cyclopean Perception, U. Chicago P., Chicago.

Julesz, B., and B. White (1969) Short term visual memory and the Pulfrich phenomenon. Nature 222: 639-641.

Kaas, J. H., M. F. Huerta, J. T. Weber, and J. K. Harting (1978) Palterns of retinal terminations and laminar organization of the lateral geniculate nucleus of primates. J. Comp. Neurol. 182: 517-554.

Kaiser, P. K., and R. M. Boynton (1985) Role of the blue mechanism in wavelength discrimination. Vision Res. 25: 523-529.

Kanizsa, G. (1955) Margini quasi-percettivi in campi con stimulazioni omogonea. Riv. Psicologia 49: 7-30.

Kanizsa, G. (1974) Contours without gradients or cognitive contours? It. J. Psychol. 1: 93-112.

Kanizsa, G. (1979) Organization in Vision. Essays on Gestalt Perception, Praeger, New York.

Kaplan, E., and R. M. Shapley (1982) X and Y cells in the lateral geniculate nucleus of macaque monkeys. J. Physiol. (Lond.) 330:125143.

Kaplan, E., and R. M. Shapley (1986) The primate retina contains two types of ganglion cells, with high and low contrast sensitivity. Proc. Natl. Acad. Sci. USA 83: 2755-2757.

Keesey, U. (1972) Flicker and pattern detection: A comparison of thresholds. J. Opt. Soc. Am. 62: 446-448.

Kelly, D. H., and D. van Norren (1977) Time-based model of heterochromatic flicker. J. Opt. Soc. Am. 67: 1081-1091.

Koffka, K. (1935) Principles of Gestalt Psychology, Harcourt, Brace, New York, pp. 126-127.

Kolers, P. A., and J. R. Pomerantz (1971) Figural change in apparent motion. J. Exp. Psychol. 87: 99-108.

König, A. (1894) Uber den menschlichen Sehpurpur und seine Bedeutung für das Sehen, Vol. 30, pp. 577-598, S. B. Akad. Wissenshaft, Berlin

Krauskopf, J. (1957) Effect of retinal image motion on contrast thresholds for maintaincd vision. J. Opt. Soc. Am. 47: 740-744.

Krauskopf, J. (1961) Effect of retinal image stabilization on the appearance of heterochromatic targets. J. Opt. Soc. Am. 53: 741-744.

Krauskopf, J. (1967) Heterochromatic stabilized images: A classroom demonstration. Am. J. Psychol. 80: 632-637.

Krüger, J. (1977) Stimulus dependent colour specificity of monkey lateral geniculate neurones. Exp. Brain Res. 30:297-311.

Krüger, J. (1979) Responses to wavelength contrast in the afferent visual systems of the cat and the rhesus monkey. Vision Res. 19: $1351-1358$.

Kulikowski, J. J. (1971) Effects of eye-movements on the contrast sensitivity of spatio-temporal patterns. Vision Res. 11: 261-273.

Kulikowski, J. J., and D. J. Tolhurst (1973) Psychophysical evidence for sustained and transient detectors in human vision. J. Physiol. (Lond.) 232: 149-162.

Land, E. H. (1959) Color vision and the natural image. Part I. Proc. Natl. Acad. Sci. USA 45: 115-129.

Lehmann, A. (1904) Die irradiation als Ursache geometrische-optischer Tauschungen. Pfluegers Arch. 103: 84-106.

Lennie, P. (1971) Distortions of perceived orientation. Nature 233; $155-156$.

Lennie, P. (1980) Parallel visual pathways: A review. Vision Res. 20: $561-594$

Lettvin, J. Y. (1967) The colors of colored things. Q. Prog. Rep. Res. Lab. Electron. M.I.T. 87: 193-229.

Leventhal, A. G., R. W. Rodieck, and B. Dreher (1981) Retinal ganglion cell classes in the Old World monkey: Morphology and central projections. Science 213: 1139-1142.

Levi, D. M., and S. A. Klein (1986) Sampling in spatial vision. Nature $320: 360-362$
Liebmann, S. (1926) Uber das Verhalten farbiger Formen bei Helligkcitsglcichhcit von Figur und Grund. Psychol. Forsch. 9: 300-353.

Livingstone, M. S., and D. H. Hubel (1982) Thalamic inputs to cytochrome oxidase-rich regions in monkey visual cortex. Proc. Natl Acad. Sci. USA 79: 6098-6101.

Livingstone, M. S., and D. H. Hubel (1984a) Anatomy and physiology of a color system in the primate visual cortex. J. Neurosci. 4: 309356

Livingstone, M. S., and D. H. Hubel (1984b) Specificity of intrinsic connections in primate primary visual cortex. J. Neurosci. 4: 2830 2835 .

Livingstone, M. S., and D. H. Hubel (1987) Connections between layer 4B of area 17 and the thick cytochrome oxidase stripes of area 18 in the squirrel monkey. J. Neurosci. 7: 3371-3377.

$\mathrm{Lu}, \mathrm{C}$., and D. H. Fender (1972) The interaction of color and luminance in stereoscopic vision. Invcst. Ophthalmol. 11: 482-490.

Lund, J. S. (1973) Organization of neurons in the visual cortex, area 17, of the monkey (Macaca mulatta). J. Comp. Neurol. 147: 455475 .

Lund, J. S., and R. G. Boothe (1975) Interlaminar connections and pyramidal neuron organization in the visual cortex, area 17, of the macaque monkey. J. Comp. Neurol. 159: 305-334.

Lund, J. S., R. D. Lund, A. E. Hendrickson, A. H. Bunt, and A. F. Fuchs (1975) The origin of efferent pathways from the primary visual cortex, area 17, of the macaque monkey as shown by retrograde transport of horseradish peroxidase. J. Comp. Neurol. 164: 287-304.

MacKay, D. M. (1957) Moving visual images produced by regular stationary patterns. Nature $180: 849-850$

Malpeli, J. G., P. H. Schiller, and C. L. Colby (1981) Response properties of single cells in monkey striate cortex during reversible inactivation of individual lateral geniculate laminae. J. Neurophysiol. 46: 1102-1119.

Marc, R. E., and H. G. Sperling (1977) Chromatic organization of primate cones. Science 196: 454-456.

Marr, D., and T. Poggio (1976) Cooperative computation of stereo disparity. Science 194: 283-287.

Marr, D., and T. Poggio (1979) A computational theory of human stereo vision. Proc. R. Soc. Lond. [Biol.] 204: 301-328.

Maunsell, J. H. R., and D. C. Van Essen (1983a) Functional properties of neurons in middle temporal visual area of the macaque monkey. I. Selectivity for stimulus direction, speed, and orientation. J. Neurophysiol. 49: 1127-1147.

Maunsell, J. H. R., and D. C. Van Essen (1983b) Functional properties of neurons in middle temporal visual area of the macaque monkcy. II. Binocular interactions and sensitivity to binocular disparity. J Neurophysiol. 49: 1148-1167.

Mayhew, J. E. W., and S. M. Anstis (1972) Movement aftereffects contingent on color, intensity and pattern. Percept. Psychophys. 12: $77-85$.

Mayhew, J. E. W., and J. P. Frisby (1978) Stereopsis masking in humans is not orientationally tuned. Perception 7: 431-436.

McCollough, C. (1965) Color adaptation of edge-detectors in the human visual system. Science 149:1115-1116.

Merigan, W. H., and T. A. Eskin (1986) Spatio-temporal vision of macaques with severe loss of $\mathrm{P}_{3}$ retinal ganglion cells. Vision Res. 26: 1751-1761.

Mishkin, M., L. G. Ungerleider, and K. A. Macko (1983) Object vision and spatial vision: Two cortical pathways. Trends Neurosci. 6:414 417.

Morgan, M. J., and T. S. Aiba (1985) Positional acuity with chromatic stimuli. Vision Res. 25: 689-695.

Mullen, K. T. (1985) The contrast sensitivity of human colour vision to red-green and blue-yellow chromatic gratings. J. Physiol. (Lond.) 359: $381-400$

Müller-Lyer, F. C. (1894) Uber Kontrast und Konfluxion. Z. Psychol 9: 421.

Musatti, C. L. (1924) Sui fenomeni stereocinetici. Arch. It. Psicol. 3 : 105-120.

Navon, D. (1976) Irrelevance of figural identity for resolving ambiguities in apparent motion. J. Exp. Psychol. [Hum. Percept.] 2: 130138.

Naylor, E. J., and A. Stanworth (1954) Retinal pigment and the Haidinger effect. J. Physiol. (Lond.) 124: 543-552.

Necker, L. A. (1832) Observations on some remarkable optical phænomena scen in Switzerland; and on an optical phænomenon which occurs on viewing a figure of a crystal or geometrical solid. 
London and Edinburgh Philosophical Magazine and Journal of Science $1: 329-337$.

Neisser, U. (1968) The processes of vision. Sci. Am. 219: 204-214.

Newcombe, F., and W. R. Russell (1969) Dissociated visual perceptual and spatial deficits in focal lesions of the right hemisphere. J. Neurol. Neurosurg. Psychiatry 32: 73-81.

Østerberg, G. (1935) Topography of the layer of rods and cones in the human retina. Acta Ophthalmol. (Copenhagen) (Suppl. 6); also Nyt Nordisk Forlag, p. 102, Arnole Busck, Copenhagen.

Perry, V. H., R. Oehler, and A. Cowey (1984) Retinal ganglion cells that project to the dorsal lateral geniculate nucleus in the macaque monkey. Neuroscience 12: 1101-1123.

Pettigrew, J. D., T. Nikara, and P. O. Bishop (1968) Binocular interaction on single units in cat striate cortex: Simultaneous stimulation by single moving slit with receptive fields in correspondence. Exp. Brain Res. 6: 391-410.

Poggio, G. F., and B. Fischer (1977) Binocular interaction and depth sensitivity in striate and prestriate cortex of behaving rhesus monkey. J. Neurophysiol. 40: 1392-1405.

Poggio, G. F., and T. Poggio (1984) The analysis of stereopsis. Annu. Rev. Neurosci. 7: 379-412.

Poggio, G. F., B. C. Motter, S. Squatrito, and Y. Trotter (1985) Responses of neurons in visual cortex ( $\mathrm{V} 1$ and $\mathrm{V} 2$ ) of the alert macaque to dynamic random-dot stereograms. Vision Res. 25: 397-406.

Pohl, W. (1973) Dissociation of spatial discrimination deficits following frontal and parietal lesions in monkeys. J. Comp. Physiol. Psychol. 82: 227-239.

Polyak, S. (1957) The Vertebrate Visual System, U. Chicago P., Chicago.

Ponzo, M. (1913) Rapports entre quelques illusions, visuelles de contraste angulaire et l'appreciation de grandeur des astres a l'horizon. Arch. It. Biol. 58: 327-329.

Pulfrich, C. (1922) Die Stereoskopie im Dienste der isochromen und heterochromen Photometric. Naturwissenschaften 10:533-564, 569$601,714-722,735-743,751-761$.

Ramachandran, V.S. (1985) Apparent motion of subjective surfaces. Perception 14: 127-134.

Ramachandran, V.S., and S. M. Anstis (1983) Perceptual organization in moving patterns. Nature 304: 529-531.

Ramachandran, V.S., and S. M. Anstis (1985) Perceptual organization in multistable apparent motion. Perception 14: 135-143.

Ramachandran, V. S., and P. Cavanagh (1985) Subjective contours capture stereopsis. Nature 317: 527-530.

Ramachandran, V.S., and R. L. Gregory (1978) Does colour provide an input to human motion perception? Nature 275: 55-56.

Ramachandran, V. S., and S. Sriram (1972) Stereopsis generated with Julesz patterns in spite of rivalry imposed by colour filters. Nature 237: 347-348.

Ramachandran, V. S., A. Cronin-Golomb, and J. J. Myers (1986) Perception of apparent motion by commissurotomy patients. Nature 320: 358-359.

Riggs, L. A., F. Ratliff, J. C. Cornsweet, and T. N. Cornsweet (1953) The disappearance of steadily fixated visual test objects. J. Opt. Soc. Am. 43: 495-501.

Riley, B. (1964) "Current" (polymer paint on board). Collection Museum of Modern Art New York, Phillip Johnson Fund.

Rizzo, M., and H. Damasio (1985) Impairment of stereopsis with focal brain lesions. Ann. Neurol. 18: P112.

Rollmann, W. (1853) Notiz zur Stereoskopie. Pogg. Ann. 89: 350351

Rubin, E. (1915) Synsoplevede Figurer. Glydendalska, Copenhagen.

Rushton, W. A. H. (1965) Visual adaptation. (Ferrier Lecture, 1962.) Proc. Roy. Soc. Lond. [Biol.] 162: 20-46.

Schiller, P. H., and C. L. Colby (1983) The responses of single cells in the lateral geniculate nucleus of the rhesus monkey to color and luminance contrast. Vision Res. 23: 1631-1641.

Schiller, P. H., and J. G. Malpeli (1978) Functional specificity of lateral geniculate nucleus laminae of the rhesus monkey. J. Neurophysiol. 41: 788-797.

Schumann, F. (1900) Beitrage zur Analyse der Gesichtswakrnehmungen. Z. Psychol. 23: 1-32.

Shapley, R., and V. H. Perry (1986) Cat and monkey retinal ganglion cells and their visual functional roles. Trends Neurosci. 9: 229-235.

Shapley, R., E. Kaplan, and R. Soodak (1981) Spatial summation and contrast sensitivity of $X$ and $Y$ cells in the lateral geniculate nucleus of the macaque. Nature 292: 543-545.
Sherman, S. M. (1985) In Progress in Psychobiology and Physiological Psychology, vol. II, J. M. Sprague and A. N. Epstein, eds., pp. 233324, Academic, New York.

Shimojo, S. J., B. Bauer, Jr., K. M. O'Connell, and R. Held (1986) Pre-stereoscopic vision in infants. Vision Res. 26: 501-510.

Shipp, S., and S. Zeki (1985) Segregation of pathways leading from area V2 to areas V4 and V5 of macaque monkey visual cortex. Nature 315: 322-325.

Spatz, W. B. (1975) An efferent connection of the solitary cells of Meynert. A study with horseradish peroxidase in the marmoset $\mathrm{Cal}$ lithrix. Brain Res. 92: 450-455.

Stabell, U., and B. Stabell (1980) Variation in density of macular pigmentation and in short-wave cone sensitivity with eccentricity. $\mathrm{J}$. Opt. Soc. Am. 70: 706-711.

Steinhaus, H. (1938) Mathematical Snapshots, 1st Ed., Stechert, New York.

Steinman, R. M., R. J. Cunitz, G. T. Timberlake, and M. Herman (1967) Voluntary control of microsaccades during maintained monocular fixation. Science 155: 1577-1579.

Stockman, A., and D. I. A. MacLeod (1986) Visible beats from invisible flickering lights: Evidence that blue-sensitive cones respond to rapid flicker. Invest. Ophthalmol. Vis. Sci. (Suppl.) 27: 71.

Stromeyer, C. F., and B. M. Dawson (1978) Form-colour aftereffects: Selectivity to local luminance contrast. Perception 7: 407-415.

Stromeyer, C. F., and R. J. W. Mansfield (1970) Colored aftereffects produced with moving edges. Percept. Psychophys. 7: 108-114.

Tansley, B. W., and R. M. Boynton (1978) Chromatic border perception: The role of red- and green-sensitive cones. Vision Res. 18: 683697

Tansley, B. W., A. W. Robertson, and K. E. Maughan (1983) Chromatic and achromatic border perception: A two-cone model accounts for suprathreshold border distinctness judgments and cortical patternevoked response amplitudes to the same stimuli. In Colour Vision: Physiology and Psychophysics, J. Mollon and R. T. Sharpe, eds., pp. 445-454, Academic, New York.

Ihompson, P. (1982) Perceived rate of movement depends on contrast. Vision Res. 22: 377-380.

Tolhurst, D. J. (1973) Separate channels for the analysis of the shape and the movement of a moving visual stimulus. J. Physiol. (Lond.) 231: 385-402.

Tootell, R. B. H., M. S. Silverman, E. Switkes, and R. L. De Valois (1982) Deoxyglucose analysis of retinotopic organization in primate striate cortex. Science 218: 902-904.

Tootell, R. B. H., S. L. Hamilton, E. Switkes, and R. L. De Valois (1985) DG as a "functional HRP" in macaque striate cortex. Invest. Ophthalmol. Vis. Sci. (Suppl.) 26: 8.

Troxler, D. (1804) Ophthalmol. Bibliotek, vol. II, von Himley and Schmidt, eds., p. 1, Jena.

Tyler, C. W. (1975) Stereoscopic tilt and size aftereffects. Perception 4: $187-192$.

Ungerleider, L. G., T. W. Galkin, and M. Mishkin (1983) Visuotopic organization of projections from striate cortex to inferior and lateral pulvinar in rhesus monkey. J. Comp. Neurol. 217: 137-157.

Van Essen, D. C., and J. H. R. Maunsell (1983) Hierarchical organization and functional streams in the visual cortex. Trends Neurosci. 6: $370-375$.

Van Nes, F. L., J. J. Koenderick, H. Nas, and M. A. Bouman (1967) Spatiotemporal modulation transfer in the human eye. J. Opt. Soc. Am. 57: 1082-1088.

van Tuijl, H. F. J. M. (1975) A new visual illusion: Neonlike color spreading and complementary color induction between subjective contours. Acta Psychol. 39: 441-445.

van Tuijl, H. F. J. M., and C. M. M. de Weert (1979) Sensory conditions for the occurrence of the neon spreading illusion. Perception 8: 211-215.

Viénot, F. (1983) Can variation in macular pigment account for the variation of colour matches with retinal position? In Colour Vision: Physiology and Psychophysics, J. Mollon and R. T. Sharpe, eds., pp. 107-116, Academic, London.

von Bezold, W. (1876) The Theory of Color (transl. from German by S. R. Koehler), L. Prang, Boston.

Wald, G. (1967) Blue-blindness in the normal fovea. J. Opt. Soc. Am. 57: 1289-1301.

Wallace, G. K. (1966) Optical illusions. Nature 209: 327-328.

Wertheimer, M. (1912) Experimentelle Studien über das Sehen von Bewegung. Z. Psychol. 61: 161-265. 
Wertheimer, M. (1923) Untersuchungen zür Lehre von der Gestalt. II. Psychol. Forsch. 4: 301-350.

Westheimer, G. (1979) The spatial sense of the eye. Invest. Ophthalmol. Vis. Sci. 18: 893-912.

Westheimer, G. (1981) Visual hypcracuity. Prog. Sensory Physiol. 1: $1-30$.

Westheimer, G., and S. P. McKee (1979) What prior uniocular processing is necessary for stereopsis? Invest. Ophthalmol. Vis. Sci. 18: 614-621.

Wheatstone, C. (1838) On some remarkable, and hitherto unobserved, phenomena of binocular vision. Phil. Trans. R. Soc. Lond. [Biol.] 128: 371-394.

Wiesel, T. N., and D. H. Hubel (1966) Spatial and chromatic interactions in the lateral geniculate body of the rhesus monkey. J. Neurophysiol. 29: 1115-1156.

Williams, D. R., D. I. A. MacLeod, and M. M. Hayhoe (1981) Foveal tritanopia. Vision Res. 21: 1341-1356.

Wolfe, J. M., and D. A. Owens (1981) Is accommodation colorblind? Focusing chromatic contours. Perception 10: 53-62.
Yarbus, A. L. (1967) Eye Movements and Vision (trans. from Russian by B. Haigh; for American edition, L. A. Riggs), Plenum, New York. Zeki, S. M. (1969) Representation of central visual fields in prestriate cortex of monkeys. Brain Res. 14: 271-291.

Zeki, S. M. (1973) Cells responding to changing image size and disparity in the cortex of the rhesus monkey. J. Physiol. (Lond.) 242. $827-841$

Zeki, S. M. (1980a) The response properties of cells in the middle temporal area (area MT) of owl monkey visual cortex. Proc. R. Soc. Lond. [Biol.] 207: 239-248.

Zeki, S. M. (1980b) The representation of colours in the cerebral cortex. Nature 284: 412-418.

Zeki, S. M. (1983) The distribution of wavelength and orientation selectivity in different areas of monkey visual cortex. Proc. R. Soc. Lond. [Biol.] 207: 239-248.

Zöllner, F. (1860) Uber eine neue Art von Pseudoskopie. Pogg. Ann. 110: 500-525; Cosmos 18: 289-290; Z. Naturwissenschaft. 16: 6063. 